\title{
Comparing progression molecular mechanisms between lung adenocarcinoma and lung squamous cell carcinoma based on genetic and epigenetic networks: big data mining and genome- wide systems identification
}

\author{
Shan-Ju Yeh ${ }^{1}$, Chien-An Chang ${ }^{1}$, Cheng-Wei Li ${ }^{1}$, Lily Hui-Ching Wang ${ }^{2}$ and Bor-Sen \\ Chen ${ }^{1,3}$ \\ ${ }^{1}$ Laboratory of Automatic Control, Signaling Processing, and Systems Biology, Department of Electrical Engineering, National \\ Tsing Hua University, Hsinchu 30013, Taiwan \\ ${ }^{2}$ Department of Medical Science, Institute of Molecular and Cellular Biology, National Tsing Hua University, Hsinchu 30013, \\ Taiwan \\ ${ }^{3}$ Department of Electrical Engineering, Yuan Ze University, Chungli 32003, Taiwan \\ Correspondence to: Bor-Sen Chen, email: bschen@ee.nthu.edu.tw \\ Keywords: lung adenocarcinoma, lung squamous cell carcinoma, NSCLC, genetic and epigenetic network, potential drug target \\ Received: February 26, $2019 \quad$ Accepted: April 29, $2019 \quad$ Published: June 04, 2019 \\ Copyright: Yeh et al. This is an open-access article distributed under the terms of the Creative Commons Attribution License 3.0 \\ (CC BY 3.0), which permits unrestricted use, distribution, and reproduction in any medium, provided the original author and source \\ are credited.
}

\section{ABSTRACT}

Non-small-cell lung cancer (NSCLC) is the predominant type of lung cancer in the world. Lung adenocarcinoma (LADC) and lung squamous cell carcinoma (LSCC) are subtypes of NSCLC. We usually regard them as different disease due to their unique molecular characteristics, distinct cells of origin and dissimilar clinical response. However, the differences of genetic and epigenetic progression mechanism between LADC and LSCC are complicated to analyze. Therefore, we applied systems biology approaches and big databases mining to construct genetic and epigenetic networks (GENs) with next-generation sequencing data of LADC and LSCC. In order to obtain the real GENs, system identification and system order detection are conducted on gene regulatory networks (GRNs) and protein-protein interaction networks (PPINs) for each stage of LADC and LSCC. The core GENs were extracted via principal network projection (PNP). Based on the ranking of projection values, we got the core pathways in respect of KEGG pathway. Compared with the core pathways, we found significant differences between microenvironments, dysregulations of miRNAs, epigenetic modifications on certain signaling transduction proteins and target genes in each stage of LADC and LSCC. Finally, we proposed six genetic and epigenetic multiple-molecule drugs to target essential biomarkers in each progression stage of LADC and LSCC, respectively.

\section{INTRODUCTION}

Lung cancer is the most common malignancy resulting in the largest number of cancer-related deaths worldwide $[1,2]$. Only $15.9 \%$ of lung cancer patients survive more than 5 years [3]. Lung cancers are classified by histological types which have something to do with important implications for the clinical management and prognosis of the disease. There are two main histological groups of lung cancer which are non-small-cell lung cancer (NSCLC) and small-cell lung cancer. About 85\% of human lung cancers are non-small cell lung cancer [4]. NSCLCs are subdivided broadly into three major histological subtypes: lung adenocarcinoma (LADC), lung squamous cell carcinoma (LSCC), also called epidermoid carcinoma, and lung large-cell carcinoma (LLCC). Both LADC and LSCC are the predominant form of lung cancer accounting for the $40 \%$ and $33 \%$ majority of cancer deaths worldwide, respectively $[5,6]$.

Based on histopathological features and gene expression signatures, LADC and LSCC are widely thought to be characterized by a different cell of origin and 
a distinct pattern of molecular alterations. In general, for LADCs, which are thought to origin from the bronchiolar or alveolar epithelium (Clara cells or type II pneumocytes), mainly arise from the peripherally located airways and can be characterized by the production of mucin and/or the occurrence of glandular differentiation. For LSCCs, which typically often origin from the bronchial epithelium of larger and more central airways (basal cells), mostly arise from central lung and can be characterized by the morphological features of squamous differentiation, including squamous pearl formation, individual cell keratinisation, and intercellular bridges [5, 7-9].

One major risk factor of these two histologies is tobacco smoke and the other risk factors are asbestos, radon, and environmental/occupational exposure to polycyclic aromatic hydrocarbons and other pollutants contributing to the development of lung cancer, including LADC and LSCC [10, 11]. It has been reported that LADCs often occur in women and people who don't have smoke history, while LSCCs are highly correlated with exposure to tobacco smoke $[5,8,12]$. Though some molecular attributes are shared in these two subtypes, they represent unique molecular characteristics, distinct cells of origin, and different clinical responses [10]. Hence, LADC and LSCC could be seen as distinct diseases. However, the molecular mechanisms underlying carcinogenesis and tumor development of LADC and LSCC are not fully elucidated. Therefore, it is important to understand the carcinogenic mechanism of LADC and LSCC, which could provide more efficacious therapeutic strategies.

Recent studies indicate that carcinogenesis and cancer development not only be accounted for genetic alterations alone, but also involve epigenetic changes. Both genetic alterations and epigenetic changes could lead to the dysregulation of key tumor suppressor genes, oncogenes, and DNA repair/housekeeping genes. Despite the well-known role of genetic alterations, including copy number alterations and mutations in oncogenesis, it has been reported that epigenetic changes are much more frequent than genetic alterations in lung cancer $[10,13]$. The epigenetic changes include DNA methylation, histone modifications, and non-coding RNA, specific microRNA and lncRNA [10, 14]. Histone modifications and DNA methylation, which are important process for regulating gene transcription, play important roles in maintaining the normal development of cells. However, the anomalies in DNA methylation and histone modifications are associated with the carcinogenesis and development of tumor cells. It has been observed that DNA methylation was associated with tissue specificity and distinct DNA methylation patterns for LADC and LSCC have been identified [15-17].

MicroRNAs (miRNAs) are short non-coding RNAs with 19-25 nucleotides (nts) in length. They mediate posttranscriptional control of gene expression by incorporating with the RNA-induced silencing complex (RISC) and partially or perfectly bind to the $3^{\prime}$ untranslated regions
(3'-UTR) of target mRNA leading to either degradation of their target mRNAs or translational repression [18]. Experimental and bioinformatic studies have shown that more than $30 \%$ of human genes are direct targets of miRNA, implying that miRNAs are involved in the regulation of various biological processes including cell cycle regulation, cell growth, apoptosis, cell differentiation and stress reactions [14]. Previous study proposed a framework utilizing functional annotation information to identify coregulation between transcriptional and posttranscriptional layers [19]. Calin et al. have demonstrated that the locations of miRNA genes are frequently found at fragile sites in the genome, suggesting that the aberrant expression of miRNAs is associated with tumorigenesis [20]. Indeed, accumulating evidences show that the dysregulations of miRNAs are observed in human cancers, including NSCLC (LADC and LSCC), and may serve as oncogenes or tumor suppressors [21, 22].

In addition to miRNAs, long non-coding RNAs (lncRNAs) also have been caught the attention in recent year due to its role in the regulation of cancer development. LncRNA, non-protein-coding transcript that is longer than 200 nucleotides, can serve as regulators of gene expression and chromatin structure [23]. Numerous evidences showed that the expression of lncRNAs could alter various types of human cancers, including NSCLC (LADC and LSCC). It implies that the differential expressions of lncRNAs are associated with cancer pathogenesis and function as new regulators in cancer development [24, 25]. Moreover, it has been found that the binding affinities of transcription factors (TFs), RNA polymerase, miRNAs, and IncRNAs could be affected by DNA methylation $[26,27]$. Therefore, analyzing the regulation of miRNAs and lncRNAs in progression molecular mechanisms of LADC and LSCC is essential as well.

In recent year, the protein-protein interaction networks (PPINs) and gene regulatory network (GRNs) have been used to investigate the molecular mechanisms and found target genes based biomarkers [28, 29]. Focusing on global human gene regulatory network comprising both transcriptional and post-transcriptional regulatory relationships, and integrated the protein interactome, the topological properties for regulatory motifs has been investigated to reveal target crucial proteins [30]. Moreover, there is a study proposing computational method to predict lncRNA functions by identifying lncRNA-associated modules in protein-protein interaction network [31]. Gene expression profiles in different tissues could be used to discover disease-specific biomarker genes by evaluating Pearson correlation coefficient and Kolmogorov-Smirnov distance [32]. To date, many studies used gene and protein expression profiles with computational methods to identify new drug targets and biomarkers for drug discovery [33, 34]. Based on the examination of genes for tissue- and genespecific correlations, a large-scale analysis of protein 
abundance and gene expression across a diverse set of human tissues was proposed [35]. However, seldom research would consider epigenetic modifications effects to panoramic view of an extensive network. Therefore, in this study, we put focus on the comparison of progression molecular mechanisms and how epigenetic regulations and modifications contribute to the development of LADC and LSCC, respectively.

In order to construct real GRNs and PPIs for real GENs in each stage of LADC and LSCC (normal stage, early stage, middle stage, and advanced stage), we have applied system modeling, system identification, system order detection scheme and big database mining approaches on their corresponding next-generation sequencing (NGS) data. Since the real GENs are still too complicated to analyze, the core networks should be extracted from the real GENs. Previous literature has shown that network connectivity was predictive of function because interactions often occurred among functionally related genes [36]. However, core network was constructed by identified network hubs and core elements not in hubs would be neglected [37]. Here, we can use the principal network projection (PNP) method on genome-wide data to avoid this problem to extract the principal structure of real GENs from the perspective of significant network linking energy. By comparing the core GENs between two connective stages (normal to early stage, early to middle stage, middle to advanced stage) of LADC and LSCC, we respectively obtained differential core signaling pathways of LADC and LSCC to get an insight into the differential genetic and epigenetic progression mechanisms. Based on the analysis of core signaling pathways in each stage of LADC and LSCC, we found different microenvironment changes, dysregulation of miRNAs/lncRNAs, DNA methylation and epigenetic modifications would involve in the mechanisms of LADC and LSCC progression. Finally, we selected some significant network biomarkers as drug targets and proposed six genetic and epigenetic multiple-molecule drugs for early, middle, and advanced stage of LADC and LSCC to prevent disease progression, respectively.

\section{RESULTS}

In this study, a flowchart for constructing genomewide GENs, core GENs, and core signaling pathways of each progression stage of LADC and LSCC is shown in Figure 1. To further investigate the different genetic and epigenetic mechanisms between LADC and LSCC from normal stage to early stage, early stage to middle stage, and middle stage to advanced stage, we respectively constructed the genome-wide real GENs of each stage of LADC and LSCC by big databases mining, system modeling, and systematic analysis. Utilized the network visualizing software Cytoscape [38], the genome-wide real GENs of each stage of LADC and LSCC are shown in Supplementary Figures 1, 2, respectively. Besides, the numbers of identified nodes and edges of each stage of LADC and LSCC are shown in Supplementary Tables 1, 2 , respectively.

However, the genome-wide real GENs are still too complicated. Based on the PNP method, we considered the projection value (i.e., $D_{L}(k)$ and $D_{R}(t)$ at equation (38) in the Supplementary Materials) and extracted the core GENs shown in Supplementary Figures 3, 4 from the real GENs for each stage of LADC and LSCC, respectively. Here, we selected human proteins, which do not include TFs with the top 3000 projection values and human TFs with the top 300 projection values in all four stages of LADC and LSCC. Besides, miRNAs/lncRNAs, which connect to genes encoding those selected proteins and TFs, were also selected as core nodes of each stage. The core GENs of normal stage, early stage, middle stage, and advanced stage of LADC and LSCC are shown in Supplementary Figures 3, 4, respectively.

To further compared and investigated genetic and epigenetic progression mechanisms between LADC and LSCC from each progression stage, we constructed core signaling pathways in different stages of LADC and LSCC. Core signaling pathways start from core receptors passing through several core signal transduction proteins to the core TFs which would regulate corresponding core proteins, lncRNAs and miRNAs. The expression changes of these core elements are marked in the denotation of KEGG signal transduction pathways in each LADC and LSCC progression stage. Besides, we considered epigenetic modifications on proteins, such as ubiquitination, deubiquitination, acetylation, and deacetylation. These epigenetic modifications could be attributed to the changes of the basal level of $b_{j}$ between former stage and later stage in the stochastic protein interactive model of the PPIN in equation (1) (see Materials and Methods). With the basal level change between two connective stages over a PPI basal level threshold, the core proteins were postulated to be affected by some epigenetic modifications. In addition, in specific core signaling pathways, we only considered the epigenetic modification induced by different epigenetic proteins between two connective stages. If the expression of epigenetic protein has the lowest value within 4 stages of LADC and LSCC, the epigenetic protein will not be considered. Moreover, the genes with basal level changes which are higher than a threshold between two connective stages suggest that they are affected by DNA methylation. The core signaling pathways of each carcinogenic progression stage (normal stage to early stage, early stage to middle stage, and middle stage to advanced stage) are described in the followings and shown in Figures 2-4. 


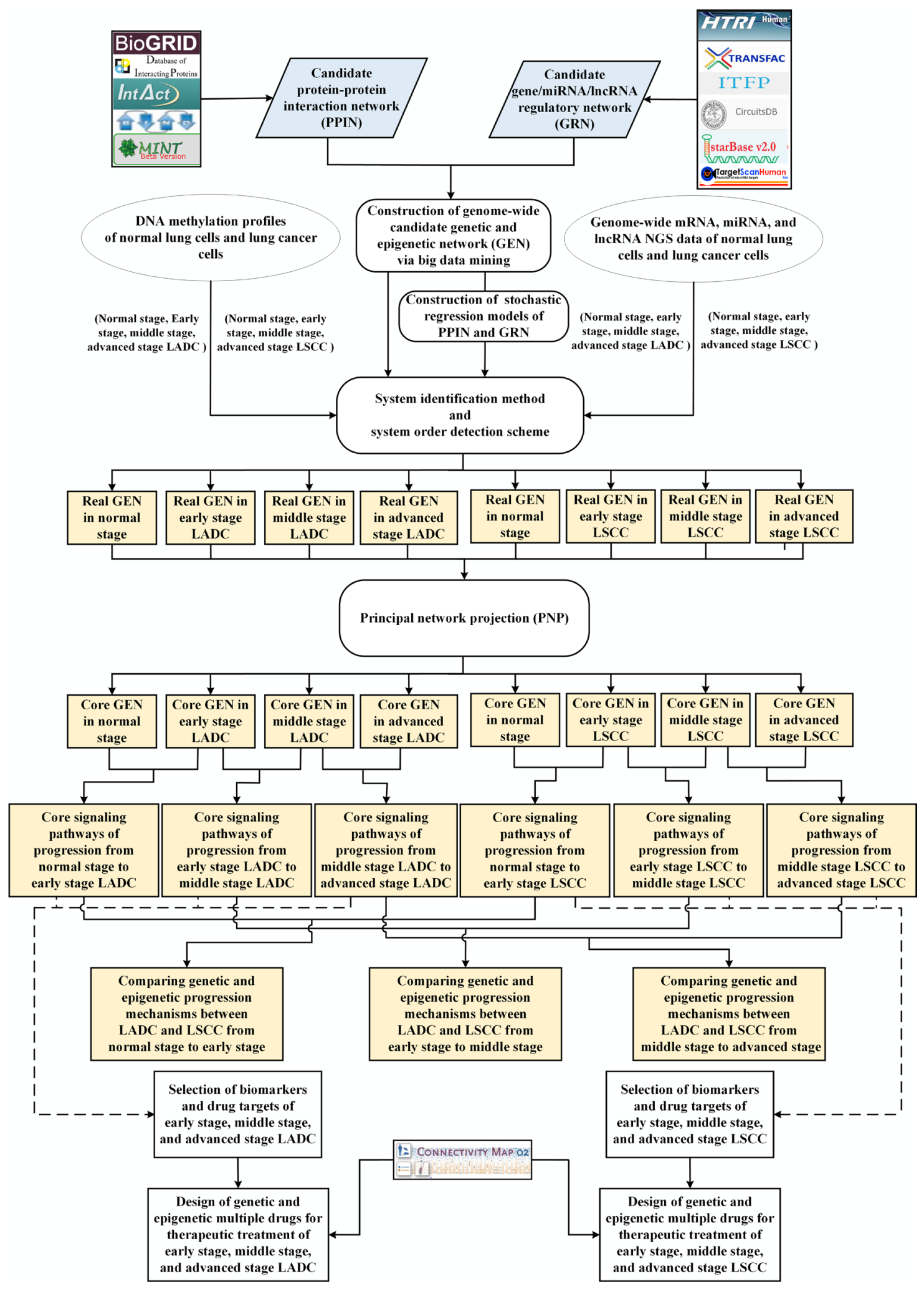

Figure 1: Flowchart of the construction for genome-wide GENS, core GENs, and core signaling pathways of each progression stage of LADC and LSCC and the discovery of potential genetic and epigenetic multiple drugs. The oval blocks represent the raw data of normal lung cells and lung cancer cells (i.e. early stage, middle stage, and advanced stage of LADC and LSCC), including genome-wide mRNA/miRNA/lncRNA NGS data and DNA methylation profiles. The blue grey blocks denote the candidate protein-protein interaction network (PPIN), which was constructed by databases (BIOGRID, IntAct, DIP, BIND, and MINT), and the candidate gene/miRNA/lncRNA regulatory network (GRN), which was constructed by databases (ITFP, HTRIdb, TRANSFAC, TargetScanHuman, CircuitDB, and StarBase2.0). The rounded rectangular blocks indicate the systems biology approach to be applied to 
construct genome-wide candidate GEN, real GENs of each stage (normal lung cells, early stage, middle stage, and advanced stage) of LADC and LSCC, and then to obtain core pathways of LADC and LSCC at each progression stage (normal cells to early stage, early stage to middle stage, and middle stage to advanced stage) by comparing the core GENs among the different stage. The light yellow blocks represent the identified information in our results, including real GENs at each stage and core signaling pathways of each progression stage. Besides, we selected biomarkers and drugtargets based on our results (dashed line with arrow) for designing genetic and epigenetic multiple drugs via CMap drug database mining for therapeutic treatment of early stage, middle stage, and advanced stage in LADC and LSCC, respectively.

\section{Analysis of core pathways to investigate different genetic and epigenetic progression mechanisms of LADC and LSCC from normal stage to early stage}

As shown in Figure 2, in normal stage of lung cells adjacent to the LADC, the receptors TLR4 and RET both interact with pro-inflammatory factor S100A9 and are activated by Lipopolysaccharide (LPS) and chemokine CCL2 to trigger protein S100A9 to modulate TFs, E2F1 and Sp1. The TF E2F1 affected by HECW2-induced ubiquitination negatively regulates inflammationrelated gene $M Y C$ and cell cycle-related gene $R B 1$. TF Sp1 negatively regulates telomere-related gene TERT and regulates gene CXCL5, involved in immune and inflammatory response. After receiving signaling from pro-inflammatory factor S100A10, the receptor EGFR then triggers the TF MYC mediated by cell cycle-related protein SEPT3, which belongs to the septin family of GTPases. The TF MYC then negatively regulates telomere-related gene TERT and immune-related genes $T G F B 1$.

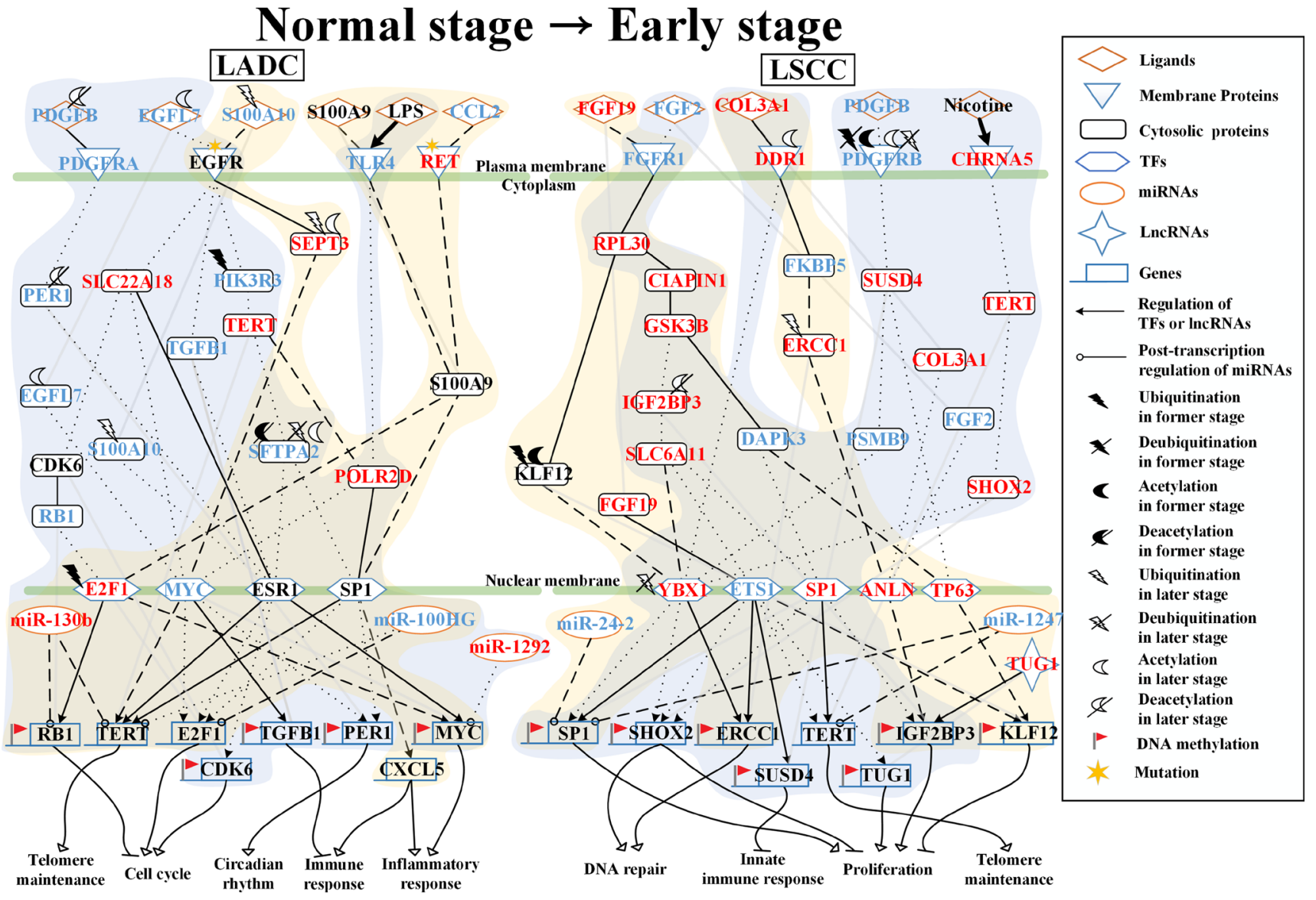

Figure 2: Core signaling pathways extracted from comparing genetic and epigenetic networks (GENs) between normal lung cells and early stage LADC and LSCC. The dot and dashed line represent the identified signaling pathways in former stage (normal stage) and later stage (early stage), respectively. Solid line indicates the common signaling pathways identified in both former stage and later stage. The yellow and blue regions are former stage (normal stage) and later stage (early stage), respectively. The lines without arrow denote the protein-protein interactions (PPIs). The lines with arrow represent the regulations of TFs and IncRNAs with activation and inhibition. The lines with circle are post-transcription regulations of miRNA with inhibition. Besides, the bold lines with arrow indicate the interaction or stimulation of proteins and xenobiotics. The Red font represent the node with significant differential expression change with a higher expression in later stage LADC and LSCC. While the blue font represent the node with a significant differential expression change with a lower expression in later stage LADC and LSCC. Besides, the gene with flag represents that this gene has basal level change between former and later stage LADC and LSCC, suggesting that the gene may be affected by DNA methylation. 
In early stage LADC, after receiving the signaling from PDGFB affected by HDAC4-induced deacetylation, the receptor PDGFRA triggers TF ESR1 mediated by protein PER1 affected by HDAC11-induced deacetylation. The TF ER $\alpha$ then negatively regulates cell cycle-related genes, $E 2 F 1$ and $C D K 6$. In addition, through interacting with EGFL7 affected by ELP3-induced acetylation, receptor EGFR then triggers the TFs, E2F1, c-Myc, ER $\alpha$, and Sp1, mediated by proteins, SLC22A18, CDK6, RB1, TGFB1, PIK3R3, TERT, and SFTPA2, which is affected by USP19-induced deubiquitination and KAT5-induced acetylation. The TF E2F1 negatively regulates cell cyclerelated gene $R B 1$ and the TF MYC negatively regulates cell cycle-related gene $E 2 F 1$ and circadian rhythm-related gene PER1. The TF ER $\alpha$ negatively regulates telomererelated gene TERT and positively regulates $M Y C$, and the TF $\mathrm{Sp} 1$ positively regulates the telomere-related gene TERT and negatively regulates cell cycle-related gene E2F1. Besides, the receptor TLR4 activated by LPS triggers TFs, c-Myc and Sp1. TF c-Myc positively regulates immune-related gene $T G F B 1$. TF Sp1 positively regulates circadian-related gene PER1. Moreover, the receptor TLR4 modulates TF MYC mediated by POLR2D to regulate immune-related gene $T G F B 1$.

Besides, in core signaling pathways between normal stage and early stage LADC, we also identified miRNAs, including miR-130b, miR-100HG, and miR1292 (Figure 2). MiR-130b inhibits RBI and TERT in normal stage and has a higher expression in early stage $\left(p\right.$-value $\left.<5.410^{-5}\right)$. MiR-100HG inhibits E2F1 in normal stage and TERT in early stage of LADC and has a higher expression in normal stage ( $p$-value $<1.1 \times 10^{-2}$ ). MiR-1292 inhibits MYC in the early stage of LADC and has a higher expression in the early stage of LADC

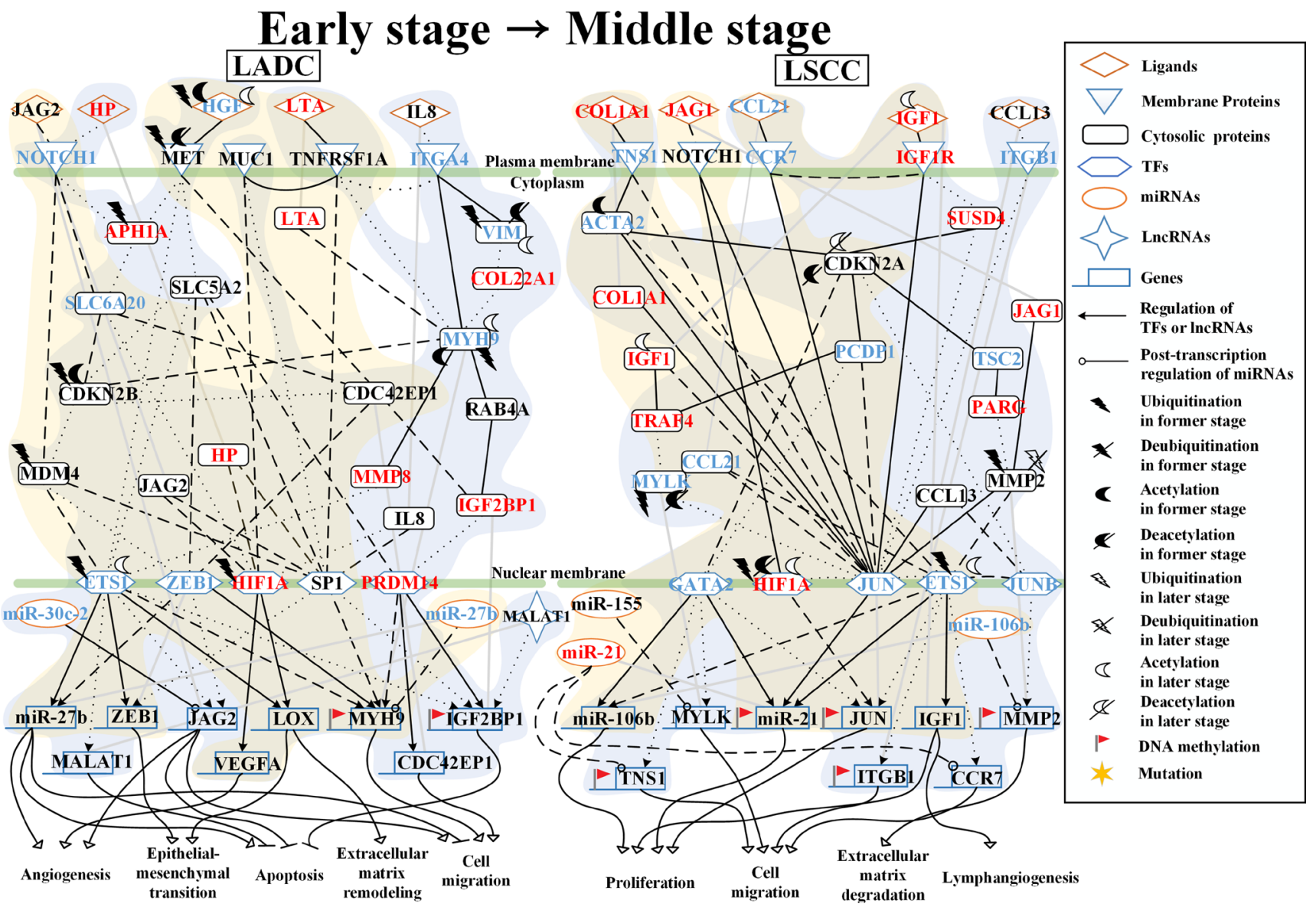

Figure 3: Core signaling pathways extracted from comparing genetic and epigenetic networks (GENs) between early stage and middle stage LADC and LSCC. The dot and dashed line represent the identified signaling pathways in former stage (early stage) and later stage (middle stage), respectively. Solid line indicates the common signaling pathways identified in both former stage and later stage. The yellow and blue regions are former stage (early stage) and later stage (middle stage), respectively. The lines without arrow denote the protein-protein interactions (PPIs). The lines with arrow represent the regulations of TFs and lncRNAs with activation and inhibition. The lines with circle are post-transcription regulations of miRNA with inhibition. The Red font represent the node with significant differential expression change with a higher expression in later stage LADC and LSCC. While the blue font represent the node with a significant differential expression change with a lower expression in later stage LADC and LSCC. Besides, the gene with flag represents that this gene has basal level change between former and later stage LADC and LSCC, suggesting that the gene may be affected by DNA methylation. 
( $p$-value $\left.<9.4 \times 10^{-6}\right)$. Moreover, there are five genes, $R B 1$, CDK6, TGFB1, PER1, MYC, with basal level difference than a threshold between normal stage and early stage LADC, suggesting that this might have been caused by DNA methylation on the corresponding genes. Our results indicate that in the normal stage of lung cells adjacent to the LADC, inflammatory response and immune response are caused by the mediation of genes, $M Y C$ and $C X C L 5$, and mediation of genes, TGFB 1 and $C X C L 5$, respectively. Telomere maintenance and cell cycle are inhibited by the mediation of gene TERT and mediation of genes, RBI and $M Y C$, respectively. However, in the early stage of LADC, telomere maintenance and cell cycle are caused by the mediation of gene TERT and mediation of genes, RBI, $E 2 F 1, C D K 6$, and $M Y C$, respectively. Immune response and circadian rhythm are inhibited by the mediation of gene TGFB1 and mediation of gene PER1, respectively.

In the normal stage of lung cells adjacent to the LSCC, the receptor FGFR1 is activated by fibroblast growth factor FGF19 to modulate TF YBX1 to positively regulate DNA repair-related gene $E R C C 1$ through proteins RPL30 and KLF12 affected by the UBE2J1-induced ubiquitination and KAT7-induced acetylation (Figure 2). Besides, receptor FGFR1 also modulates TF TP63 to positively regulate proliferation-related gene $K L F 12$. After binding to collagen COL3A1, receptor DDR1 then triggers the TF ANLN to negatively regulate proliferation-related gene IGF2BP3 by the mediation of proteins FKBP5 and ERCC1 affected by BTRC-induced ubiquitination in early stage LSCC.

In the early stage of LSCC, the receptor FGFR1 binds to FGF2 to modulate TFs ETS1 and Sp1 through the mediation of proteins RPL30, CIAPIN1, GSK3B, IGF2BP3, SLC6A11, and DAPK3. TF ETS1 then positively regulates proliferation-related gene $S P 1$ and negatively regulates DNA repair-related genes $E R C C 1$ and SHOX2, telomere-related gene TERT, proliferation-related gene TUG1, and gene SUSD4 involved in the innate

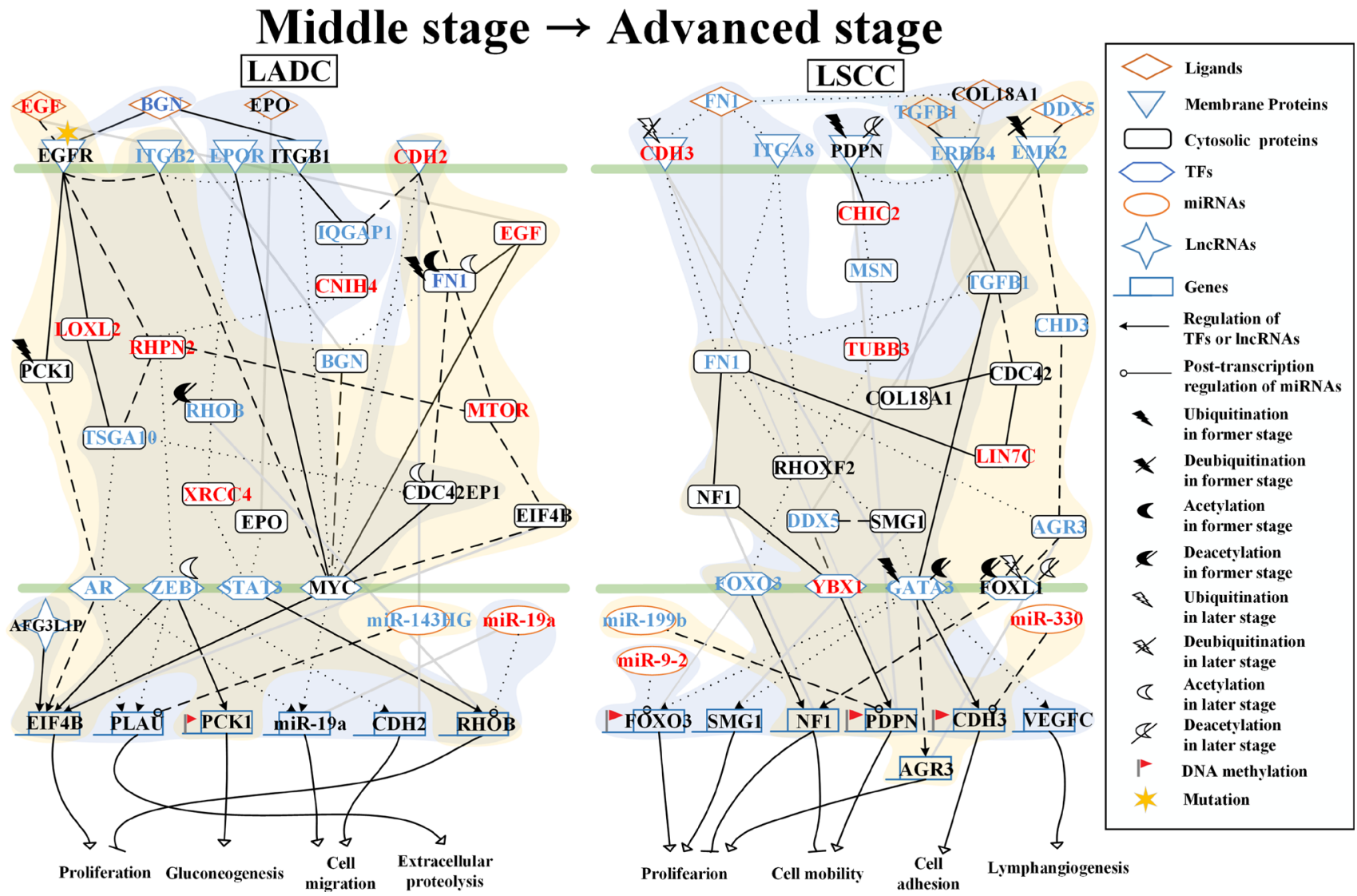

Figure 4: Core signaling pathways extracted from comparing genetic and epigenetic networks (GENs) between middle stage and advanced stage LADC and LSCC. The dot and dashed line represent the identified signaling pathways in former stage (middle stage) and later stage (advanced stage), respectively. Solid line indicates the common signaling pathways identified in both former stage and later stage. The yellow and blue regions are former stage (middle stage) and later stage (advanced stage), respectively. The lines without arrow denote the protein-protein interactions (PPIs). The lines with arrow represent the regulations of TFs and lncRNAs with activation and inhibition. The lines with circle are post-transcription regulations of miRNA with inhibition. The Red font represent the node with significant differential expression change with a higher expression in later stage LADC and LSCC. While the blue font represent the node with a significant differential expression change with a lower expression in later stage LADC and LSCC. Besides, the gene with flag represents that this gene has basal level change between former and later stage LADC and LSCC, suggesting that the gene may be affected by DNA methylation. 
immune response. The receptor DDR 1 , which is affected by SAT1-induced acetylation, interacts with collagen COL3A1 to directly trigger TF YBX1. Besides, PDGFRB and CHRNA5 are respectively activated by ligand PDGFB and xenobiotic nicotine to modulate TF YB-1 through proteins SUSD4 and PSMB9 and through proteins TERT and SHOX2, respectively. Then the TF YBX1 negatively regulates proliferation-related gene $\mathrm{Sp} 1$ and positively regulates DNA repair-related genes $S H O X 2$ and ERCC1.

Besides, in core signaling pathways between normal stage and early stage of LSCC, we identified miRNAs, including miR-24-2 and miR-1247, and lncRNA, TUG1 (Figure 2). MiR-24-2 inhibits SP1 in normal stage and has a higher expression in normal stage $(p$-value $<1.7 \times$ $\left.10^{-27}\right)$. MiR-1247 inhibits SPI and TERT in normal stage and has a higher expression in normal stage ( $p$-value $<$ $\left.6.7 \times 10^{-21}\right)$. TUG1 positively regulates gene $I G F 2 B P 3$ in the normal stage and has a higher expression in early stage of LSCC $\left(p\right.$-value $\left.<2.4 \times 10^{-5}\right)$. Moreover, there are seven genes, SP1, SHOX2, ERCC1, SUSD4, TUG1, $I G F 2 B P 3$, and $K L F 12$, with basal level difference which are than a threshold between normal stage and early stage LSCC, suggesting that this might have been caused by DNA methylation on the corresponding genes. Our results indicate that in the normal stage of lung cells which are adjacent to the LSCC, DNA repair is caused by the mediation of gene $E R C C 1$ and proliferation is inhibited by the mediation of genes, $K L F 12$ and $I G F 2 B P 3$. However, in the early stage of LSCC, telomere maintenance and proliferation are caused by the mediation of gene TERT and mediation of genes, $S P 1, S H O X 2, T U G 1$, and $I G F 2 B P 3$, respectively. DNA repair and innate immune response are inhibited by the mediation of genes, $\mathrm{SHOX} 2$ and $E R C C 1$, and mediation of gene $S U S D 4$, respectively.

\section{Analysis of core pathways to investigate different genetic and epigenetic progression mechanisms of LADC and LSCC from early stage to middle stage}

As shown in Figure 3, in the early stage of LADC, ligand JAG2 binds to receptor NOTCH1, the activated NOTCH1 then triggers the downstream proteins, MDM4, which is affected by UBE2U-induced ubiquitination, SLC6A20, and CDC42EP1 to modulate TFs, ETS1 and ZEB1. The TF ETS1 affected by NHLRC1-induced ubiquitination negatively regulates gene $m i R-27 b$, involved in angiogenesis, epithelial-mesenchymal transition (EMT) and cell migration, and positively regulates EMT-related genes, $Z E B 1$ and $L O X$, which is also involved in extracellular matrix (ECM) remodeling, and migration-related gene $M Y H 9$. Besides, the receptor MET, which is affected by MIB2-induced ubiquitination and HDAC9-induced deacetylation, interacts with its ligand HGF, which is affected by NEURL4-induced ubiquitination and NAT14-induced acetylation, to modulate TF ZEB1. TF ZEB1 then positively regulates genes, MYH9 and miR27b. Moreover, the ligand LTA binds to TNFRSFA, and TNFRSFA then directly triggers the TF Sp1 and indirectly triggers the TF HIF1A, which is affected by VHL-induced ubiquitination, through protein MUC1 to negatively regulate migration-related gene MYH9 and to positively regulate angiogenesis-related gene VEGFA and ECM remodeling-related gene $L O X$.

In the middle stage of LADC, after interacting with HP, NOTCH1 interacts with Gamma-Secretase subunit APH1A to trigger the downstream protein SLC6A20 to modulate TF HIF1A. TF HIF $1 \alpha$ then positively regulates angiogenesis-related gene $V E G F A$, ECM remodelingrelated and EMT-related gene $L O X$. After receiving IL8 signaling, ITGA4 then modulates TF ETS1, which is affected by ATAT1-induced acetylation, and TF PRDM14 through the mediation of proteins VIM, which is affected by KAT7-induced acetylation, COL22A1, and MYH9, which is affected by NAT6-induced acetylation. TF ETS1 affected by ATAT1-induced acetylation then negatively regulates migration-related gene $m i R-27 b$ and positively regulates EMT-related and ECM remodeling genes, $J A G 2, Z E B 1$, and $L O X$. TF PRDM14 positively regulates migration-related gene $C D C 42 E P 1$ and anti-apoptosisrelated gene $I G F 2 B P 1$. Besides, receptor MET is activated by binding to its ligand HGF to trigger TFs, ETS1, ZEB1, and HIF1A through the mediation by proteins, SLC5A2, CDKN2B, MDM4, APH1A, and SLC6A20. TF ETS1 negatively regulates anti-apoptosis-related genes IGF2BPI and MALAT1. TF ZEB1 negatively regulates migrationrelated genes $M Y H 9$ and $J A G 2$. TF HIF1A positively regulates angiogenesis-related genes $V E G F A$ and $L O X$, which are also involved in EMT and ECM remodeling. In addition, after ligand LTA binding to receptor TNFRSFA, the activated TNFRSFA triggers the TF Sp1 to positively regulate anti-apoptosis-related gene $I G F 2 B P 1$ and EMTrelated gene $Z E B 1$ through protein MUC1.

Besides, in core signaling pathways between early stage and middle stage of LADC, we identified miRNAs, including miR-30c2 and miR-27b, lncRNA, and MALAT1 (Figure 3). MiR-30c2 inhibits $J A G 2$ in both early stage and middle stage and has a higher expression in early stage compared to middle stage LADC ( $p$-value $<4.7$ $\left.\times 10^{-3}\right)$. MiR-27b inhibits MYH9 in early stage and has a higher expression in early stage compared to middle stage LADC ( $p$-value $<2.7 \times 10^{-2}$ ). MALAT1 negatively regulates gene $I G F 2 B P 1$ in middle stage and has a higher expression in early stage LADC ( $p$-value $<6.3 \times 10^{-3}$ ). Moreover, there are two genes, $M Y H 9$ and $I G F 2 B P 1$, with basal level differences which are higher than a threshold between early and middle stage of LADC, suggesting that this might have been caused by DNA methylation on the corresponding genes. Our results indicate that in the early stage of LADC, apoptosis is caused by the mediation of gene miR-27b. Angiogenesis, cell migration, ECM remodeling, and EMT are inhibited by the mediation of 
genes, $m i R-27 b$ and $V E G F A$, mediation of genes, $m i R$ 27b, MYH9, and CDC42EPI, mediation of gene $L O X$, and mediation of genes, ZEBI and $L O X$, respectively. However, in the middle stage of LADC, angiogenesis, cell migration, EMT, and ECM remodeling are caused by the mediation of genes, $m i R-27 b$ and $V E G F A$, mediation of genes, $J A G 2, m i R-27 b, M Y H 9$, and CDC42EP1, mediation of genes, ZEB1 and $L O X$, and mediation of gene $L O X$, respectively. Apoptosis is inhibited by mediation of genes, miR-27b, MALAT1, JAG2, and IGF2BP1.

In the early stage of LSCC, the tensin TNS1 interacts with collagen COL1A1 to trigger the TFs, ETS1, GATA2, and JUN through the mediation of proteins, ACTA2, which is affected by CREBBP-induced acetylation and CDKN2A, which is affected by HDAC4induced deacetylation (Figure 3). TF ETS1, which is affected by UBE2Q1-induced ubiquitination, negatively regulates proliferation-related genes $m i R-106 \mathrm{~b}$ and $m i R$ 21 and positively regulates proliferation-related gene IGF1. TF GATA2 positively regulates proliferationrelated genes $m i R-106 b$ and $m i R-21$. TF JUN positively regulates $m i R-21$. Besides, TF JUN is also modulated by receptors, NOTCH1 CCR7, and IGF1R, which are activated respectively by the binding of ligands, JAG1, CCL21, and IGF1, to positively regulate proliferation gene $m i R-21$. The activated NOTCH1 can also directly trigger TF HIF1A, which is affected by UBE2Z-induced ubiquitination and HDAC5-induced deacetylation, to negatively regulate proliferation-related gene JUN.

In the middle stage of LSCC, TNS1 interacts with COL1A1 to trigger TF GATA2 by the mediation of proteins, ACTA2, CDKN2A affected by HDAC1induced deacetylation, and PCDP1, to positively regulate cell migration-related genes, TNS1 and MYLK, and proliferation-related gene $m i R-21$, and to negatively regulate proliferation-related gene miR-106b. Through binding to ligand CCL21, receptor CCR7 triggers TF $\mathrm{JUN}$ to positively regulate proliferation-related gene $\mathrm{miR}$ 21. In addition, the activated IGF-1R binds to its ligand IGF-1, which is affected by EP300-induced acetylation to trigger the TFs, JUN, ETS1 affected by NAT9-induced acetylation, and JUNB, through the mediation of proteins, SUSD4, CDKN2A, PCDP1, TRAF4, MYLK, TSC2, PARG, and MMP2 in the signaling pathways. TF JunB positively regulates gene $M M P 2$ involved in extracellular matrix (ECM) degradation and both TFs, JUN and ETS1, which positively regulate proliferation-related genes, $m i R$ 21 and $I G F 1$, and to negatively regulate proliferationrelated gene $J U N$. Moreover, after receiving the signaling from chemokine CCL13, the activated ITGB1 triggers TFs, JUN, and ETS1 to positively regulate cell migrationrelated genes ITGBI and CCR7.

Besides, in core signaling pathways between early stage and middle stage of LSCC, we identified miRNAs, including miR-21, miR-155, and miR-106b (Figure 3). MiR-21 inhibits TNS1 and CCR7 in early stage and has higher expression in the middle stage when compared to the early stage of LSCC. MiR-155 inhibits MYLK in the early stage of LSCC and has higher expression in the early stage when compared to the middle stage of LSCC. MiR-106b inhibits MMP2 in the early stage of LSCC and has a higher expression in the early stage when compared to the middle stage of LSCC ( $p$-value $<$ $\left.8.1 \times 10^{-3}\right)$. Moreover, there are five genes, TNS1, miR21, JUN, ITGB1, MMP2, with basal level differences which are higher than a threshold between the early and middle stage of LSCC, suggesting that this might have been caused by DNA methylation on the corresponding genes. Our results indicate that in the early stage of LSCC, proliferation is caused by the mediation of genes, $m i R-106$, $I G F 1, m i R-21$, and JUN. However, in the middle stage of LSCC, proliferation, cell migration, ECM degradation, and lymphangiogenesis all are caused by the mediation of genes, $m i R-106 b, I G F 1, m i R-21$, and $J U N$, mediation of genes, TNS1, MYLK, ITGB1, and CCR7, mediation of gene $M M P 2$, and mediation of gene $I G F 1$, respectively.

\section{Analysis of core pathways to investigate different genetic and epigenetic progression mechanisms of LADC and LSCC from middle stage to advanced stage}

As shown in Figure 4, in the middle stage of LADC, receptor EGFR is activated by interacting with ligands EGF and BGN to trigger downstream protein PCK1, which is affected by UBE2D3-induced ubiquitination to modulate TF AR to positively regulate proliferationrelated gene EIF4B. Besides, the activated EGFR also modulates TF MYC to positively regulate $E I F 4 B$ through the mediation of proteins, RHPN2, MTOR, and EIF4B in the corresponding signaling pathways. The protein $\mathrm{CDH} 2$ can also modulate TF MYC through the mediation of proteins, FN1, MTOR, EIF4B, and CDC42EP1 in the corresponding signaling pathways to regulate proliferation-related gene $E I F 4 B$ positively.

In the advanced stage of LADC, receptor EGFR is activated by ligand BGN to modulate TF AR to negatively regulate gene $P L A U$, which is involved in extracellular proteolysis, and migration-related gene $m i R-19 a$ and to modulate TF MYC to positively regulate migration-related gene $m i R-19 a$ through the mediation of proteins LOXL2, TSGA10, and CDC42EP1 in the corresponding signaling pathways. After binding with the ligand EPO, the receptor EPOR then modulates the TF STAT3 to positively regulate proliferation-related gene $R H O B$ and also modulate TF MYC to positively regulate $m i R-19 a$ through the mediation of proteins, RHOB affected by SIRT1induced deacetylation in middle stage, and XRCC4 in the corresponding signaling pathways. Besides, ITGB1 is activated by interacting with ligand $\mathrm{BGN}$ to modulate $\mathrm{TF}$ ZEB1, which is affected by ESCO1-induced acetylation, to positively regulate gene $P L A U$, gluconeogenesis-related 
gene $P C K 1$, and migration-related gene $C D H 2$ through the mediation of proteins, IQGAP1, CNIH4, and RHPN2 in the corresponding signaling pathways. In addition, the activated ITGB1 and N-cadherin (CDH2) can both directly trigger TF MYC to regulate migration-related gene $m i R-19 a$ positively.

Besides, in core signaling pathways between the middle stage and advanced stage of LADC, we identified miRNAs, including miR-143HG and miR-19a, and lncRNA, AFG3L1P (Figure 4). MiR143HG inhibits PLAU in middle stage and has a higher expression comparing to the advanced stage of LADC. MiR-19a inhibits $R H O B$ in the advanced stage of LADC and has a higher expression comparing to the middle stage of LADC ( $p$-value $<3.4$ $\times 10^{-2}$ ). AFG3L1P negatively regulates EIF4B in both middle and advanced stage of LADC and has a higher expression in middle stage comparing to the advanced stage of LADC. Moreover, there is a gene PCK1, with basal level difference which is higher than a threshold between middle and advanced stage of LADC, suggesting that this might have been caused by DNA methylation on the corresponding gene. Our results indicate that in the middle stage of LADC, proliferation is caused by the mediation of gene EIF4B. However, in the advanced stage of LADC, proliferation is caused by the mediation of genes, EIF4B and RHOB; gluconeogenesis is caused by the mediation of gene PCK1; cell migration is caused by the mediation of genes, $m i R-19 a$ and $C D H 2$; extracellular proteolysis is caused by the mediation of gene PLAU.

In the middle stage of LSCC, the receptor EMR2, which is affected by the USP20-induced deubiquitination, is activated by interacting with DDX5 to modulate TF FOXL1, which is affected by the HDAC9-induced deacetylation to positively regulate gene NF1 involved in proliferation and cell mobility through the mediation of proteins $\mathrm{CHD} 3$ and AGR3 in the corresponding signaling pathways (Figure 4). After binding to ligand TGFB1, the receptor ERBB4 then modulates TF GATA3, which is affected by the SIAH1-induced ubiquitination and HDAC5-induced deacetylation, to positively regulate proliferation-related gene $A G R 3$ and gene $C D H 3$ involved in cell adhesion. Besides, the receptor ERBB4 can also modulate TF YBX1 to negatively regulate gene PDPN, which is involved in cell mobility through the mediation of proteins, CDC42, LIN7C, FN1, and NF1 in the corresponding signaling pathways.

In the advanced stage of LSCC, receptor ERBB4 activated by the binding to COL18A1, which interacts with FN1. Both receptor CDH3 affected by OTUD1-induced deubiquitination and the receptor ITGA8 have interactions with FN1 to modulate TFs, GATA3, YBX-1, and FOXO3 through the mediation of proteins, FN1, NF1, RHOXF2, and SMG1 in the corresponding signaling pathways. TF GATA3 then positively regulates proliferation-related gene $S M G 1$ and lymphangiogenesis-related gene $V E G F C$ and negatively regulates proliferation-related gene $\mathrm{FOXO} 3$ and gene $\mathrm{CDH} 3$ involved in cell adhesion. TF YBX1 negatively regulates gene $P D P N$, which is involved in cell mobility. TF FOXO3 positively regulates gene $N F 1$, which participates in proliferation and cell mobility. Moreover, the membrane glycoprotein PDPN, which is affected by SIRT1-induced deacetylation, can interact with ERBB4 to modulate TFs, FOXO3 and GATA3 to regulate genes, $N F 1$, FOXO3, SMG1, CDH3, and VEGFC, through the mediation of proteins, CHIC2, MSN, TUBB3, and RHOXF2 in the corresponding signaling pathway.

Besides, in core signaling pathways between the middle and advanced stage of LSCC, we identified miRNAs, including miR-199b, miR-330, and miR-9-2 (Figure 4). MiR-199b inhibits $P D P N$ in the middle stage and has a higher expression comparing to the advanced stage of LSCC. MiR-330 inhibits $\mathrm{CDH} 3$ in middle stage and has a higher expression in advanced stage comparing to the middle stage of LSCC ( $p$-value $<3.4 \times 10^{-2}$ ). MiR9-2 inhibits $\mathrm{FOXO} 3$ in advanced stage and has a higher expression comparing to the middle stage of LSCC. Moreover, there are three genes, FOXO3, PDPN, and $C D H 3$, with basal level differences which are higher than a threshold between middle stage and advanced stage LSCC, suggesting that this might have been caused by DNA methylation on the corresponding genes. Our results indicate that in the middle stage of LSCC, proliferation is caused by the mediation of genes, AGR3 and NF1. Cell adhesion and cell mobility are inhibited by the mediation of gene $C D H 3$ and mediation of genes, NF1 and $P D P N$, respectively. However, in the advanced stage of LSCC, the proliferation is caused by the mediation of genes, FOXO3, SMG1, and NF1; the cell mobility is caused by the mediation of genes, $N F 1$ and $P D P N$; the cell adhesion is caused by the mediation of gene $\mathrm{CDH}$; the lymphangiogenesis is caused by the mediation of gene VEGFC.

\section{Comparing and summarizing the differential genetic and epigenetic progression mechanisms caused by the core signaling pathways within connective stages of LADC and LSCC}

In Figure 5, we summarize the differential genetic and epigenetic progression mechanisms caused by the core signaling pathways in connective stages which are from normal to early stage, early to middle stage, and middle to advanced stage of LADC and LSCC. The carcinogenic progression from normal to early stage of LADC results from abnormal cellular functions, such as telomere maintenance, cell cycle, circadian rhythm, immune response, and inflammatory response. These are caused by dysregulation of signaling pathways (PDGFRA, EGFR, TLR4, and RET signaling pathways), dysregulation of ubiquitination and acetylation, DNA methylation on genes (RB1, CDK6, TGFB1, PER1, and MYC), and abnormal miRNAs silencing (miR-130b, miR-100HG, miR-1292). 


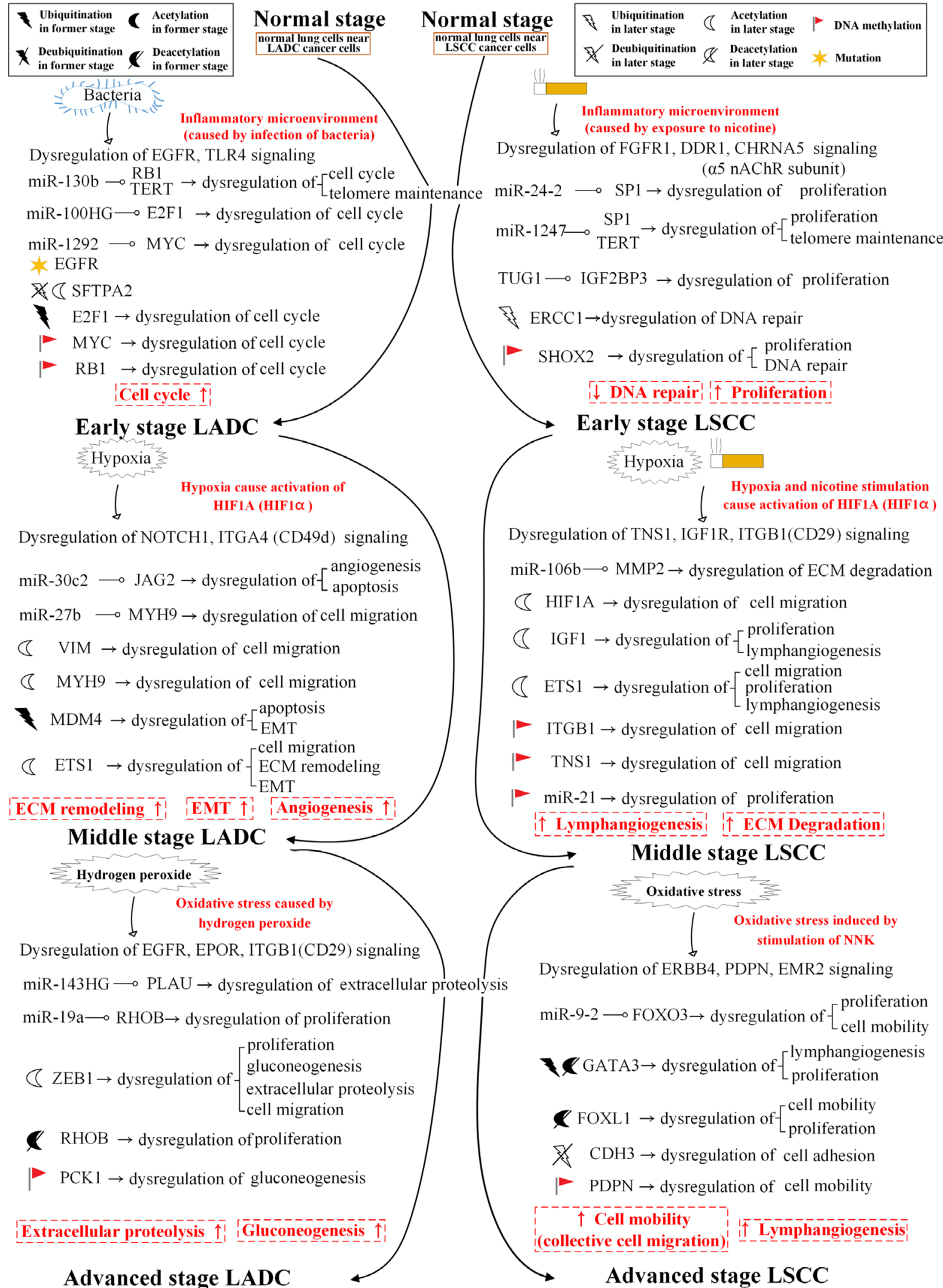

Figure 5: Summarizing the differential genetic and epigenetic progression mechanisms from normal stage to early stage, early stage to middle stage, and middle stage to advanced stage LADC and LSCC. The figure summarizes the differential genetic and epigenetic progression mechanisms caused by core signaling pathways within each connective stage of LADC and LSCC. The red font with dash-line rectangular blocks denote the differential function between LADC and LSCC. 
While the carcinogenic progression from normal to early stage of LSCC results from abnormal cellular functions, such as telomere maintenance, DNA repair, proliferation, and innate immune response. These bring about by dysregulation of signaling pathways (FGFR1, DDR1, PDGFRB, and $\alpha 5 \mathrm{nAChR}$ subunit signaling pathways), dysregulation of ubiquitination and acetylation, DNA methylation on genes (SP1, SHOX2, ERCC1, SUSD4, TUG1, IGF2BP3, and KLF12), abnormal miRNAs silencing (miR-24-2, miR-1247) and lncRNA regulation (TUG1).

The carcinogenic progression from early to middle stage of LADC gives rise to abnormal cellular functions, such as angiogenesis, EMT, apoptosis, ECM remodeling, and cell migration. These are caused by dysregulation of signaling pathways (NOTCH1, MET, TNFRSF1A, and ITGA4 signaling pathways), dysregulation of ubiquitination and acetylation, DNA methylation on genes ( $M Y H 9$ and IGF2BPI), abnormal miRNAs silencing (miR30c-2 and miR-27b) and lncRNA regulation (MALAT1). The carcinogenic progression from early to middle stage of LSCC results from abnormal cellular functions, such as proliferation, lymphangiogenesis, ECM degradation, and cell migration. These are caused by dysregulation of signaling pathways (TNS1, NOTCH1, CCR7, IGF1R, and ITGB1 signaling pathways), dysregulation of ubiquitination and acetylation, DNA methylation on genes (TNS1, miR-21, JUN, ITGB1 and MMP2), and abnormal miRNAs silencing (miR-21, miR-21, and miR-106b).

The carcinogenic progression from middle to advanced stage of LADC contributes to abnormal cellular functions, such as proliferation, gluconeogenesis, cell migration, and extracellular proteolysis. These are caused by dysregulation of signaling pathways (EGFR, EPOR, ITGB1 and $\mathrm{CDH} 2$ signaling pathways), dysregulation of ubiquitination and acetylation, DNA methylation on gene (PCK1), abnormal miRNAs silencing (miR-143HG and miR-19a) and IncRNA regulation (AFG3L1P). In contrast, the carcinogenic progression from middle to advanced stage of LSCC contributes to abnormal cellular functions, such as proliferation, lymphangiogenesis, cell mobility, and cell adhesion. These are due to dysregulation of signaling pathways (ITGA8, PDPN, ERBB4, EMR2, and $\mathrm{CDH} 3$ signaling pathways), dysregulation of ubiquitination and acetylation, DNA methylation on genes (FOXO3, PDPN, and $C D H 3$ ), and abnormal miRNAs silencing (miR-9-2, miR-199b, and miR-330).

To further investigate the impacts of miRNA regulations, DNA methylation, and epigenetic modifications on the genetic and epigenetic progression mechanisms between connective stages of LADC and LSCC, we extracted the specific core signaling pathways (Figure 6) out from these identified core signaling pathways (Figures 2-4) in each progression stage of LADC and LSCC. The more detailed comparison of genetic and epigenetic progression mechanisms will be discussed in the following sections.

\section{DISCUSSION}

\section{Microenvironment change, dysregulation of miRNA/IncRNA regulation, DNA methylation, and epigenetic modification contribute to different genetic and epigenetic mechanisms from normal stage progresses to early stage of LADC and LSCC}

As shown in Figure 6A and 6B, we identified that the inflammatory microenvironmental factor induced by bacteria infection (LPS) can potentially cause normal cells to progress to the early stage of LADC. Similarly, the inflammatory microenvironment induced by exposure to xenobiotic toxicity nicotine can potentially cause normal cells to progress to the early stage of LSCC. In general, LADC, which is thought to origin from the bronchiolar or alveolar epithelium (Clara cells or type II pneumocytes), mainly arises from the peripherally located airways, whereas LSCC, which typically often originates from the bronchial epithelium of larger and more central airways (basal cells), mostly arises from central lung $[5,7-9,39]$. Due to the different microenvironments, normal cells, which were nearby LADC and LSCC, receive different altered external signaling stimulations from their environment, and thus allow normal lung cells to have the potential to progress to LADC and LSCC through different genetic and epigenetic mechanisms, respectively. In LADC, inflammatory microenvironment induced by bacteria infection (LPS) can potentially cause the dysregulation of EGFR and TLR4 signaling pathways. Besides, the mutation of receptor EGFR, ubiquitination of E2F1, deubiquitination and acetylation of SFTPA2, dysregulation of DNA methylation in $R B 1$ and $M Y C$, and dysregulation of miR-130b, miR-100HG, and miR-1292 could also result in abnormal cellular functions, such as cell cycle, telomere maintenance, and inflammatory response, causing normal cells to progress to early stage of LADC. In LSCC, microenvironment exposed to xenobiotic toxicity nicotine can potentially cause the dysregulation of FGFR1, DDR1, and CHRNA5 ( $25 \mathrm{nAChR}$ subunit) signaling pathways. Moreover, the ubiquitination of ERCC1, dysregulation of DNA methylation in SHOX2 and TUG1, dysregulation of miR24-2 and miR-1247, and dysregulation of IncRNA TUG1 result in abnormal cellular functions such as DNA repair, proliferation, and telomere maintenance, causing normal cells to progress to early stage of LSCC.

Interestingly, the epidemiologic and clinical studies have indicated the strong relation between inflammation, chronic infection, and cancer [40-42]. Besides, it is not surprising that microbiome, including bacteria, can be found in lung since the inhalation of air is one of sources 

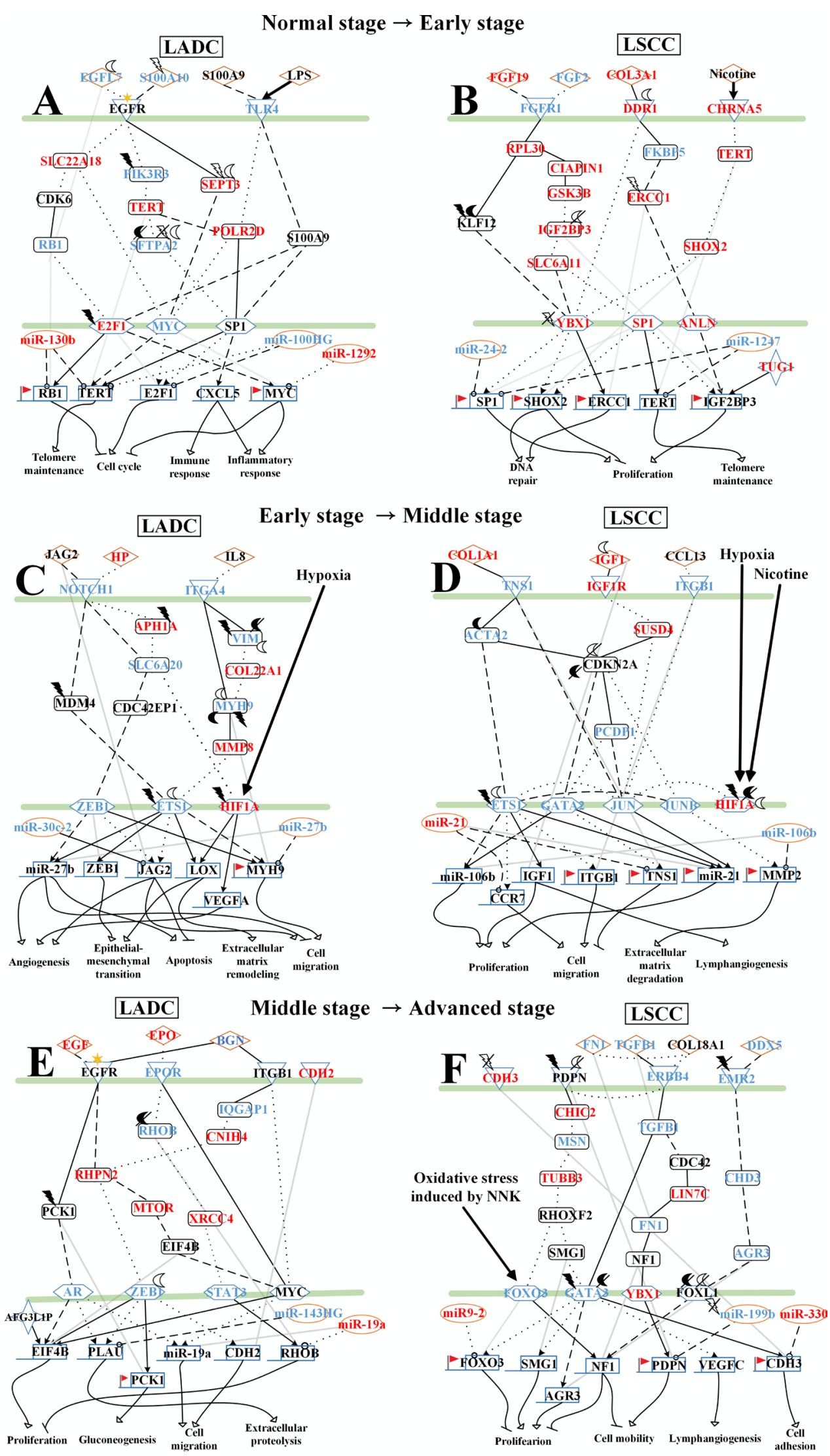

Figure 6: The specific core signaling pathways extracted from Figures $2-\mathbf{4}$ for investigating the differential progression molecular mechanisms between LADC and LSCC. (A) The genetic and epigenetic progression mechanisms from normal stage to early stage LADC could be potentially caused by inflammatory microenvironment induced by bacteria infection (LPS), dysfunctions of 
EGFR, and TLR4 signaling, regulation of miR-130b, miR-100HG, and miR-1292, epigenetic modifications of E2F1 and SFTPA2, DNA methylation of MYC and RB1, and aberrant cellular functions, such as cell cycle. (B) The genetic and epigenetic progression mechanisms from normal stage to early stage LSCC can be potentially caused by inflammatory microenvironment induced by exposure to xenobiotic toxicity nicotine, dysfunctions of FGFR1, DDR1, and CHRNA5 signaling, regulation of miR-24-2 and miR-1247, lncRNA TUG1, epigenetic modification of KLF12 and ERCC1, DNA methylation of SHOX2, and aberrant cellular functions, such as DNA repair. (C) The genetic and epigenetic progression mechanisms from early stage LADC to middle stage LADC can be potentially induced by hypoxic tumor microenvironment, dysfunctions of NOTCH1 and ITGA4 (CD49d) signaling, regulation of miR-30c-2 and miR-27b, epigenetic modifications of VIM, MYH9, MDM4, and ETS1, and dysregulation of cellular functions, such as angiogenesis, ECM remodeling, and EMT. (D) The genetic and epigenetic progression mechanisms from early stage LADC to middle stage LADC can be potentially induced by hypoxic tumor microenvironment exposed to nicotine, dysfunctions of IGF-1R, ITGB1 (CD29), and TNS1 signaling, regulation of miR-106b, epigenetic modifications of HIF1 $\alpha$, IGF-1, and ETS1, DNA methylation of ITGB1, TNS1, and miR-21, and dysregulation of cellular functions, such as lymphangiogenesis, cell migration, ECM degradation. (E) The genetic and epigenetic progression mechanisms from early stage LADC to middle stage LADC can be potentially induced by the alteration of tumor microenvironment caused by hydrogen peroxide secreted by cancer cells, dysfunctions of EGFR, EPOR, and ITGB1 (CD29) signaling, regulation of miR-143HG and miR19a, epigenetic modifications of ZEB1 and RHOB, and DNA methylation of PCK1, and dysregulation of cellular functions, such as gluconeogenesis, extracellular proteolysis. (F) The genetic and epigenetic progression mechanisms from early stage LSCC to middle stage LSCC can be potentially induced by the alteration of tumor microenvironment caused by oxidative stress induced by the stimulation of nicotine derived nitrosaminoketone (NNK), dysfunctions of ERBB4, PDPN, and EMR2 signaling, regulation of miR-9-2, epigenetic modifications of GATA3, FOXL1, and CDH3, DNA methylation of PDPN, and dysregulation of cellular functions, such as proliferation, cell mobility, and cell adhesion.

of microbial immigration [43]. Though immune response is activated during infection, our results indicate TGF $\beta 1$ is secreted by cancer cells in early stage LADC (Figure 2 ) and plays a role in immunosuppression in lung cancer microenvironment [44-47], suggesting the cancer cells of LADC can prevent cytotoxic attack from lymphocytes, which are recruited to tumor microenvironment through stimulation of inflammation, by secretion of TGF $\beta 1$ in cancer microenvironment. While it has been reported that LSCC is highly correlated with exposure to tobacco smoke $[5,8,12]$, which can support our finding. Our results also found SUSD4, an inhibitor of the complement system, is activated in early stage LSCC (Figure 2). It has been suggested that cancer cells can be killed by complement. To protect cancer cells themselves from being attacked by complement, they express the soluble or membrane-bound complement inhibitors [48, 49], suggesting that cancer cells of LSCC can activate SUSD4 against complement attack in early stage.

\section{Dysfunctions of miRNA regulation, DNA methylation, epigenetic modification and microenvironment alteration contribute to the progression from normal stage to early stage LADC}

\section{The inflammatory microenvironment caused by the infection of bacteria can lead to dysregulation of EGFR and TLR4 signaling pathways to progress from normal stage to early stage LADC}

As shown in Figure 6A, our results reveal that the inflammatory environment caused by the infection of bacteria and the dysregulation of genetic and epigenetic regulations could lead to the dysregulation of EGFR and TLR4 signaling pathways, resulting in abnormal cell cycle and telomere maintenance to cause normal cells to progress to early stage LADC.
In the normal stage of lung cells near LADC cancer cells, we found that TLR4 is activated by LPS and proinflammatory factor S100A9 to trigger the inflammation signaling to modulate TFs, E2F1 and $\mathrm{Sp} 1$, to regulate cell cycle through the mediation of cell cycle-related gene $R B 1$, to cause inflammatory response and immune response through the mediation of inflammation-related genes MYC and CXCL5, and to lead to telomere maintenance by the mediation of telomere-related gene TERT. During the inflammatory response, the pro-inflammatory factor S100A10 may be secreted by the nearby alveolar macrophage. S100A10 then binds with the receptor EGFR to trigger the TF MYC modulated by SEPT3 to cause telomere maintenance by the mediation of telomere-related gene TERT. Lipopolysaccharide (LPS), a component of Gram-negative bacteria cell wall, is a strong inflammatory stimulus. TLR4, Toll-like receptor 4, is not only expressed in immune cells, but also in epithelial cells, and is an important receptor for the recognition and initiation of inflammation signals. It has been reported that LPS can also activate immune cells to trigger the production of pro-inflammatory cytokines and other mediators through TLR4 [50, 51], causing the formation of inflammatory environment. Besides, our results also showed that the protein SFTPA2, which is involved in the clearance of pathogens in response to lipopolysaccharide (LPS) [52], is activated by several epigenetic modifications in the early stage of LADC suggesting that tumor cells may affected by the pathogens. Moreover, we also observed that the ligand protein Haptoglobin (HP), which can be increased in lung cancer during infection and inflammation $[53,54]$, is activated in middle stage LADC. Based on these information, we suggested that cells may be affected by the infection of becteria during the progression from normal cells to early stage LADC. However, due to the inflammatory response caused by the infection of bacteria, the microenvironment is altered, leading to formation of inflammatory microenvironment. The microenvironment 
is altered in early stage LADC, potentially causing the dysregulation of EGFR and TLR4 signaling.

In early stage LADC, LPS can bind to the receptor TLR4 to trigger TFs MYC and Sp1 through transductance protein POLR2D, to regulate cell cycle through the mediation of cell cycle-related gene $E 2 F 1$. Besides, protein EGFL7 might be secreted via nearby cancer cells [55] to activate receptor EGFR to trigger TFs, $\mathrm{E} 2 \mathrm{~F} 1$, c-Myc, and Sp1, which are mediated by proteins SLC22A18, CDK6, RB1, PIK3R3, TERT, SFTPA2 in the corresponding signaling pathways, to regulate cell cycle through mediation of cell cycle-related genes $R B I$ and $E 2 F 1$. However, our results showed that not only the altered EGFR and TLR4 signaling pathway but also the dysfunctions of miRNA regulation, DNA methylation, and epigenetic modification cause normal cells to progress to the early stage of LADC. The epigenetic dysfunctions of miRNA regulation, DNA methylation and epigenetic modifications of LADC are described as follows.

\section{Dysregulations of miR-130b, miR-100HG, and miR- 1292, epigenetic modifications of EGFL7, SFTPA2, and E2F1, DNA methylation of MYC and RB1, and the mutation of EGFR contribute to the progression from normal stage to early stage LADC}

EGFL7, also known as VE-statin, is a secreted protein. This protein was specifically expressed by cancer cells in various human tumors and by endothelial cells in normal tissues. In embryo, EGFL7 is mainly expressed by endothelial cells; however it is downregulated in adult and has a small amount of expression in lung [47]. A vitro study showed that the expression of leukocyte adhesion molecules can be inhibited by EGFL7 through endothelial cells [56]. By preventing lymphocyte adhesion, the entry of immune cells into tumor microenvironment is limited. Our results reveal that EGFL7 is not downregulated in early stage due to the ELP3-induced acetylation, suggesting that EGFL7 plays a role in tumors immune evasion in the early stage of LADC. Besides, EGFL7 can bind to receptor EGFR to trigger oncogenic signaling to mediate abnormal cellular functions, such as cell cycle.

Moreover, the mutation of EGFR may also cause the dysregulation of EGFR signaling to induce abnormal cell cycle, which may potentially cause normal cell to progress to the early stage of LADC. Epidermal Growth Factor Receptor (EGFR), a tyrosine kinase, is involved in survival, proliferation, and tumorigenesis. It has been implicated in the growth of several human cancers, including lung cancer. Besides, the overexpression of EGFR has been found in several epithelial malignancies, including lung cancer (approximately $40-80 \%$ of NSCLC) [57]. The catalogue of somatic mutations in cancer (COSMIC) database shows that mutations in EGFR have been associated with NSCLC, including LADC (https://www. sanger.ac.uk/genetics/CGP/cosmic). Surfactant Protein
A2 (SFTPA2) is also known as SP-A. Previous studies have shown that SP-A also plays a role in the regulation of cellular responses, including inflammatory responses, by the regulation of producing pro-inflammatory cytokines in response to lipopolysaccharide to the clearance of a variety of pathogens. For example, SP-A can inhibit NO production and LPS-induced cytokine in vivo [52]. In early stage LADC, SFTPA2 is activated by USP19-induced deubiquitination and KAT5-induced acetylation. The activated SFTPA2 not only can help signaling transduction in EGFR signaling but also can allow cancer cells to clean the pathogens through expressing Surfactant Protein A2 (SFTPA2) to survive in early stage LADC.

Due to the effect of HECW2-induced ubiquitination, TF E2F1 is degraded in normal cells, resulting in the inhibition of E2F1 in normal cells. Besides, E2F1 is also inhibited by a higher expression of miR-100HG in normal stage. This allows cell cycle-related gene $R B I$ and inflammatory-related gene $M Y C$ to be transcribed to induce inflammatory response and inhibit cell cycle progression. However, the E2F1 expression is increased in early stage without the modification of ubiquitination, inducing cell cycle by the mediation of cell cycle-related gene $R B 1 . \mathrm{RB} 1$ is an important regulator of entry into cell division and can prevent excessive cell growth through the inhibition of cell cycle progression. It is known that $\mathrm{RB} 1$ is a tumor suppressor and is frequently inactivated in many cancers, including lung cancer. Our results showed that though $R B 1$ is upregulated by the regulation of degraded $\mathrm{E} 2 \mathrm{~F} 1, R B 1$ is inhibited by miR-130b in normal stage. Besides, with a lower basal level in the early stage, $R B 1$ has a significant basal level difference between normal and early stage of LADC reflecting the repressive ability on this gene might be caused by DNA methylation. According to comparing DNA methylation profiles of normal stage and early stage LADC, the significant change of methylation level in RBI ( $p$-value $<1.2 \times 10^{-23}$ ) can also support this result. Hence the abnormal cell cycle function caused by the dysregulation of RB1 can lead to the accumulation of DNA damage to potentially drive the normal cells to progress to LADC.

V-Myc Avian Myelocytomatosis Viral Oncogene Homolog (c-Myc) is the coding protein of gene $M Y C$ and involved in cell cycle progression and apoptosis. In early stage LADC, MYC is inhibited by the activated miR-1292, which may be involved in cell cycle $\left(p\right.$-value $\left.<1.2 \times 10^{-4}\right)$. Moreover, with a lower basal level in early stage, $M Y C$ has a significant basal level difference between normal and early stage of LADC reflecting that the lower expression is partly caused by the repression of DNA methylation. According to comparing DNA methylation profiles of normal stage and early stage LADC, the significant change of methylation level in MYC ( $p$-value $<1.2 \times 10^{-18}$ ) can also support this result. These indicate that MYC is inhibited in early stage LADC, leading to the upregulation of E2F1 to induce cell cycle. TERT, which encodes 
Telomerase Reverse Transcriptase, is a ribonucleoprotein enzyme and plays a role in the regulation of telomerase activity, telomere maintenance and cellular senescence. It has been reported that the aberrant expression of TERT has been found in lung cancer [58]. Our results show that TERT is inhibited by miR-130b in normal stage. However, TERT is upregulated by the TF $\mathrm{Sp} 1$ and inhibited by miR$100 \mathrm{HG}$ without the inhibition of miR-130b in early stage LADC, indicating that the dysfunction of miR-130b can cause an abnormal telomere maintenance to allow cells to become immortal to drive normal lung cells to progress to the early stage of LADC.

\section{Dysfunctions of miRNA regulation, DNA methylation, epigenetic modification and microenvironment alteration contribute to the progression from normal stage to early stage LADC}

\section{The inflammatory microenvironment caused by exposure to nicotine can lead to the dysregulation of FGFR1, DDR1, and CHRNA5 (a5 nAChR subunit) signaling pathways from normal stage to early stage of LSCC}

As shown in Figure 6B, our results reveal that the inflammatory microenvironment caused by exposure to nicotine and the dysregulation of genetic and epigenetic regulation could lead to the dysregulation of FGFR1, DDR1, and CHRNA5 signaling pathways, resulting in abnormal DNA repair, proliferation, and telomere maintenance to cause normal cells to progress to early stage LSCC.

In the normal stage of lung cells near LSCC cancer cells, receptor FGFR1 activated by FGF19, which might be secreted by nearby cancer cells, triggers the repair signaling to affect TF YBX1 through cascade proteins, RPL30 and KLF12 to regulate DNA repair by the mediation of DNA repair-related gene ERCC1. FGF19 participates in a variety of cellular processes, such as tissue repair, cell growth, morphogenesis, and, tumor growth. Recent study indicated that LSCC cell growth can be stimulated by FGF19 [59]. Moreover, the collagen COL3A1 interacts with receptor DDR1 on normal cells to trigger TF ANLN, which is modulated by proteins, KFBP5 and ERCC1, to regulate proliferation through the proliferation-related gene IGF2BP3. However, due to the inflammatory response caused by exposure to nicotine, the microenvironment is altered, leading to the formation of inflammatory microenvironment. The altered microenvironment could potentially cause the dysregulation of FGFR1, DDR1, and CHRNA5 ( $\alpha 5$ nAChR subunit) signaling pathways.

In early stage LSCC, CHRNA5, the nicotinic acetylcholine receptor subunit ( $\alpha 5 \mathrm{nAChR}$ subunit), activated by nicotine, triggers the TF YBX1 through cascade proteins, TERT and SHOX2 to regulate DNA repair and proliferation through the mediation of gene SHOX2 as shown in Figure 6B. Nicotine, a xenobiotic toxicity, is addictive substance in cigarette smoke. It has been reported that nicotine will cause oxidative stress, leading to DNA damage, and also plays a role in immunosuppressive [60-62]. Besides, nicotine can facilitate the degradation and reorganization of stromal matrix and the secretion of extracellular matrix proteins, contributing to the formation of cancer-promoting environment [60]. Collagen, the most abundant ECM protein in the matrix, can also contribute to tumor progression in the tumor matrix [63]. It has been reported that COL3A1 can promote cell proliferation, migration, and monocyte recruitment in cancer [64], suggesting that the monocyte was recruited to the microenvironment of LSCC by COL3A1 while environment was stimulated by nicotine. The receptor DDR1 is activated by collagen COL3A1 to directly trigger the TF YBX1 to regulate DNA repair by the mediation of DNA repair-related gene, $E R C C 1$ and to regulate proliferation by the mediation of proliferation-related gene, $S P 1$. In addition, receptor FGFR1 activated by the ligand FGF2 which may be secreted via nearby fibroblast is involved in cancer growth and malignant progression of NSCLC [65]. Triggered TF Sp1 through a cascade of proteins, RPL30, CIAPIN1, GSK3B, IGF2BP3, SLC6A11 in the corresponding signaling pathways regulates telomere maintenance by the mediation of telomere-related gene TERT, and both proliferation and DNA repair by the mediation of SHOX2 and $I G F 2 B P 3$. However, our results show that not only the genetic dysregulations of FGFR1, DDR1, and $\alpha 5$ $\mathrm{nAChR}$ subunit signaling pathways affected by the altered microenvironment but also epigenetic dysfunctions of miRNA regulation, DNA methylation, and epigenetic modification caused normal cells to progress to the early stage of LSCC. The epigenetic dysfunctions of miRNA regulation, DNA methylation, and epigenetic modification of LSCC are described as follows.

\section{Dysregulations of miR-24-2, miR-1247, epigenetic modifications of KLF12 and ERCC1, and DNA methylation of $\mathrm{SHOX} 2$ contribute to the progression from normal stage to early stage LSCC}

Kruppel Like Factor 12 (KLF12) has been reported that its reduced expression can promote the cell cycle G1/S transition and make the cell more susceptible to undergo apoptosis after cell detachment from matrix in tumor, suggesting that the expression of KLF12 in normal cells can allow normal cells to growth properly [66]. Based on our results in Figure 6B, we found that KLF12 is inhibited by the KAT7-induced acetylation and UBE2J1induced ubiquitination in normal stage. The inhibition of KLF12 not only can induce cell cycle G1/S transition, but also can lead to the dysregulation of FGFR1 pathway by 
interrupting the signaling transduction in normal stage, which may cause abnormal DNA repair to enhance the risk of carcinogenesis. Besides, after binding to collagen COL3A1, the acetylated receptor DDR1 enters the cytoplasm to directly interact with TF YBX1 to mediate DNA repair and proliferation. In early stage LSCC, the USP6-induced deubiquitination of YBX1 results in an activated YBX1 to regulate $E R C C 1$ and $S H O X 2$ to induce DNA repair. However, in spite of the effect of the change of DNA methylation, protein ERCC1 is degraded by the BTRC-induced ubiquitination, leading to the inhibition of DNA repair. Hence, the abnormal DNA repair may induce the accumulation of DNA damage caused by exposure to carcinogen nicotine, which may cause normal cells to progress to early stage LSCC. Y-Box Binding Protein 1 (YBX1), a cold shock domain protein, has been implicated in the regulation of various cellular functions, such as transcription and DNA repair.

Sp1, a zinc finger transcription factor of the Krüppel-like factor family, is expressed ubiquitously in various mammalian cells and is involved in the regulation of cellular processes, including cell growth, cell differentiation, immune responses, and DNA damage response [67]. Our results show that $S P 1$ is inhibited by the activated miR-24-2 and miR-1247 in normal stage. However, in the early stage of LSCC, SP1 affected by DNA methylation (Figure 2) is upregulated by the regulation of ETS1 without inhibitions from miR-24-2 and miR-1247. This indicates that proliferation can be activated by the mediation of $S P 1$, which could potentially cause normal cells to progress to early stage LSCC. Besides, it has been reported that the expression level of Sp1 is accumulated strongly higher in early stage [68], which is consistent with our result.

Moreover, our results also found that TERT is inhibited by the activated miR-1247 in normal stage, however, in early stage LSCC, TERT is upregulated by $\mathrm{Sp} 1$ in the aberrance of miR-1247 silencing, indicating that the activation of telomere maintenance through the mediation of TERT can allow normal cells to become immortal to potentially progress to early stage LSCC. In addition, in spite of the effect of the DNA methylation, $I G F 2 B P 3$ is upregulated by the positive regulation of the activated IncRNA TUG1, leading to the activation of proliferation in early stage LSCC. Moreover, lncRNA TUG1 is involved in the regulation of cancer progression. It has been reported that the downregulation of TUG1 can be observed in NSCLC [69]. However, our results showed that TUG1 plays a role in promoting LSCC proliferation. Some studies proposed that a downregulated TUG1 may potentially inhibit proliferation and cell invasion [70, 71], which can support our results. For $\mathrm{SHOX} 2$ with a lower basal level in early stage, it has a significant basal level difference between normal and early stage LSCC reflecting that the repressive ability on this gene might be caused by DNA methylation. According to comparing
DNA methylation profiles of early stage LSCC and middle stage LSCC, the significant change of methylation level in SHOX2 ( $p$-value $<6.4 \times 10^{-17}$ ) supports our result. Based on what we mentioned above, abnormal DNA repair and proliferation may lead to the accumulation of DNA damage driving normal cells to progress to early stage LSCC.

\section{Microenvironment change, dysregulation of miRNA/IncRNA, DNA methylation, and epigenetic modification contribute to genetic and epigenetic progression mechanisms from early stage to middle stage of LADC and LSCC}

As shown in Figure 5C and 5D, we identified that a hypoxic tumor microenvironment can potentially cause early stage LADC to progress to middle stage LADC, while the hypoxic tumor microenvironment exposed to xenobiotic toxicity nicotine can potentially cause early stage LSCC to progress to middle stage LSCC. In LADC, a hypoxic tumor microenvironment can potentially cause the dysregulation of NOTCH1 and ITGA4 signaling pathways. Besides, the acetylation of VIM and MYH9, acetylation of ETS1, and dysregulation of miR-30c-2 and miR-27b could also result in abnormal cellular functions, such as angiogenesis, EMT, apoptosis, ECM, and cell migration, causing early stage LADC to progress to middle stage LADC. In LSCC, a hypoxic tumor microenvironment exposed to xenobiotic toxicity nicotine can potentially cause the dysregulation of IGF-1R, ITGB1 and TNS1 signaling pathways. Moreover, the acetylation of IGF-1, ACTA2, ETS1 and HIF1 $\alpha$, the DNA methylation in ITGB1, TNS1, miR-21, and the dysregulation of miR$106 \mathrm{~b}$ and miR-21 result in abnormal cellular functions, such as proliferation, cell migration, ECM degradation, and lymphangiogenesis, causing early stage LSCC to progress to middle stage LSCC.

Interestingly, based on our results in LADC, we found that cellular functions of angiogenesis and EMT are activated during the progression from early stage to middle stage LADC. It indicates that LADC cancer cells may undergo EMT to increase migrating ability to potentially drive cancer cells to invade surrounding tissue by secreting ECM remodeling-associated protein LOX. EMT is a process inducing epithelial cells to acquire a mesenchymal phenotype. Recent study demonstrated that EMT has been observed in LADC and LSCC [72]. EMT can allow malignant epithelial cell to detach from the primary tumor to promote the ability of cancer migration. However, our results showed that EMT occurs in LADC rather than LSCC, indicating that LSCC cancer cells may promote their invasive ability by other way. Indeed, in LSCC, the ECM degradation is activated during the progression from early stage to middle stage LSCC, indicating that cancer cells of LSCC can secret ECM degradation-associated protein MMP-2 to promote 
their invasive ability. Moreover, angiogenesis is activated partly by the secretion of angiogenesis factor VEGFA during the progression from early stage to middle stage, enabling LADC cancer cells to gain more sufficient oxygen to prevent hypoxia-induced apoptosis and potentially promote the dissemination of LADC cancer cells through hematogenous metastasis. While in LSCC, lymphangiogenesis is activated by the mediation of IGF-1 during the progression from early stage to middle stage LSCC. We found that the upregulated CCR7 binding to ligand CCL21 secreted by lymph node allows cancer cells to have chemotaxis toward lymph node, indicating that cancer cells of LSCC may invade to lymph node and metastasize through lymphatic metastasis.

\section{Dysfunctions of miRNA regulation, DNA methylation, epigenetic modification and microenvironment alteration contribute to the progression from early stage to middle stage LADC}

The hypoxic tumor microenvironment can lead to the dysregulation of NOTCH1 and CD49d signaling pathways from early stage to middle stage LADC

As shown in Figure 6C, our results reveal that the hypoxic tumor microenvironment and the genetic and epigenetic dysregulation could lead to the alteration of NOTCH1 and ITGA4 signaling pathways, resulting in abnormal angiogenesis, EMT, apoptosis, ECM remodeling, and cell migration to cause early stage LADC to progress to middle stage LADC.

In early stage LADC, NOTCH1 is activated by the interaction with ligand Jagged 2 (JAG2) secreted by the nearby cancer cells and triggers TF ETS1 mediated by protein MDM4 and TF ZEB1 mediated by proteins, SLC6A20 and CDC42EP1 in the corresponding signaling pathways to regulate cell migration through the mediation of cell migration-related genes, MYH9 and miR-27b, to regulate $E C M$ remodeling through the mediation of gene $L O X$, to regulate EMT through the mediation of $L O X$ and $Z E B 1$, and to regulate angiogenesis and apoptosis through the mediation of gene $m i R-27 b$. Recent studies have reported that NOTCH1 is implicated in cancer development and progression in NSCLC and is involved in tumor initiation, growth, EMT, and metastasis [73-79]. In addition, it has been observed that NOTCH1 could suppress tumor proliferation under normoxia, while under hypoxia, NOTCH1 is involved in the promotion of tumor [80]. However, the rapid growth of cancer cells in early stage may extend beyond the oxygen diffusion limit, thus the tumor of LADC and surrounding cells in microenvironment are subjected to a low oxygen condition, causing a hypoxic tumor microenvironment. The alteration of microenvironment may cause the dysregulation of NOTCH1 and ITGA4 signaling pathways.
In middle stage LADC, HIF1A is expressed by the stimulation of hypoxia, and NOTCH1 activated by interacting with Haptoglobin, which may be secreted by the nearby cancer cells, also triggers TF HIF $1 \alpha$ to regulate angiogenesis by the mediation of angiogenesis-related gene $V E G F A$ and to regulate ECM remodeling and EMT by the mediation of gene $L O X$ (Figure 6C). Haptoglobin (HP), an acute phase plasma glycoprotein, can bind to free hemoglobin to prevent oxidative stress. HP is generated from the liver. In blood plasma, the increased amount of HP has been found in infection, inflammation, and various malignant diseases, including lung cancer $[53,54]$. Besides, it is revealed that the overexpression of HIF $1 \alpha$ is associated with the upregulated HP under the hypoxia condition in human hepatoma cells [81], suggesting that HP may be secreted by nearby cancer cells under the hypoxia. In addition, the ITGA4 activated by chemokine IL-8, which is secreted by the surrounding macrophage stimulated by hypoxia, triggers TF ETS1 modulated by proteins, Vimentin (VIM), COL22A1, MYH9, and MMP-8 in the corresponding signaling pathways, to regulate cell migration through the mediation of cell migration-related genes, $m i R-27 b$ and $J A G 2$, to regulate ECM remodeling through the mediation of gene $L O X$, to regulate EMT through the mediation of genes, $L O X$ and $Z E B 1$, and to regulate apoptosis through the mediation of genes, JAG2 and $m i R-27 b$. However, our results show that not only the dysregulation of NOTCH1 and ITGA4 signaling pathways, which are affected by the alteration of microenvironment, may be involved in the progression from early stage of LADC to progress to middle stage of LADC, but also the dysregulation of miRNA, DNA methylation, and epigenetic modification can cause early stage LADC to progress to middle stage LADC. The epigenetic dysfunctions of miRNA regulation, DNA methylation, epigenetic modification of LADC are described as follows.

\section{Dysregulations of miR-30c-2 and miR-27b, epigenetic modifications of VIM, MYH9, MDM4, and ETS1 contribute to the progression from early stage to middle stage LADC}

MDM4, also known as MDMX, is an important player in cancer progression. MDMX silencing can result in apoptosis and growth arrest in NSCLC cells [82]. Our results in Figure 6C show that the protein MDM4 is modified by the UBE2U-induced ubiquitination in early stage. MDMX can form heteroligomers with MDM2, an E3 ubiquitin ligase, to increase the MDM2 ubiquitin ligase activity to suppress the transcriptional activity of p53 by binding to $\mathrm{p} 53$ and mediate the proteasomal degradation of cell cycle-related proteins $[83,84]$. Besides, DNA damage can induce the degradation of MDMX [85]. It has been also reported that EMT phenotype is associated with an increased expression of MDMX [86]. Hence we suggest that the ubiquitination of MDM4 may lead to the growth 
arrest of LADC, allowing cancer cells to maintain in early stage, not to progress to middle stage LADC. However, the ubiquitination of MDM4 may influence on the NOTCH1 signaling pathway to cause the dysregulation of downstream signalings and functions, such as apoptosis, angiogenesis, EMT, ECM remodeling and cell migration, which may potentially drive early stage LADC to progress to middle stage LADC.

Our results also show that ETS1 is degraded by the NHLRC1-induced ubiquitination in early stage to allow cancer cells to maintain in early stage by inhibiting invasive functions, such as cell migration and ECM remodeling. However, the ATAT1-induced acetylation of ETS1 could enhance its transcription ability in middle stage to activate cell migration, ECM remodeling and EMT through the mediation of $J A G 2$ and $m i R-27 b$, the mediation of $L O X$, the mediation of $Z E B 1$ and $L O X$, respectively to potentially cause early stage LADC to progress to middle stage LADC. Zinc finger E-box binding homeobox 1 (ZEB1) is a transcription factor involved in EMT. It has been shown that the expression of ZEB1 was correlated with the mesenchymal phenotype in NSCLC and significantly correlated with the expression of VIM [87, 88]. Besides, we observed that JAG2 is inhibited by miR-30c-2 in both early and middle stage of LADC. However, the inhibited miR-30c-2 can cause the upregulation of $J A G 2$ in middle stage to induce angiogenesis and anti-apoptosis. It has been reported that in mice, JAG2 is associated with the promotion of metastasis in lung adenocarcinoma [77]. Besides, the increased expression of JAG2 is associated with hypoxia and the increased expression of JAG2 on tumor cells can promote the tube formation of endothelial cell [89], indicating the involvement of JAG2 in angiogenesis. These indicates that JAG2 is increased in middle stage to promote the angiogenesis and invasion ability of cancer cells.

Besides, our results in Figure 6C show that HIF1A is degraded by VHL-induced ubiquitination in the early stage of LADC. However, when microenvironment becomes hypoxic, HIF1A is activated by the stimulation of hypoxia, hence transcribing genes, VEGF and $L O X$ induce angiogenesis, ECM remodeling, and EMT. Hypoxia Inducible Factor 1 Alpha Subunit (HIF1 $\alpha$ ) is an important mediator of the cellular response to hypoxia and is involved in the cellular regulations of cancer cells, including angiogenesis, erythropoiesis, invasion, and metastasis [90-92]. Under normoxia, HIF $1 \alpha$ can be degraded by ubiquitin protein VHL; however, it can be stabilized under hypoxia. It has been reported that the expression of HIF1A is increased in NSCLC and the expression of HIF $1 \alpha$ is significantly associated with VEGFA [93-96]. These are consistent with our results, which can support our findings. LOX is an enzyme and can catalyze the cross-linking of ECM components, such as collagen and elastin, by oxidizing lysine residues. It has been reported that HIF $1 \alpha$ can regulate LOX to promote LOX transcription under hypoxia, and LOX can increase EMT, cell invasion, and motility, and enhance metastasis in vivo $[97,98]$. The increased expression of LOX has been observed in tissue sample from patients with LADC, and the co-expression of LOX and HIF $1 \alpha$ is associated with the decreased survival of NSCLC patients [98, 99].

Owning to the effect of modifications of VHLinduced ubiquitination and SIRT5 -induced deacetylation, VIM is decreased in early stage LADC, while VIM is increased by the modification of KAT7-induced acetylation. Vimentin (VIM), a major constituent of proteins belonging to the intermediate filament family, can provide resistance against stress and is involved in maintaining cellular integrity. VIM is a marker of the EMT $[100,101]$. During EMT, VIM can induce changes in cell motility and shape to participate in cell migration [100], suggesting that the activated VIM caused by acetylation can promote cell migration in middle stage LADC.

Myosin-9 (MYH9) is an actin-binding motor protein. In our results, MYH9 is decreased in early stage LADC and caused by UBE2U-induced ubiquitination, ESCO1-induced acetylation, and the inhibition of miR27b, while the NAT6-induced acetylation of MYH9 leads to the activation of MYH9 without the inhibition of miR-27b in middle stage LADC, though MYH9 is also affected by DNA methylation. It has been observed that the dysregulation of miR-27b has been observed in human cancers, for example, the downregulated miR-27b can be found in lung adenocarcinoma [102, 103]. MiR-27b could inhibit the invasion, migration, and proliferation in cancer cells [104]. Besides, it has been shown that miR-27b can increase the vascularization and growth of subcutaneous tumors significantly, for example, miR-27b can promote the angiogenesis and cancer growth in lung cancer [105]. Recently studies have shown that MYH9 participates in cancer cell migration, invasion, and metastasis, and MYH9 expression was significantly correlated with the adenocarcinoma histology [106-108], suggesting that the activation of MYH9 ( $p$-value $<4.4 \times 10^{-2}$ ) caused by acetylation can promote cell migration in middle stage LADC and may potentially increase LADC invasion and metastasis.

\section{Dysfunctions of miRNA regulation, DNA methylation, epigenetic modification and microenvironment alteration contribute to the progression from early stage to middle stage LSCC}

\section{The hypoxic tumor microenvironment exposed to nicotine can lead to the dysregulation of IGF-1R, TNS1, and ITGB1 (CD29) signaling pathways from early stage to middle stage LSCC}

As shown in Figure 6D, our results reveal that the hypoxic tumor microenvironment exposed to xenobiotic 
toxicity nicotine and the dysfunction of genetic and epigenetic regulation could lead to dysregulation of IGF$1 \mathrm{R}$ and ITGB1 signaling pathway and the dysregulation of focal adhesion molecule TNS1 signaling, resulting in abnormal proliferation, ECM degradation, cell migration, and lymphangiogenesis to cause early stage LSCC to progress to middle stage LSCC.

In early stage, the focal adhesion molecule tensin 1 (TNS1) interacts with the extracellular matrix protein COL1A1, which is a collagen to trigger the TFs JUN, GATA2, and ETS1 via cascade proteins ACTA2, CDKN2A, and PCDP1 in the corresponding signaling pathway and to regulate both the proliferation through the mediation of proliferation-related genes $m i R-21$, $m i R-106 b$, and IGF1 and the lymphangiogenesis through the mediation of gene IGF1. In previous microarray analyses, the upregulated COL1A1 was found in various malignant tissues, including lung cancer [109]. Besides, the overexpression of COL1A1 is correlated with hypoxic conditions and the strong correlation has been observed between its expression and hypoxia markers [110]. CDKN2A expressed in the cytoplasm and nucleus can form a complex with other proteins such as actin and tubulin depending on post-translational modifications [111, 112], which can support our results; the deacetylated CDKN2A can interact with the acetylated ACTA2 to promote cell migration. However, similar to the NSCLC subtype LADC, the rapid cell proliferation could lead to cancer cells in early stage to extend beyond the oxygen diffusion limit. Therefore, the LSCC cancer cells and cells surrounding in microenvironment are subject to a low oxygen condition, causing a hypoxic tumor microenvironment. Besides, a hypoxic tumor microenvironment is also exposed to xenobiotic toxicity nicotine. The alteration of microenvironment could cause the dysregulation of TNS1, IGF-1R and CD29 signaling pathways.

In middle stage LSCC, HIF1A is activated. It has been shown that hypoxia-inducible factor $1 \alpha(\mathrm{HIF} 1 \alpha)$ can be activated by the stimulation of hypoxia and also by the stimulation of nicotine in lung cancer [113], suggesting HIF $1 \alpha$ is expressed by the stimulation of both hypoxia and xenobiotic toxicity nicotine in middle stage LSCC. The activated HIF $1 \alpha$ then binds with TFs, JUN and ETS1, to regulate proliferation and lymphangiogenesis by the mediation of proliferation-related gene miR21 and IGF1, which is also involved in lymphangiogenesis. After IGF1 binding to receptor IGF-1R secreting caused by the stimulation of hypoxia, it triggers TFs JUN, ETS1, JunB, and GATA2 through cascade proteins SUSD4, CDKN2A, and PCDP1 in the corresponding signaling pathways to regulate ECM degradation through the mediation of $M M P 2$, proliferation through mediation of $m i R-21, m i R-$ 106 , and $I G F 1$, lymphangiogenesis through the mediation of $I G F 1$, and cell migration through the mediation of TNS1 (Figure 6D).
In addition, when CCL13 binding to ITGB1, CD29 then directly triggers the TF JUN. TF c-Jun then interacts with ETS1 to regulate cell migration by the mediation of $C C R 7$, and ITGB1. It has been reported that the induction of senescence in cells can cause an increased expression of chemokines, such as CCL13 [114]. Besides, bronchial epithelial cell can induce apoptosis and senescence through ROS-mediated autophagy-impairment by exposure to nicotine. Hence we suggest that CCL13 may be secreted by nearby bronchial epithelial cells, which are induced by senescence under nicotine exposure. ETS1, Ets1 proto-oncoprotein, is a member of the Ets family of transcription factors and is involved in the regulation of several cancer-associated functions, such as ECM remodeling, cell migration, and proliferation $[115,116]$. It has been reported that ETS1 can enhance transcription synergistically with AP-1 (c-Jun) by interacting with AP-1 (c-Jun) to form complex [117], which can support our results; TF JUN can interact with ETS1 to regulate cell migration by the mediation of CCR7 and ITGB1. However, our results show that not only the dysregulation of TNS1, IGF-1R and ITGB1 signaling pathways, which are affected by the altered microenvironment, may be involved in the progression from early stage to middle stage LSCC, but also the dysfunctions of miRNA regulation, DNA methylation, and epigenetic modification can cause early stage LSCC to progress to middle stage LSCC. The epigenetic dysfunctions of miRNA regulation, DNA methylation, epigenetic modification of LSCC are described as follows.

\section{Dysregulation of miR106b, epigenetic modifications of HIF1a, IGF-1, and ETS1, and DNA methylation of ITGB1, TNS1, and miR21 contribute to the progression from early stage to middle stage LSCC}

In early stage LSCC, the UBE2Q1-induced ubiquitination of ETS1 leads to the downregulation of $I G F 1$, resulting in the inhibition of lymphangiogenesis as shown in Figure 6D. However, the NAT9-induced acetylation of ETS1 in middle stage LSCC leads to the upregulation of cell migration-related genes, CCR7 and ITGB1, and lymphangiogenesis-related gene $I G F 1$, resulting in the activation of cell migration and lymphangiogenesis. The activation of cell migration and lymphangiogenesis may promote the invasion ability of cancer cells and potentially allow cancer cells to undergo lymphatic metastasis in the middle stage of LSCC.

IGF-1R, a transmembrane heterotetrameric protein, can promote cancer cell proliferation, survival and transformation toward malignancy. IGF-1, an insulinlike growth factor, can be delivered not only from distant sources, but also through paracrine/autocrine signalings in aggressive tumors [118]. Through receiving the acetylated IGF-1 signaling, IGF-1R is activated to regulate cancerassociated functions, such as the proliferation to promote 
cancer cell proliferation. It has been shown that IGF1R can be stimulated by its ligand insulin-like growth factor 1 (IGF-1) [119]. The aberrant IGF signaling has been observed in several cancers, including lung cancer [120]. Besides, the increased IGF-1R activity is implicated in cancer cell function such as cell migration, proliferation, and invasion $[118,120]$. In our study, we found that HIF $1 \alpha$ is inhibited by UBE2Z-induced ubiquitination and HDAC5-induced deacetylation. However, in middle stage LSCC, HIF $1 \mathrm{~A}$ is activated by the stimulation of hypoxia and nicotine [113]. In addition, HIF $1 \alpha$ is also affected by NAT8-induced acetylation. The activated HIF $1 \alpha$ then interacts with both JUN and ETS1, which are also affected by NAT9-induced acetylation, to upregulate $I G F 1$. It has been shown that the accumulation of HIF $1 \alpha$ under hypoxia can lead to an increased production of IGF-1 [121], which can support our findings. In addition, it has been reported that IGF-1 is associated with lymphangiogenesis [122], suggesting that lymphangiogenesis is occurred in middle stage LSCC as shown in Figure 6D.

CCR7 is a chemokine receptor. The expression of CCR7 has been observed and involved in the invasion and metastasis of NSCLC [123]. It has been reported that lymph nodes can produce CCL21 and the interaction between CCL21 and CCR7 can regulate the directional migration to promote the lymph node metastasis of breast cancer [124], suggesting that CCR7 is involved in cell migration. In our result, though $C C R 7$ is inhibited by miR-21 in early stage LSCC and CCL21 expression is decreased in middle stage LSCC, cancer cells through the upregulation of $C C R 7$ in middle stage LSCC to increase the binding probability to interact with chemoattractant CCL21 can promote themselves to migrate to lymph nodes (Figure 3 ). Taken together, the production of IGF-1 and the activation of expression of CCR7 result in lymphangiogenesis and may promote cell migration toward lymph node in middle stage LSCC, suggesting that cancer cells may metastasize through lymph node in LSCC.

ACTA2 belongs to the actin family of proteins and is involved in the regulation of cell movement and maintenance of cell shape. The down regulation of ACTA2 could remarkably impaire in vitro cell invasion and migration in LADC [125]. Our results indicate that ACTA2 is activated by the CREBBP-induced acetylation, which can potentially promote the invasive ability of cancer cells by the activation of cell migration in early stage LSCC. In addition, Tensin1 (TNS1), a focal-adhesion molecule, is involved in the negative regulation of cell migration, and the reduced expression of TNS1 has been observed in human breast carcinoma, head and neck squamous cell carcinoma [126]. Our results show that TNS1 is inhibited by a lower expression of miR-21 in early stage LSCC. However, with a lower basal level in middle stage, TNS1 has a significant basal level difference between early and middle stage LSCC reflecting that the repressive ability on this gene might be caused by DNA methylation, resulting in the inhibition of TNS1 in middle stage LSCC, and suggesting that the downregulated TNS1 can reduce the inhibition of miR-21 to induce cell migration. According to comparing DNA methylation profiles of early stage and middle stage LSCC, the significant change of methylation level in TNS1 ( $p$-value $\left.<6.8 \times 10^{-3}\right)$ can also support this result.

MiR-21 plays a role in tumorigenesis, tumor cell proliferation, and invasion. With a higher basal level in middle stage, $m i R-21$ has significant basal level difference between early and middle stage LSCC. In other words, $m i R-21$ may be activated by DNA methylation resulting in the higher expression in middle stage LSCC, which indicates the activation of miR-21 can promote cancer cells invasion and proliferation in middle stage LSCC. According to comparing DNA methylation profiles of early stage and middle stage LSCC, the significant change of methylation level in miR-21 ( $p$-value $<2.6 \times$ $10^{-2}$ ) can support this result. Matrix Metallopeptidase 2 (MMP-2) plays a key role in the promotion of cancer invasion and metastasis through the degradation of ECM to allow cancer cells to move around. Our results show that MMP2 is inhibited by miR-106b in early stage LSCC. While in middle stage LSCC, MMP2 is upregulated by the regulation of TF JunB in the aberrance of miR-106b and is also affected by DNA methylation, suggesting that the dysfunction of miR-106b can cause the abnormal ECM degradation to potentially promote the invasion and metastasis of cancer cells.

ITGB1, Integrin Subunit Beta 1, encodes protein CD29 and plays an important role in cancer cell motility, survival, and attachment. It has been shown that the inhibition of ITGB1 in vitro and in vivo can decrease lung cancer invasion and metastasis $[127,128]$. In our results, with a higher basal level in middle stage, ITGB1 has a significant basal level difference between early and middle stage LSCC reflecting that the active ability on this gene might be caused by DNA methylation, indicating that the upregulation of ITGBI caused by DNA methylation can induce cell migration in middle stage LSCC. According to comparing DNA methylation profiles of early stage and middle stage LSCC, the significant change of methylation level in ITGBI ( $p$-value $\left.<5.9 \times 10^{-3}\right)$ can also support this result.

\section{Microenvironment change, dysregulation of miRNA/IncRNA regulation, DNA methylation, and epigenetic modification contribute to genetic and epigenetic progression mechanisms from middle stage to progress to advanced stage of LADC and LSCC}

As shown in Figure 5E and 5F, we identified that the altered tumor microenvironment caused by hydrogen peroxide secreted by cancer cells or cancer associated 
fibroblasts (CAFs) can potentially cause middle stage LADC to progress to advanced stage LADC, while the altered tumor microenvironment caused by oxidative stress induced by the stimulation of nicotine derived nitrosaminoketone (NNK) can potentially cause middle stage LSCC to progress to advanced stage LSCC. In LADC, the altered tumor microenvironment caused by hydrogen peroxide can potentially cause the dysregulation of EGFR, EpoR, and ITGB1 (CD29) signaling pathways (Figure $6 \mathrm{E}$ ). Besides, the acetylation of ZEB1, deacetylation of RHOB, dysregulation of miR$143 \mathrm{HG}$, and miR-19a, and DNA methylation of PCK1 could also result in abnormal cellular functions, such as proliferation, gluconeogenesis, cell migration, and extracellular proteolysis, causing middle stage LADC to progress to advanced stage LADC. In LSCC, the altered tumor microenvironment caused by oxidative stress induced by the stimulation of NNK can potentially cause the dysregulation of ERBB4, PDPN, and EMR2 signaling pathways (Figure 6E). Moreover, the ubiquitination and deacetylation of GATA3, deacetylation of FOXL1, and deubiquitination of $\mathrm{CDH} 3$, dysregulation DNA methylation in $P D P N$, and dysregulation of miR-92 could also result in abnormal cellular functions, such as proliferation, cell mobility, cell adhesion, and lymphangiogenesis, causing middle stage LSCC to progress to advanced stage LSCC.

Interestingly, based on our results in LADC, we found that gluconeogenesis is activated during the progression from middle stage to advanced stage LADC. It has been reported that the tumor cells have a lower concentration of glucose about 3 times compared to the corresponding normal tissues [129]. For maintaining their cellular functions such as cell proliferation and migration, cancer cells activate gluconeogenesis to gain the energy. Besides, similar to the progression from early stage to middle stage, cell migration through EMT has also been observed by the activated $\mathrm{CDH} 2$, a mesenchymal cadherin involved in the EMT. Through secreting PLAU by cancer cells, the surrounding matrix could be reorganized via proteolysis, hence allowing LADC cancer cells to invade and metastasize to other locations. In LSCC, we found LSCC cancer cells may undergo a collective cell migration (collective cell migration associated proteins $\mathrm{CDH} 3$ and PDPN in Figure 6E) to invade or metastasize to the surrounding or other tissue. Since the migrating squamous cell carcinoma can usually be observed to form large complexes of cells during the progression of lung cancer [130], it is not surprising that the collective cell migration has been implicated in LSCC during the progression from middle stage to advanced stage LSCC in our results, From our results, we showed that LADC and LSCC can undergo different ways to move in different cancer microenvironment. The LADC cancer cells can mediate EMT-associated genes to undergo EMT, indicating that the LADC cancer cells may invade or metastasize to other sites through single cell migration, while the LSCC cancer cells may invade or metastasize to other sites through clustered cells migration (collective cell migration) through the mediation of collective migration associated proteins.

\section{Dysfunctions of miRNA regulation, DNA methylation, epigenetic modification and microenvironment alteration contribute to the progression from early stage to middle stage LADC}

The hydrogen peroxide secreted by cancer cells leads to the alteration of tumor microenvironment, causing dysregulation of EGFR, EPOR and ITGB1 (CD29) signaling pathways to progress from middle stage to advanced stage of LADC

As shown in Figure 6E, our results reveal that the altered tumor microenvironment caused by hydrogen peroxide secreted by cancer cells and the dysregulation of genetic and epigenetic regulation could lead to the alteration of EGFR, EPOR, and CD29 signaling pathways, resulting abnormal proliferation, gluconeogenesis, cell migration, and extracellular proteolysis to cause early stage LADC to progress to middle stage LADC.

In middle stage LADC, receptor EGFR can be activated by interacting with ligands EGF, which may be secreted by nearby macrophage stimulated by hypoxia, and BGN, which may be secreted by tumor endothelial cells. The activated receptor EGFR then triggers the TF Androgen Receptor (AR) through protein PCK1 and TF MYC through cascade proteins RHPN2, MTOR, and EIF4B in the corresponding signaling pathways, to regulate proliferation through the mediation of proliferation-related gene $E I F 4 B$. However, the secretion of the hydrogen peroxide by nearby cancer cells and cancer-associated fibroblasts (CAFs) could cause the DNA damage, inflammation to the altered tumor microenvironment, and cancer metabolism [131-135]. It has been reported that hydrogen peroxide is a carcinogen and may function as "fertilizer" to result in the promotion of tumor growth, progression and metastasis [136]. After being affected by hydrogen peroxide, the altered microenvironment could cause the dysregulation of EGFR, EpoR, and ITGB1 (CD29) signaling pathways.

In advanced stage LADC in Figure 6E, receptor EPOR binds to its ligand Erythropoietin (EPO) to trigger TF STAT3 through cascade proteins RHOB and XRCC4 in the corresponding signaling pathways, to regulate proliferation through the mediation of $R H O B$. Erythropoietin (EPO) is a $30.4-\mathrm{kD}$ glycoprotein. It has been reported that hydrogen peroxide $\left(\mathrm{H}_{2} \mathrm{O}_{2}\right)$ participates in the stimulation of EPO production and EPO expression regulation in the hepatocellular cells [137]. EpoR is a member of the cytokine receptor family. The human and experimental 
studies have revealed that EPO and EpoR can co-express in a diversity of human malignancies and the EPO/EpoR signaling is involved in cancer cell proliferation, inhibition of apoptosis, and invasiveness [138].

In addition, ITGB1 is activated by the interaction with ligand BGN secreted from nearby tumor endothelial to directly trigger the TF MYC and to indirectly trigger TF ZEB1 through cascade proteins, IQGAP1, CNIH4, and RHPN2 in the corresponding signaling pathways, to regulate cell migration through the mediation of $m i R$ $19 a$ and $\mathrm{CDH} 2$, to regulate gluconeogenesis through the mediation of $P C K 1$, to regulate extracellular proteolysis through the mediation of PLAU, and to regulate proliferation through the mediation of $E I F 4 B$ and $R H O B$. Biglycan (BGN) is a small leucinerich repeat proteoglycan family of proteoglycans. In Figure $6 \mathrm{E}$, BGN can be secreted from tumor endothelial cells (TECs) and is involved in cell migration, matrix assembly, and adhesion to promote lung tumors metastasis by enhancing tumor cell intravasation [139-142]. MiR-19a is involved in the regulation of cancer functions, for example, miR-19a can enhance cell invasion and migration, cell growth, and viability in NSCLC [143]. However, our results show that not only the dysregulation of EGFR, EPOR, and ITGB1 signaling pathways, which are affected by the alteration of microenvironment, but also the dysfunctions of miRNA regulation, DNA methylation, and epigenetic modification can cause middle stage LADC to progress to advanced stage LADC. The epigenetic dysfunctions of miRNA regulation, DNA methylation, and epigenetic modification of LADC are described as follows.

\section{Dysregulations of miR-143HG and miR-19a, epigenetic modifications of ZEB1 and RHOB, and DNA methylation of PCK1 contribute to the progression from middle stage to advanced stage LADC}

The plasminogen activator (PLAU), uPA, can mediate proteolysis and lead to cancer cell invasion and metastasis. Our results show that PLAU is inhibited by miR-134HG in middle stage LADC. However, in advanced stage LADC, $P L A U$ is upregulated in the aberrant of miR-143HG silencing, suggesting that the dysfunction of miR-143HG can lead to the activation of extracellular proteolysis through the mediation of PLAU. Therefore, we revealed that the matrix surrounding cancer cells can be degraded by proteolysis regulated by PLAU. This allows cancer cells to invade surroundings easily to promote cancer cells metastasis (Figure 6E). In advanced stage LADC, the ESCO1-induced acetylation of ZEB1 leads to the activation of proliferation, gluconeogenesis, cell migration, and extracellular proteolysis through the upregulation of proliferation- related gene $E I F 4 B$, extracellular proteolysis-related gene $P L A U$, gluconeogenesis-related gene $P C K 1$, and migration-related gene $\mathrm{CDH} 2$, indicating that these activated cellular functions caused by the acetylation of ZEB1 can promote LADC cancer cells invasion and metastasis.

$\mathrm{CDH} 2$, a member of the cadherin superfamily, which is known as N-cadherin, is a mesenchymal cadherin involved in the EMT and metastasis of cancer cells [144]. It has been shown that the expression of $\mathrm{CDH} 2$ may potentially cause mesenchymal phenotype by EMT and the promotion of the survival of lung cancer cells with drugresistant $[145,146]$, suggesting that $\mathrm{CDH} 2$ is a potential therapy target for anti-cancer cells. PCK1, an isoform of PEPCK, is involved in the control of gluconeogenesis [147]. Our results show that $P C K 1$, with a higher basal level in advanced stage LADC, has a significant basal level difference between middle stage and advanced stage LADC reflecting that the active ability on this gene might be caused by DNA methylation. In other words, it suggests that the upregulated $P C K 1$ caused by DNA methylation can increase gluconeogenesis in middle stage LADC. Gluconeogenesis plays a key role in development of cancer cells. Due to high consumption, cancer cells face the limitation of nutrients, such as glucose. Comparing to the corresponding normal tissues, the concentration of glucose is lower about 3 times in tumor cells [129]. However, cancer cells need energy to maintain their function such as proliferation and cell migration. Hence cancer cells need gluconeogenesis to adapt and survive in the change of microenvironment.

Besides, our results also show that RHOB is repressed in middle stage by deacetylation. While in advanced stage LADC, RHOB is also inhibited by a higher expression of miR-19a. The small GTP binding protein RHOB belongs Rho protein family and is involved in the regulation of diverse cellular processes including cell cycle progression, cytoskeletal organization, cytokinesis, and gene transcription $[148,149]$. The downregulated $R H O B$ has been observed in lung cancer, leading to the loss of its expression [150-152]. In lung cancer cell lines, the ectopic expression of RHOB can suppress anchorage-independent growth and cell proliferation [150]. It has been suggested that RHOB expression is controlled by epigenetic regulation. Wang et al. demonstrated that the repression of RHOB expression is caused by histone deacetylase 1 (HDAC1) in lung cancer cell lines [152]. Besides, recent study showed that the significant RHOB re-expression is induced by histone deacetylase (HDAC) inhibitors in lung cancer cell lines, indicating the expression of RHOB is mainly regulated by deacetylation and can support out results. Hence we suggest that RHOB is inhibited in both middle stage and advanced stage LADC to induce abnormal proliferation by the epigenetic regulation of deacetylation and dysfunction of miR-19a, respectively. 


\section{Dysfunctions of miRNA regulation, DNA methylation, epigenetic modification and microenvironment alteration contribute to the progression from middle stage to advanced stage LSCC}

\section{The oxidative stress induced by the stimulation of nicotine derived nitrosaminoketone (NNK) can potentially lead to the alteration of microenvironment, causing dysregulation of ERBB4, PDPN, and EMR2 signaling pathways from middle stage to advanced stage LSCC}

As shown in Figure 6F, our results reveal that the oxidative stress induced by the stimulation of nicotine derived nitrosaminoketone (NNK) and the dysregulation of genetic and epigenetic regulation could lead to the dysregulation of ERBB4, PDPN, and EMR2 signaling pathways, resulting in abnormal proliferation, gluconeogenesis, cell migration, and extracellular proteolysis to cause middle stage LSCC to progress to advanced stage LSCC.

In middle stage LSCC in Figure 6F, EMR2 is activated by interaction with ligand DDX5 under the hypoxia. The activated EMR2 then triggers TF FOXL1 through cascade proteins CHD3 and AGR3 in the signaling pathway, to regulate proliferation and cell mobility through the mediation of NF1. The DEADbox-protein DDX5, an ATP-dependent RNA helicase, has been involved in the promotion of proliferation and tumorigenesis in NSCLC [153]. CHD3, a catalytic component of histone deacetylase complex, is involved in tumorigenesis and metastasis [154]. Besides, under the microenvironment stresses and DNA damage, anterior gradient protein 3 (AGR3) can promote the survival of tumor cells and may participate in the coordination of cell proliferation, survival, and metastasis $[155,156]$. After binding to TGFB1, receptor ERBB4 is activated to trigger TFs YBX1 and GATA3 through cascade proteins TGFB1, CDC42, LIN7C, FN1, and NF1 in the corresponding signaling pathways. These TFs would regulate cell mobility through the mediation of $P D P N$, cell adhesion through mediation of $\mathrm{CDH} 3$, and proliferation through the mediation of $A G R 3$. However, tumor microenvironment is altered due to oxidative stress induced by stimulation of NNK, a lung carcinogen. The altered microenvironment, causing the alteration of ERBB4, PDPN, and EMR2 signaling pathway (Figure 6F).

In advanced stage LSCC, the stress-responsive transcription factor FOXO3 (also known as FOXO3A) is activated. It has been reported that the Forkhead box type-O (FOXO) family of transcription factors can respond to these stresses, including DNA damage and oxidative stress [157-160]. Evidence suggests that FOXO3A is involved in the regulation of cellular response to these stresses, such as DNA repair, apoptosis, and growth arrest [161-163]. It has been demonstrated that FOXO3A can be activated in response to nicotinederived nitrosaminoketone (NNK), a carcinogen, and plays a role in anti-carcinogenic response by the suppression of NNKinduced DNA damage in LADC [161], suggesting that FOXO3A is activated in response to NNK in advanced stage LSCC. ERBB4 is activated by interacting with COL18A1, which also interacts with FN1, to trigger TF GATA3 by the modulation of protein TGFB1. Besides, the activated receptor ERBB4 can also affect glycoprotein PDPN to trigger TFs, GATA3 and FOXO3 through cascade proteins $\mathrm{CHIC} 2$, Moesin (MSN), TUBB3, RHOXF2, SMG1 in the signaling pathways, to regulate proliferation through the mediation of FOXO3, SMG1, and $N F 1$, to regulate cell mobility through the mediation of $N F 1$, to regulate cell adhesion through the mediation of $C D H 3$, and to regulate lymphangiogenesis through the mediation of $V E G F C$ (Figure 6F). COL18A1 encodes Type XVIII collagen, also known as Endostatin, a potent antiangiogenic protein that is able to inhibit angiogenesis and is associated with malignant pleural effusions in patients of lung cancer $[164,165]$. Besides, COL18A1 is a regulator of the oxidative stress response and has been suggested that the potential links between ECM and oxidative stress response in cancer. However, our results show that not only the dysregulation of ERBB4, EMR2, and PDPN signaling pathways which are affected by the alteration of microenvironment but also the dysfunctions of miRNA regulation, DNA methylation, and epigenetic modification may be involved in the progression from middle stage to advanced stage LSCC. The epigenetic dysfunctions of miRNA regulation, DNA methylation, and epigenetic modification of LSCC are described as follows.

Dysregulation of miR-9-2, epigenetic modifications of GATA3, FOXL1, and CDH3, and DNA methylation of PDPN contribute to the progression from middle stage to advanced stage LSCC

In middle stage LSCC in Figure 6F, TF GATA3 is modified by the SIAH1-induced ubiquitination and HDAC5-induced deacetylation, leading to the degraded GATA3 to inhibit cell adhesion and proliferation through the mediation of $C D H 3$ and $A G R 3$. However, in advanced stage LSCC, GATA3 is not affected by ubiquitination and deacetylation, hence causing the activation of GATA3 in advanced stage LSCC to activate lymphangiogenesis and proliferation through the mediation of VEGFC, SMG1, and $\mathrm{FOXO3}$, indicating that these activated functions may increase the risk of lymphatic metastasis. Serine/ threonine protein kinase 1 (SMG 1) belongs to the phosphatidylinositol 3 kinase related kinase family and the knockdown expression of SMG1 can result in the inhibition of tumor cell proliferation in cancer [166].

FOXL1 belongs to the forkhead/winged helix-box (FOX) family and plays an important role in the regulation 
of cell proliferation. Our results show that the HDAC9induced deacetylation of FOXL1, which is also indirectly modified by the CHD3-induced deacetylation via AGR3, results in the inhibition of FOXL1. Hence, cell mobility and proliferation are activated by the downregulation of $N F 1$ via the inhibited FOXL1 in middle stage LSCC. It is noted that this promote middle stage cancer cells to advanced stage LSCC cancer cells potentially. Neurofibromatosis type 1 (NF1) plays negative regulatory role in the downstream of EGFR signaling and Ras cellular proliferation pathways [167-170]. Besides regulating cell proliferation, NF1 is also involved in the regulation of actin cytoskeletal reorganization to affect cell adhesion and motility. Moreover, NF1 siRNA can cause morphological change, which is the excessive formation of actin stress fiber [171].

Moreover, our results also show that the podoplanin (PDPN) is inhibited in middle stage caused by the inhibition of activated miR-199b and HERC4induced ubiquitination. While in advanced stage LSCC, PDPN is activated by the modification of SIRT1-induced deacetylation and DNA methylation. Our results reveal that PDPN, with a higher basal level in advanced stage LSCC, has a significant basal level difference between middle stage and advanced stage LSCC reflecting that the activity ability of this gene might be caused by DNA methylation. $P D P N$ encodes a type-I integral membrane glycoprotein, and its expression is upregulated in squamous cell carcinoma of lung [172]. Previous study indicates that PDPN participates in the regulation of cell motility and cytoskeletal organization by mediating ERM proteins [173]. Our results in Figure 6F indicated that membrane glycoprotein PDPN can trigger the ERM protein MSN through $\mathrm{CHIC} 2$ to facilitate cell motility and cytoskeletal organization, which can be supported by previous study. In addition, the expression of PDPN by tumor cells can induce tumor lymphangiogenesis and increase lymph nodes metastasis [174]. Moreover, it has been reported that PDPN can mediate collective cell migration (CCM) to promote tumor invasion without EMT [173].

In addition, $\mathrm{CDH} 3$ (P-cadherin) is inhibited by miR330 silencing and the regulation of GATA 3 in middle stage LSCC. While in advanced stage LSCC, CDH3 is activated by the modification of OTUD1-induced deubiquitination, though it is also affected by DNA methylation. CDH3, which belongs to the cadherin superfamily, is a classical cell-to-cell adhesion molecule. It has been observed that the overexpression of $\mathrm{CDH} 3$ (P-cadherin) is associated with the aggressiveness, invasion, and metastasis of cancer cells [175-179]. Besides, recent studies indicate that $\mathrm{P}$-cadherin can promote collective cell migration (CCM) and the depletion of P-cadherin in epithelial cells could significantly induce the impair of CCM in 2D and 3D culture systems, in in vitro [180-182]. Our results demonstrate that both $\mathrm{CDH} 3$ and PDPN are activated in advanced stage LSCC, suggesting that LSCC cancer cells may induce collective migration (CCM) to invasion and metastasis.

Moreover, FOXO3 can respond to nicotine-derived nitrosaminoketone (NNK) and is activated to inhibit proliferation through the mediation of $N F 1$. However, FOXO3 affected by DNA methylation is inhibited by the activated miR-9-2 in advanced stage LSCC. The inhibited FOXO3 then leads to the downregulation of NF1, causing the activation of proliferation and cell mobility. Hence, it promotes growth, invasion and metastasis in LSCC cancer cells. EMR2, a member of class B sevenspan transmembrane (TM7) subfamily, is modified by USP20-induced deubiquitination. It has been reported that the expression of EMR2 is associated with invasive carcinomas and may play a key role in the contribution of invasive phenotype $[183,184]$. Hence the modification of deubiquitination may lead to the dysregulation of EMR2 signaling, causing the abnormal proliferation and cell mobility to induce the progression from middle stage to advanced stage LSCC.

As discussed in the above, we summarized the differential progression genetic and epigenetic mechanisms in LADC and SCC in Figure 5, indicating that the progression from normal stage to early stage LADC can be potentially caused by inflammatory microenvironment induced by bacteria infection (LPS), the dysfunctions of EGFR and TLR4 signaling, the regulation of $\mathrm{miR}-130 \mathrm{~b}$, miR-100HG, and miR-1292, the epigenetic modifications of E2F1 and SFTPA2, the DNA methylation of $M Y C$ and $R B 1$, and the aberrant cellular functions, such as cell cycle. While the progression from normal stage to early stage of LSCC can be potentially caused by the inflammatory microenvironment induced by the exposure to xenobiotic toxicity nicotine, the dysfunctions of FGFR1, DDR1, and CHRNA5 signaling, the regulation of miR-24-2 miR-1247, and lncRNA TUG1, the epigenetic modifications of KLF12 and ERCC1, the DNA methylation of $\mathrm{SHOX2}$, and the aberrant cellular functions, such as DNA repair. The progression from early stage to middle stage LADC can be potentially induced by the hypoxic tumor microenvironment, the dysfunctions of NOTCH1 and ITGA4 (CD49d) signaling, the regulation of $\mathrm{miR}-30 \mathrm{c}-2$ and $\mathrm{miR}-27 \mathrm{~b}$, the epigenetic modifications of VIM, MYH9, MDM4, and ETS1, and the dysregulation of cellular functions, such as angiogenesis, ECM remodeling, and EMT. While the progression from early stage to middle stage LADC can be potentially induced by the hypoxic tumor microenvironment exposed to nicotine, the dysfunctions of IGF-1R, ITGB1 (CD29), and TNS1 signaling, the regulation of miR-106b, the epigenetic modifications of HIF $1 \alpha$, IGF-1, and ETS1, the DNA methylations of ITGB1, TNS1, and miR-21, and the dysregulation of cellular functions, such as lymphangiogenesis, cell migration, and ECM degradation. Besides, the progression from early stage to middle stage LADC can be potentially induced by the alteration of 
tumor microenvironment caused by hydrogen peroxide secreted by cancer cells, the dysfunctions of EGFR, EPOR, and ITGB1 (CD29) signaling, the regulation of miR-143HG and miR-19a, the epigenetic modifications of ZEB1 and RHOB, the DNA methylation of PCK1, and the dysregulation of cellular functions, such as gluconeogenesis and extracellular proteolysis. While the progression from early stage to middle stage LSCC can be potentially induced by the alteration of tumor microenvironment caused by oxidative stress induced by the stimulation of nicotine derived nitrosaminoketone (NNK), the dysfunctions of ERBB4, PDPN, and EMR2 signaling, the regulation of miR-9-2, the epigenetic modifications of GATA3, FOXL1, and CDH3, the DNA methylation of $P D P N$, and the dysregulation of cellular functions, such as proliferation, cell mobility, and cell adhesion.

\section{Identification of network biomarkers for the discovery of genetic and epigenetic multiple drugs for therapeutic treatment of early stage, middle stage, and advanced stage LADC and LSCC}

According to the genetic and epigenetic progression mechanisms based on LADC and LSCC core signaling pathways between connective stages (normal stage, early stage and middle stage), we could identify network biomarkers based on the proteins with significant differential expression change between later stage and normal stage. We then found some significant network biomarkers as follows: the proteins, SLC22A18, TERT, SEPT3, RET, POLR2D, E2F1, which have significant differential expression change with higher expression in early stage compared to normal stage, and proteins, PDGFB, EGFL7, S100A10, PDGFRA, TLR4, TLR4, CCL2, PER1, RB1, TGFB1, PIK3R3, SFTPA2, MYC, which have significant expression change with lower expression in early stage compared to normal stage (Figure 2), are selected as biomarkers in core signaling pathways between normal stage and early stage LADC; the proteins, HP, LTA, APH1A, COL22A1, MMP8, IGF2BP1, HIF1A, PRDM14, which have significant expression change with higher expression in middle stage compared to normal stage, and proteins, NOTCH1, HGF, ITGA4, VIM, SLC6A20, MYH9, ETS1, ZEB1, which have significant expression change with lower expression in middle stage compared to normal stage (Figure 3), are selected as biomarkers in core signaling pathways between early stage and middle stage LADC; the proteins, EGF, CDH2, LOXL2, RHPN2, XRCC4, CNIH4, MTOR, which have significant expression change with higher expression in advanced stage compared to normal stage, and proteins, BGN, ITGB2, EPOR, IQGAP1, FN1, RHOB, TSGA10, AR, ZEB1, STAT3, which have significant expression change with lower expression in advanced stage compared to normal stage (Figure 4), are selected as biomarkers in core signaling pathways between middle stage and advanced stage LADC.

The proteins, FGF19, COL3A1, DDR1, CHRNA5, RPL30, CIAPIN1, GSK3B, IGF2BP3, SLC6A11, ERCC1, SUSD4, COL3A1, TERT, SHOX2, YBX1, SP1, ANLN, TP63, which have significant expression change with higher expression in early stage compared to normal stage, and proteins, FGF2, FGFR1, PDGFB, PDGFRB, FKBP5, DAPK3, PSMBB9, ETS1, which have significant expression change with lower expression in early stage compared to normal stage, are selected as biomarkers in core signaling pathways between normal stage and early stage LSCC (Figure 2); the proteins, COL1A1, JAG1, IGF1, IGF1R, COL1A1, TRAF4, SUSD4, PARG, HIF1A, which have significant expression change with higher expression in middle stage compared to normal stage, and proteins, TNS1, ACTA2, CCL21, CCR7, MYLK, PCDP1, ITGB1, TSC2, GATA2, JUN, ETS1, JUNB, which have significant differential expression change with lower expression in middle stage compared to normal stage, are selected as biomarkers in core signaling pathways between early stage and middle stage LSCC (Figure 3); the proteins, CDH3, CHIC2, TUBB3, LIN7C, YBX1, which have significant differential expression change with higher expression in advanced stage compared to normal stage, and proteins, FN1, ITGA8, TGFB1, ERBB4, DDX5, EMR2, MSN, CHD3, AGR3, FOXO3, GATA3, which have significant differential expression change with lower expression in advanced stage compared to normal stage, are selected as biomarkers in core signaling pathways between middle stage and advanced stage LSCC (Figure 4).

To treat early stage, middle stage, and advanced stage of LADC and LSCC from cancer cells back to normal cells, we aim to repress the genes, which have significant differential expression change between normal stage and later stage with higher expression in later stage compared to normal stage, and activate the genes, which have significant differential expression change between normal stage and later stage with lower expression in later stage compared to normal stage without affecting the expression of housekeeping genes for minimizing the side effect. Connectivity Map (CMap) database could provide the level of 14,825 genes under 6,100 different conditions containing 1,327 different compounds (i.e. drugs) and different concentrations of these compounds [185]. The correlation coefficients between the gene expression levels and the concentrations of compounds denote the relationship between compounds and genes. If the correlation coefficient is greater than zero, the gene is said to be up-regulated by applying the compound. If the correlation coefficient is less than zero, the gene is said to be down-regulated by applying the compound. It is noted that we divide the selected biomarkers into two groups. The first group is the pool which we want 
to upregulate (activate). The second group is the pool which we want to downregulate (repress). After applying correlation coefficient between drug concentration and mRNA activity in microarray data of CMap, we then rank 1,327 different compounds (i.e. drugs) based on the satisfaction of our restoration conditions for two groups without affecting gene expression of housekeeping genes by the computational method. Consequently, combined with literature survey to find drugs which have been approved by the Food and Drug Administration (FDA), used in cancer-related therapeutic treatment and reported of having potential anti-cancer properties from the top 20 ranked drugs, we design potential genetic and epigenetic multiple-molecule drug for the therapeutic treatment in early, middle, and advanced stage of LADC and LSCC, respectively.

From core signaling pathways of early stage LADC in Figure 2, we identified a genetic and epigenetic multiple-molecule drug including hydralazine, ketoconazole, and promethazine for potential genetic and epigenetic multiple-molecule drug targets, SLC22A18, TERT, RET, POLR2D, E2F1, PDGFB, EGFL7, S100A10, PDGFRA, TLR4, CCL2, PER1, RB1, TGFB1, SFTPA2, and MYC in core signaling pathways between normal stage and early stage LADC (Table 1). It has been reported that hydralazine, a DNA demethylating agent, was approved by FDA and used in the therapeutic treatment of cancer patient by the combination with a therapeutic dose of valproic acid to mediate the epigenetic modification in tumor cells [186]. Ketoconazole can block the dysregulated cellular metabolism to inhibit the progression of prostate cancer and may be a useful adjunct in treating lung cancer and breast cancer [187, 188]. Besides, promethazine may also be used in treating cancer by modulating energy metabolism in malignant cells [189]. Through the therapeutic treatment using the proposed genetic and epigenetic multiple-molecule drug, the proteins with significant higher expression in the early stage (SLC22A18, TERT, RET, POLR2D, and E2F1) can be repressed, and proteins with significant lower expression in the early stage (PDGFB, EGFL7, S100A10, PDGFRA, TLR4, CCL2, PER1, RB1, TGFB1, SFTPA2, and MYC) can be activated to facilitate the restoration of early stage LADC to normal lung cells.

From core signaling pathways of middle stage LADC in Figure 3, we identified a genetic and epigenetic multiple-molecule drug including betulin, nordihydroguaiaretic acid, and proadifen for potential genetic and epigenetic multiple-molecule drug targets, HP, LTA, APH1A, MMP8, HIF1A, PRDM14, NOTCH1, HGF, ITGA4, VIM, MYH9, ETS1, and ZEB1 in core signaling pathways between early stage and middle stage LADC (Table 2). It has been demonstrated that betulin was effective against tumors, including malignant melanoma, and can cause apoptosis to inhibit the growth of tumor cells [190]. Nordihydroguaiaretic acid can induce apoptosis and inhibit the growth of human lung cancer cells [191]. In addition, nordihydroguaiaretic acid can also inhibit cell migration and tumor metastasis in prostate cancer [192]. Proadifen, an inhibitor of cytochrome P450 monooxygenases, has anti-proliferative properties through influencing metabolic activity, cell number, and cell cycle progression in colon cancer [193]. Through the therapeutic treatment using the proposed genetic and epigenetic multiple-molecule drug, the proteins with significant higher expression in the middle stage (HP, LTA, APH1A, MMP8, HIF1A, and PRDM14) can be repressed, and proteins with significant lower expression in the middle stage (NOTCH1, HGF, ITGA4, VIM, MYH9, ETS1, and ZEB1) can be activated to facilitate the restoration of middle stage LADC to normal lung cells.

From core signaling pathways of advanced stage LADC in Figure 4, we identified a genetic and epigenetic multiple-molecule drug including iloprost, methotrexate, and MK- 886 for potential genetic and epigenetic multiplemolecule drug targets, EGF, CDH2, LOXL2, XRCC4, CNIH4, MTOR, BGN, ITGB2, EPOR, IQGAP1, FN1, RHOB, TSGA10, AR, and STAT3 in core signaling pathways between middle stage and advanced stage LADC (Table 3). It has been reported that the iloprost was studied in randomized phase I trial and can inhibit the transformed growth of NSCLC [194]. Methotrexate is a chemotherapy drugs called anti metabolites and can inhibit the growth of tumor cells, such as lung cancer $[195,196]$. Besides, MK-886 can induce the apoptosis in gastric cancer cells and has the inhibitory ability on cell growth in a dose- and time-dependent manner [197]. Through the therapeutic treatment using the proposed genetic and epigenetic multiple-molecule drug, the proteins with significant higher expression in the advanced stage (EGF, CDH2, LOXL2, XRCC4, CNIH4, and MTOR) can be repressed, and proteins with significant lower expression in the advanced stage (BGN, ITGB2, EPOR, IQGAP1, FN1, RHOB, TSGA10, AR, and STAT3) can be activated to facilitate the restoration of advanced stage LADC to normal lung cells.

From core signaling pathways of early stage LSCC in Figure 2, we identified a genetic and epigenetic multiple-molecule drug including pimozide, mepacrine, and repaglinide for potential genetic and epigenetic multiple-molecule drug targets, COL3A1, DDR1, CHRNA5, RPL30, CIAPIN1, GSK3B, IGF2BP3, SLC6A11, ERCC1, SUSD4, TERT, SHOX2, YBX1, SP1, TP63, FGF2, FGFR1, PDGFB, PDGFRB, FKBP5, DAPK3, and ETS1 in core signaling pathways between normal stage and early stage LSCC (Table 4). It has been shown that pimozide can inhibit prostate cancer cell proliferation through inducing G1 phase cell cycle arrest to decrease the ability of colony formation [198]. Mepacrine, an anti-proliferative agent, can lead to tumor growth inhibition in breast cancer [199]. Besides, repaglinide can inhibit the growth of tumor cells, shch as 
Table 1: Design of genetic and epigenetic multiple drug for the therapeutic treatment of early stage LADC

\begin{tabular}{|c|c|c|}
\hline \multicolumn{3}{|c|}{ Early stage LADC } \\
\hline \multicolumn{3}{|c|}{ Drug target } \\
\hline \multicolumn{3}{|c|}{$\begin{array}{l}\text { SLC22A18, TERT, RET, POLR2D, E2F1, PDGFB, EGFL7, S100A10, PDGFRA, TLR4, CCL2, PER1, RB1, TGFB1, } \\
\text { SFTPA2, MYC }\end{array}$} \\
\hline \multicolumn{3}{|c|}{ Drug molecule } \\
\hline Drug Name & Repressed drug target & Activated drug target \\
\hline Hydralazine & E2F1, SLC22A18, TERT & $\begin{array}{l}\text { CCL2, EGFL7, MYC, PDGFB, PER1, RB1, S100A10, } \\
\text { SFTPA2, TGFB1 }\end{array}$ \\
\hline Ketoconazole & E2F1, POLR2D, RET, SLC22A18, TERT & $\begin{array}{l}\text { CCL2, PDGFB, PDGFRA, S100A10, SFTPA2, TGFB1, } \\
\text { TLR4 }\end{array}$ \\
\hline Promethazine & E2F1, POLR2D, RET, SLC22A18, TERT & $\begin{array}{l}\text { CCL2, PDGFB, PDGFRA, RB1, S100A10, TGFB1, } \\
\text { TLR4 }\end{array}$ \\
\hline
\end{tabular}<smiles>CC(CN1c2ccccc2Sc2ccccc21)N(C)CCN(C)c1ccccc1OCCOCC(C)(C)C</smiles>

Through therapeutic treatment using the proposed genetic and epigenetic multiple-molecule drug, the proteins with significantly higher expression in the early stage (SLC22A18, TERT, RET, POLR2D, and E2F1) can be repressed, and proteins with significant lower expression in the early stage (PDGFB, EGFL7, S100A10, PDGFRA, TLR4, CCL2, PER1, RB1, TGFB1, SFTPA2, and MYC) can be activated to facilitate the restoration of early stage LADC to normal lung cells.

liver hepatocellular carcinoma and cervical cancer [200]. Through the therapeutic treatment using the proposed genetic and epigenetic multiple-molecule drug, the proteins with significant higher expression in the early stage (COL3A1, DDR1, CHRNA5, RPL30, CIAPIN1, GSK3B, IGF2BP3, SLC6A11, ERCC1, SUSD4, TERT, SHOX2, YBX1, SP1, and TP63) can be repressed, and proteins with significant lower expression in the early stage (FGF2, FGFR1, PDGFB, PDGFRB, FKBP5, DAPK3, and ETS1) can be activated to facilitate the restoration of early stage LSCC to normal lung cells.

From core signaling pathways of middle stage LSCC in Figure 3, we identified a genetic and epigenetic multiple-molecule drug including ribavirin, procainamide, and ketoconazole for potential genetic and epigenetic multiple-molecule drug targets, COL1A1, JAG1, IGF1, IGF1R, TRAF4, SUSD4, PARG, TNS1, ACTA2, CCL21, CCR7, MYLK, ITGB1, TSC2, GATA2, JUN, ETS1, and JUNB in core signaling pathways between early stage and middle stage LSCC (Table 5). Ribavirin can impede the oncogenic transformation of cells. It has been reported that ribavirin can cause clinical benefit in poor prognosis acute myeloid leukemia patients in a phase II clinical trial [201]. Procainamide, a drug approved by the FDA, is an inhibitor of DNA methylation and can lead to the reactivation of methylation-silenced genes and demethylation. Previous studies have been reported that procainamide may be potentially used in preventing the development of lung cancer by changing the epigenetic modification [202]. Moreover, ketoconazole can inhibit the progression of prostate cancer and may have potential use in treatment of lung cancer and breast cancer [187, 188]. Through the therapeutic treatment using the proposed genetic and epigenetic multiple-molecule drug, the proteins with significant higher expression in the middle stage (COL1A1, JAG1, IGF1, IGF1R, TRAF4, 
Table 2: Design of genetic and epigenetic multiple drug for the therapeutic treatment of middle stage LADC

\begin{tabular}{ccc}
\hline \multicolumn{2}{c}{ Middle stage LADC } \\
\hline \multicolumn{3}{c}{ Drug target } \\
\hline HP, LTA, APH1A, MMP8, HIF1A, PRDM14, NOTCH1, HGF, ITGA4, VIM, MYH9, ETS1, ZEB1 \\
\hline Drug molecule \\
\hline Drug Name & Repressed drug target & Activated drug target \\
\hline Betulin & APH1A, HIF1A, HP, LTA, MMP8, PRDM14 & HGF, ITGA4, MYH9, NOTCH1, ZEB1 \\
Nordihydroguaiaretic acid & APH1A, HP, MMP8, PRDM14 & ETS1, HGF, ITGA4, MYH9, VIM, ZEB1 \\
Proadifen & APH1A, HIF1A, HP, LTA, MMP8, PRDM14 & HGF, ITGA4, MYH9, ZEB1
\end{tabular}

Chemical structures of multiple-molecule drug

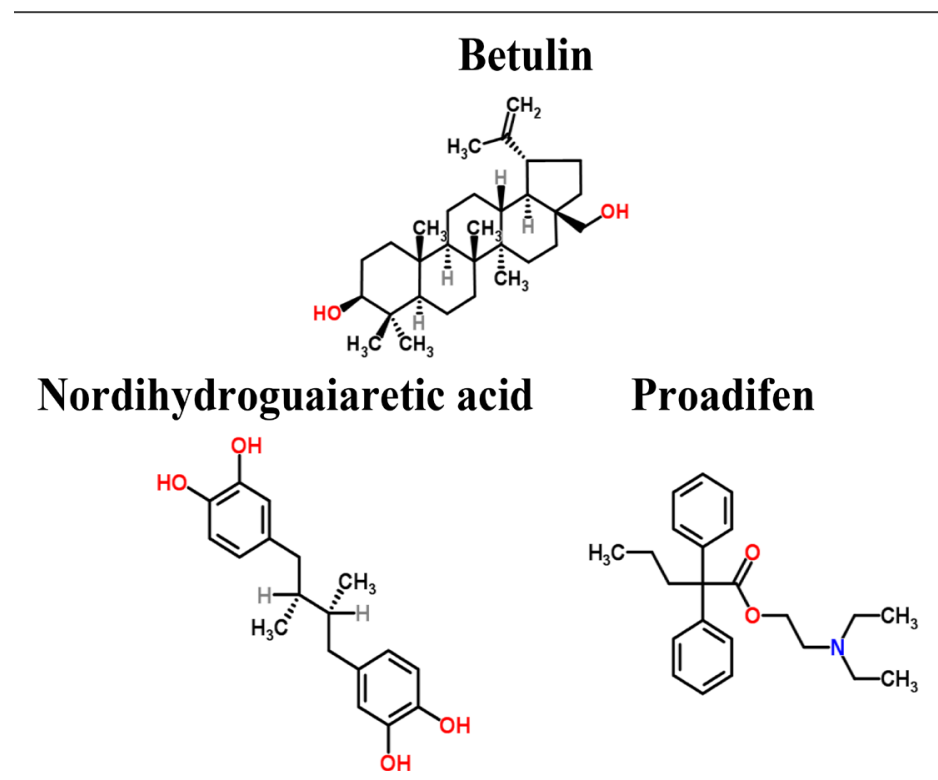

Through therapeutic treatment using the proposed genetic and epigenetic multiple-molecule drug, the proteins with significantly higher expression in the middle stage (HP, LTA, APH1A, MMP8, HIF1A, and PRDM14) can be repressed, and proteins with significant lower expression in the middle stage (NOTCH1, HGF, ITGA4, VIM, MYH9, ETS1 and ZEB1) can be activated to facilitate the restoration of middle stage LADC to normal lung cells.

SUSD4, and PARG) can be repressed, and proteins with significant lower expression in the middle stage (TNS1, ACTA2, CCL21, CCR7, MYLK, ITGB1, TSC2, GATA2, JUN, ETS1, and JUNB) can be activated to facilitate the restoration of middle stage LSCC to normal lung cells.

From core signaling pathways of advanced stage LSCC in Figure 4, we identified a genetic and epigenetic multiple-molecule drug including clomipramine, rolipram, and procainamide for potential genetic and epigenetic multiple-molecule drug targets, CDH3, CHIC2, TUBB3, LIN7C, YBX1, FN1, ITGA8, TGFB1, ERBB4, DDX5, EMR2, MSN, CHD3, FOXO3, and GATA3 in core signaling pathways between middle stage and advanced stage LSCC (Table 6). Clomipramine, an antineoplastic agent, can lead to cancer cell apoptosis. It has been shown that chlorimipramine has positive effects against human leukaemia cells, and human renal cancer cells [203, 204]. Besides, it has been also reported that rolipram can result in a decreased proliferation and an increased apoptosis in malignant glioma cells [205]. Procainamide, an inhibitor of DNA methylation, was approved by the FDA and was potentially used in preventing the development of lung cancer [202]. Through the therapeutic treatment using the proposed genetic and epigenetic multiple-molecule drug, the proteins with significant higher expression in the advanced stage (CDH3, CHIC2, TUBB3, LIN7C, and YBX1) can be repressed, and proteins with significant lower expression in the advanced stage (FN1, ITGA8, TGFB1, ERBB4, DDX5, EMR2, MSN, CHD3, FOXO3, and GATA3) can be activated to facilitate the restoration of advanced stage LSCC to normal lung cells.

Recently, the EGFR-tyrosine kinase inhibitor (TKI) gefitinib (Iressa) drug, which was approved by the FDA, has been used in the treatment of NSCLC [206, 207]. Previous studies reported that using gefitinib in treatment of NSCLC patients have a significant improvement in 
Table 3: Design of genetic and epigenetic multiple drug for the therapeutic treatment of advanced stage LADC

Advanced stage LADC

Drug target

EGF, CDH2, LOXL2, XRCC4, CNIH4, MTOR, BGN, ITGB2, EPOR, IQGAP1, FN1, RHOB, TSGA10, AR, STAT3

Drug molecule

\begin{tabular}{|c|c|c|}
\hline Drug Name & Repressed drug target & Activated drug target \\
\hline Iloprost & $\begin{array}{c}\text { CDH2, CNIH4, EGF, LOXL2, MTOR, } \\
\text { XRCC4 }\end{array}$ & AR, BGN, FN1, RHOB, STAT3, TSGA10 \\
\hline Methotrexate & CDH2, CNIH4, LOXL2, MTOR, XRCC4 & $\begin{array}{c}\text { EPOR, IQGAP1, ITGB2, RHOB, STAT3, } \\
\text { TSGA10 }\end{array}$ \\
\hline MK-886 & $\begin{array}{c}\text { CDH2, CNIH4, EGF, LOXL2, MTOR, } \\
\text { XRCC4 }\end{array}$ & AR, BGN, RHOB, STAT3, TSGA10 \\
\hline
\end{tabular}

Chemical structures of multiple-molecule drug

Iloprost

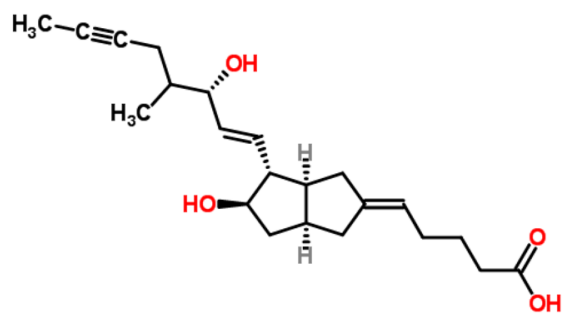

\section{Methotrexate}<smiles>CN(Cc1cnc2nc(N)nc(N)c2n1)c1ccc(C(=O)N[C@@H](CCC(=O)O)C(=O)O)cc1</smiles><smiles>CC(C)c1ccc2c(c1)c(SC(C)(C)C)c(CC(C)(C)C(=O)O)n2Cc1ccc(Cl)cc1</smiles>

Through therapeutic treatment using the proposed genetic and epigenetic multiple-molecule drug, the proteins with significantly higher expression in the advanced stage (EGF, CDH2, LOXL2, XRCC4, CNIH4, and MTOR) can be repressed, and proteins with significant lower expression in the advanced stage (BGN, ITGB2, EPOR, IQGAP1, FN1, RHOB, TSGA10, AR, and STAT3) can be activated to facilitate the restoration of advanced stage LADC to normal lung cells.

the survival of Asian patients [208, 209]. However, our sample data generally are non-Asian patients. Hence we do not consider the drug gefitinib in our designed-genetic and epigenetic multiple-molecular drugs. Moreover, The drug pemetrexed, approved by the FDA, is an antifolate inhibiting multiple enzymes involved in both pyrimidine and purine synthesis. It has been reported that pemetrexed was also used in the treatment of NSCLC patients [210,
211]. However, we did not consider pemetrexed to be our designed-genetic and epigenetic multiple-molecular drugs since it does not include in the 1327 compounds (i.e. drugs) of CMap database.

In conclusion, as shown in Tables 1-6, the six designed genetic and epigenetic multiple-molecular drugs for different stages of LADC and LSCC are given in the following. (1) Hydralazine, ketoconazole, and 
Table 4: Design of genetic and epigenetic multiple drug for the therapeutic treatment of early stage LSCC

Early stage LSCC

Drug target

COL3A1, DDR1, CHRNA5, RPL30, CIAPIN1, GSK3B, IGF2BP3, SLC6A11, ERCC1, SUSD4, TERT, SHOX2, YBX1, SP1, TP63, FGF2, FGFR1, PDGFB, PDGFRB, FKBP5, DAPK3, ETS1

\begin{tabular}{ccc}
\hline & Drug molecule & Repressed drug target \\
\hline Drug Name & CHRNA5, CIAPIN1, COL3A1, DDR1, ERCC1, & Activated drug target \\
\hline \multirow{2}{*}{ Pimozide } & GSK3B, IGF2BP3, RPL30, SHOX2, SLC6A11, & PDGFB \\
& SP1, SUSD4, TERT, TP63, YBX1 & \\
Mepacrine & CHRNA5, CIAPIN1, DDR1, ERCC1, GSK3B, & DAPK3, ETS1, FGF2, FGFR1, PDGFB, \\
& IGF2BP3, RPL30, SUSD4, TERT, YBX1 & PDGRB \\
Repaglinide & CHRNA5, CIAPIN1, COL3A1, DDR1, ERCC1, & FKBP5 \\
& GSK3B, IGF2BP3, RPL30, SHOX2, SLC6A11, & SP1, SUSD4, TERT, TP63, YBX1 \\
\hline
\end{tabular}

Chemical structures of multiple-molecule drug

Pimozide

Mepacrine<smiles>CCN(C)CCCC(C)Nc1c2ccc(Cl)cc2nc2ccc(OC)cc12</smiles><smiles>O=c1[nH]c2ccccc2n1C1CCN(CCCC(c2ccc(F)cc2)c2ccc(F)cc2)CC1</smiles>

Repaglinide<smiles>CCOc1cc(CC(=O)N[C@H](CC(C)C)c2ccccc2N2CCCCC2)ccc1C(=O)O</smiles>

Through therapeutic treatment using the proposed genetic and epigenetic multiple-molecule drug, the proteins with significantly higher expression in the early stage (COL3A1, DDR1, CHRNA5, RPL30, CIAPIN1, GSK3B, IGF2BP3, SLC6A11, ERCC1, SUSD4, TERT, SHOX2, YBX1, SP1, and TP63) can be repressed, and proteins with significant lower expression in the early stage (FGF2, FGFR1, PDGFB, PDGFRB, FKBP5, DAPK3, and ETS1) can be activated to facilitate the restoration of early stage LSCC to normal lung cells.

promethazine are used to repress SLC22A18, TERT, RET, POLR2D, and E2F1 and activate PDGFB, EGFL7, S100A10, and PDGFRA; (2) betulin, nordihydroguaiaretic acid, and proadifen are utilized to repress HP, LTA, APH1A, MMP8, HIF1A, and PRDM14 and activate NOTCH1, HGF, ITGA4, and VIM; (3) iloprost, methotrexate, and MK-886 are designed to repress EGF, $\mathrm{CDH} 2, \mathrm{LOXL} 2, \mathrm{XRCC} 4, \mathrm{CNIH} 4$, and MTOR and activate BGN, ITGB2, EPOR, IQGAP1, FN1, RHOB, TSGA10, $A R$, and STAT3 for the therapeutic treatment of early, middle, and advanced stage of LADC, respectively. Furthermore, (1) pimozide, mepacrine, and repaglinide are used to repress COL3A1, DDR1, CHRNA5, RPL30,
CIAPIN1，GSK3B， IGF2BP3， SLC6A11， ERCC1, SUSD4, TERT, SHOX2, YBX1, SP1, and TP63 and activate FGF2, FGFR1, PDGFB, PDGFRB, FKBP5, DAPK3, and ETS1; (2) ribavirin, procainamide, and ketoconazole are utilized to repress COL1A1, JAG1, IGF1, IGF1R, TRAF4, SUSD4, and PARG and activate TNS1, ACTA2, CCL21, CCR7, MYLK, ITGB1, TSC2, GATA2, JUN, ETS1, and JUNB; (3) clomipramine, rolipram, and procainamide are designed to repress $\mathrm{CDH} 3$, CHIC2, TUBB3, LIN7C, and YBX1 and activate FN1, ITGA8, TGFB1, ERBB4, DDX5, EMR2, MSN, CHD3, FOXO3, and GATA3 for the therapeutic treatment of early, middle, and advanced stage of LSCC, respectively. 
Table 5: Design of genetic and epigenetic multiple drug for the therapeutic treatment of middle stage LSCC

\begin{tabular}{|c|c|c|}
\hline \multicolumn{3}{|c|}{ Middle stage LSCC } \\
\hline \multicolumn{3}{|c|}{ Drug target } \\
\hline \multicolumn{3}{|c|}{$\begin{array}{l}\text { COL1A1, JAG1, IGF1, IGF1R, TRAF4, SUSD4, PARG, TNS1, ACTA2, CCL21, CCR7, MYLK, ITGB1, TSC2, } \\
\text { GATA2, JUN, ETS1, JUNB }\end{array}$} \\
\hline \multicolumn{3}{|c|}{ Drug molecule } \\
\hline Drug Name & Repressed drug target & Activated drug target \\
\hline Ribavirin & COL1A1, IGF1, JAG1, PARG, SUSD4 & $\begin{array}{l}\text { ACTA2, CCL21, CCR7, ETS1, GATA2, ITGB1, } \\
\text { JUN, JUNB, MYLK, TNS1, TSC2 }\end{array}$ \\
\hline Procainamide & IGF1, IGF1R, SUSD4, TRAF4 & $\begin{array}{l}\text { ACTA2, CCL21, CCR7, ETS1, GATA2, ITGB1, } \\
\text { JUN, JUNB, MYLK, TNS1, TSC2 }\end{array}$ \\
\hline Ketoconazole & IGF1, IGF1R, SUSD4, TRAF4 & $\begin{array}{l}\text { ACTA2, CCL21, CCR7, ETS1, GATA2, ITGB1, } \\
\text { JUN, JUNB, MYLK, TNS1, TSC2 }\end{array}$ \\
\hline
\end{tabular}

Chemical structures of multiple-molecule drug

\section{Procainamide}<smiles>CCN(CC)CCNC(=O)c1ccc(N)cc1</smiles>

Ketoconazole<smiles>CC(=O)C1CCC(c2ccc(OCC3COC(c4cccnc4)(c4ccc(Cl)cc4Cl)C3)cc2)CC1</smiles>

Ribavirin<smiles>NC(=O)c1ncn([C@@H]2O[C@H](CO)[C@@H](O)[C@H]2O)n1</smiles>

Through therapeutic treatment using the proposed genetic and epigenetic multiple-molecule drug, the proteins with significantly higher expression in the middle stage (COL1A1, JAG1, IGF1, IGF1R, TRAF4, SUSD4, and PARG) can be repressed, and proteins with significant lower expression in the middle stage (TNS1, ACTA2, CCL21, CCR7, MYLK, ITGB1, TSC2, GATA2, JUN, ETS1, and JUNB) can be activated to facilitate the restoration of middle stage LSCC to normal lung cells.

\section{MATERIALS AND METHODS}

\section{Overview for the construction of genome-wide GENs, core GENs, and core pathways of each progression stage in LADC and LSCC}

To investigate and compare the progression molecular mechanisms between LADC and LSCC from each progression stage (normal stage to early stage, early stage to middle stage, middle stage to advanced stage), we identified genome-wide real GENs and extracted core pathways among the different stages (normal lung cells, early stage, middle stage, and advanced stage) of LADC and LSCC, respectively. The steps for constructing genome-wide real GENs, core GENs, and core pathways between LADC and LSCC from each progression stage are shown in Figure 1. The procedures of constructing core GENs can be divided to four steps: (1) Big databases mining and data preprocessing of gene/miRNA/ lncRNA expression data and DNA methylation data; (2) Constructing candidate GENs by using candidate PPI networks and candidate gene/miRNA/lncRNA regulatory networks (GRNs); (3) Applying system identification method and system order detection scheme with NGS data to obtain real GENs of each stage of LADC and LSCC, respectively; and (4) Using the principal network projection (PNP) method to extract core GENs containing core elements such as core proteins, genes, miRNAs, and lncRNAs from real GENs. By comparing the projection value of each core element in the core GENs, we extracted differential core signaling pathways for each progression stage of LADC and LSCC. Using one- 
Table 6: Design of genetic and epigenetic multiple drugs for the therapeutic treatment of advanced stage LSCC

Advanced stage LSCC

Drug target

CDH3, CHIC2, TUBB3, LIN7C, YBX1, FN1, ITGA8, TGFB1, ERBB4, DDX5, EMR2, MSN, CHD3, FOXO3, GATA3

Drug molecule

\begin{tabular}{ccc}
\hline Drug Name & Repressed drug target & Activated drug target \\
\hline \multirow{2}{*}{ Clomipramine } & $\mathrm{CDH} 3, \mathrm{CHIC2}$, LIN7C, YBX1 & DDX5, EMR2, ERBB4, FN1, FOXO3, GATA3, \\
& ITGA8, TGFB1 \\
Rolipram & CDH3, TUBB3 & DDX5, EMR2, ERBB4, FN1, GATA3, ITGA8, \\
& MSN, TGFB1, CHD3 \\
Procainamide & TUBB3, YBX1 & DDX5, EMR2, ERBB4, FN1, GATA3, ITGA8, \\
\end{tabular}

Chemical structures of multiple-molecule drug

Clomipramine<smiles>CN(C)CCCN1c2ccccc2CCc2ccc(Cl)cc21</smiles>

Rolipram

Procainamide<smiles>COc1ccc(C2CNC(=O)C2)cc1OC1CCCC1</smiles><smiles>CCN(CC)CCNC(=O)c1ccc(N)cc1</smiles>

Through therapeutic treatment using the proposed genetic and epigenetic multiple-molecule drug, the proteins with significantly higher expression in the advanced stage (CDH3, CHIC2, TUBB3, LIN7C, and YBX1) can be repressed, and proteins with significant lower expression in the advanced stage (FN1, ITGA8, TGFB1, ERBB4, DDX5, EMR2, MSN, CHD3, FOXO3, and GATA3) can be activated to facilitate the restoration of advanced stage LSCC to normal lung cells.

way analysis of variance on former and later stage gene expression profile, we also could find out the elements having significant difference with $p$-value $<0.05$ in the core signaling pathways from each progression stage. In the meanwhile, these core signaling pathways are not only with epigenetic modifications but also in KEGG pathway representation giving us insight into the genetic and epigenetic mechanisms of carcinogenic development in LADC and LSCC. Furthermore, we selected potential biomarkers as drugtargets based on our results for designing genetic and epigenetic multiple drugs via mining Cmap drug database for the therapeutic treatment of early stage, middle stage, and advanced stage in LADC and LSCC, respectively.
Big data mining and data preprocessing of NGS data and methylation data for constructing GENs

In this study, we downloaded the genome-wide mRNA, miRNA, and lncRNA NGS data and the DNA methylation profiles from Cancer Brower website (https:// genome-cancer.ucsc.edu/). Two datasets were considered. The one was lung cancer exon expression dataset by RNAseq including genome-wide mRNA, miRNA, and lncRNA expression NGS data. The other one was lung cancer DNA methylation profiles. Lung cancer exon expression dataset was combined from TCGA lung squamous cell carcinoma and lung adenocarcinoma 
datasets. This exon expression profile was measured experimentally using the Illumina HiSeq 2000 RNA Sequencing platform by the University of North Carolina TCGA genome characterization center from 1124 patients (samples). Lung cancer DNA methylation profiles were combined from TCGA lung squamous cell carcinoma and lung adenocarcinoma datasets. This DNA methylation profile was measured experimentally using the Illumina Infinium HumanMethylation450 platform and the beta values were derived at the Johns Hopkins University and University of Southern California TCGA genome characterization center from 907 patients (samples). Because the GENs are constructed by system biology approach based on gene expression level, we averaged each exon expression value, which was derived from the same gene to get the gene expression profile. Based on histopathological types, sample data are subdivided into two major histological subtypes: LADC samples and LSCC samples. According to the seventh edition of the "TNM classification of malignant tumours" published by the UICC, the carcinogenesis of non-small cell lung cancer (NSCLC) can be classified as stage IA, stage IB, stage IIA, stage IIB, stage IIIA, stage IIIB, and stage IV. In this study, we grouped stage IA and stage IB as stage I, stage IIA and stage IIB as stage II, and IIIA and IIIB as stage III, and IV. We further define stage I and stage II as early stage and middle stage, respectively. Due to the fewer samples in stage III and IV, we then grouped stage III and stage IV together as the advanced stage. Hence, the previously mentioned datasets of LADC and LSCC can be divided into four sub-datasets under four conditions, including normal stage (normal lung cells), early stage, middle stage, and advanced stage, respectively. In LADC, there are 276,122 , and 110 tumor samples in early stage, middle stage, and advanced stage, respectively. There are 58 samples in normal cells adjacent to LADC considered to be normal stage of LADC. In LSCC, there are 241, 154, and 93 tumor samples in early stage, middle stage, and advanced stage, respectively. There are 51 samples in normal cells adjacent to LSCC considered to be normal stage of LSCC.

\section{Constructing genome-wide candidate GENs}

Biological processes in different biological conditions between each sample have different significant gene expressions and PPIs. If we only consider the significant genes or proteins in samples, many personal diversity information will be ignored. In order to investigate the biological processes under different lung conditions completely (i.e. normal stage, early stage, middle stage, and advanced stage), we directly consider whole genome information through big databases mining and systems biology approaches. Here, we constructed two candidate genome-wide GENs for LADC and LSCC, respectively. By combining the candidate PPI of LADC with candidate GRN of LADC including regulations between TF/miRNA/lncRNA and their target genes/ miRNAs/lncRNAs, we obtained candidate genome-wide GENs of LADC. The same concept could be used on constructing the candidate genome-wide GEN of LSCC.

The candidate protein-protein interactions (PPIs) were extracted from the databases, including DIP [212], BIND [213], physical interaction part of Biological General Repository for Interaction Datasets (BIOGRID) [214], IntAct [215], and MINT [216]. Besides, the candidate GRNs having transcriptional regulation between transcription factors (TFs) and their target genes extracted from the databases, including Integrated Transcription Factor Platform (ITFP) [217], the Human Transcriptional Regulation Interactions database (HTRIdb) [218], and the candidate TRANScription FACtor database (TRANSFAC) [219]. Moreover, we also obtained the candidate posttranscriptional regulations between miRNAs and target genes from the database TargetScanHuman [220], including all possible targets of miRNAs and regulations between lncRNAs and their target genes from the databases, including CircuitDB [221] and StarBase2.0 [222]. The databases totally contain 4,825,453 candidate interaction pairs between proteins and proteins, 143,707 candidate regulations between TFs and genes, 2,078 candidate regulations between TFs and miRNAs, 302 candidate regulations between TFs and lncRNAs, and 229,620 candidate post-transcriptional regulations between miRNAs and genes, 50 candidate regulations between miRNAs and miRNAs, 700 candidate regulations between miRNAs and lncRNAs, and 374 candidate regulations between lncRNAs and genes (Supplementary Table 3).

\section{Constructing stochastic regression models of candidate PPIN and candidate GRN for candidate GENs in LADC and LSCC}

In previous section, based on big data mining, we have constructed the genome-wide candidate GENs. However, many false positives are included in candidate GENs constructed from numerous databases and experimental datasets, which may contain some plausible information. Therefore, we have to prune these false positives in candidate GENs based on gene/ miRNA/lncRNA expression data and DNA methylation profiles of each lung condition. In this section, we firstly constructed the stochastic regression interactive/regulatory models of human cells to characterize the interactions and regulations in candidate GENs, including PPIs, gene regulations, miRNA regulations, and lncRNA regulations, and epigenetic regulations by DNA methylation.

To identify the real GENs of each lung condition, we applied system identification and system order detection to the interactive/regulatory models of candidate GENs by using gene/miRNA/lncRNA expression data and 
DNA methylation profiles of each lung condition. The significant interactions and regulations out of system order will be considered as false positives in candidate GENs to be pruned to obtain real GENs for LADC and LSCC.

The stochastic regression protein interactive model of the candidate PPIN in candidate GEN, the protein interactions of the $j$ th protein in lung cells for sample $n$ as given by the following:

$$
y_{j}[n]=\sum_{\substack{g=1 \\ j \neq g}}^{J_{j}} \alpha_{j g} y_{g}[n] y_{j}[n]+b_{j}+v_{j}[n], \text { for } j=1, \ldots, J
$$

and $n=1, \ldots, N$

where $y_{j}[n]$ and $y_{g}[n]$ denote the expression level of the $j$-th protein and the $g$-th protein for the $n$-th sample, respectively; $\alpha_{j g}$ is the interaction ability between the $j$-th protein and the $g$-th protein, which is interactive protein of the $j$-th protein; $J_{j}$ represents the number of proteins interacting with the $j$-th protein and $J$ is number of protein with candidate PPIN; $N$ denotes the number of data samples. $b_{j}$ represents the basal level of protein $j . v_{j}[n]$ is the stochastic noise of the $j$-th protein for the sample $n$ due to model uncertainty and data noise. The meaning of protein interactions model equation (1) can be explained that the expression level of the $j$-th protein is related to the interactions with $J_{j}$ other proteins in the candidate PPIN.

The gene regulatory model of candidate GRN, describing the transcriptional regulation of the $i$-th gene of lung cells for sample $n$, is given by the following:

$$
\begin{gathered}
x_{i}[n]=\sum_{\substack{j=1 \\
i \neq j}}^{J_{i}} \beta_{i j} y_{j}[n] M_{i}[n]+\sum_{q=1}^{Q_{i}} \tau_{i q} l_{q}[n] M_{i}[n] \\
-\sum_{p=1}^{P_{i}} \delta_{i p} x_{i}[n] r_{p}[n] M_{i}[n]+k_{i} M_{i}[n]+\varepsilon_{i}[n] \\
\quad \text { for } i=1, \ldots, I \text { and } n=1, \ldots, N
\end{gathered}
$$

where $x_{i}[n], y_{j}[n], l_{q}[\mathrm{n}]$ and $r_{p}[n]$ denote the expression level of the $i$-th target gene, the $j$-th TF, $q$-th lncRNA and $p$-th miRNA for the $n$-th sample, respectively; $\beta_{i j}$ and $\tau_{i q}$ are the transcription regulatory ability of the $j$-th TF and the $q$-th lncRNA on their corresponding binding target gene $i$, respectively. $\delta_{i p}$ indicates the post-transcriptional regulatory ability of the $p$-th miRNA to inhibit the $i$-th target gene $\left(-\delta_{i p} \leq 0\right) . J_{i}, Q_{i}$ and $P_{i}$ represent the number of TFs, IncRNAs and miRNAs binding to the $i$-th target gene, respectively; $I$ and $N$ denotes the number of genes with candidate GRN and the number of data samples, respectively. $k_{i}$ represents the basal level of target gene $i$. $\varepsilon_{i}[n]$ is the stochastic noise of the $i$-th target gene for the sample $n$ due to model uncertainty and data noise. $M_{i}[n]$ denotes the methylation regulation of the $i$-th gene through its effect on the binding affinities of TFs, miRNAs,
lncRNAs, and RNA polymerase on the target gene [27, $223,224]$. The terms $\beta_{i j} y_{j}[n] M_{i}[n], \delta_{i p} x_{i}[n] r_{p}[n] M_{i}[n]$, $\tau_{i q} l[\mathrm{n}] M_{i}[n], k_{i} M_{i}[n]$ denote the effect of methylation on the binding affinities of TFs, miRNAs, lncRNAs, and RNA polymerase to the $i$-th target gene, respectively. The methylation regulation of the $i$-th target gene $M_{i}[n]$ by DNA methylation profile $m_{i}[n]$ can be defined as follows [216]:

$$
M_{i}[n]=\frac{1}{1+\left(\frac{m_{i}[n]}{0.5}\right)^{2}}
$$

where $m_{i}[n]$ indicates the DNA methylation profile of the $i$-th gene for the sample $n$. In equation (3), we can find that the range of effect of DNA methylation on target gene $i M_{i}[n]$ is 1 to 0.2 while DNA methylation profile $m_{i}[n]$ range from 0 to1. From the biological viewpoint, the meaning of equation (3) is that the higher DNA methylation level, the weaker binding affinity between TFs, miRNAs, lncRNAs, and RNA polymerase and their target gene. In contrast, the lower DNA methylation level, the stronger binding affinity between TFs, miRNAs, lncRNAs, and RNA polymerase and their target genes. Besides, the methylation regulation $M_{i}[n]$ in equation (3) still has regulation value $\left(M_{i}[n]=0.2\right)$ while DNA methylation profile is 1 , representing the bindings of TFs, miRNAs, lncRNAs and RNA polymerase to the $i$-th target gene are still exist. The expression level of the $i$-th gene results from transcriptional regulations of TFs and lncRNAs, the post-transcriptional regulations of miRNAs, and expression level of basal level of $i$-th gene with stochastic noise. Besides, the transcriptional regulations of TFs and lncRNAs, the post-transcriptional regulations of miRNAs and expression level of basal level (binding of RNA polymerase) can be affected by DNA methylation.

The miRNA regulatory model of candidate GRN, describing the transcriptional expression of the $p$-th miRNA of lung cells for sample $n$, is given by the following:

$$
\begin{aligned}
& r_{p}[n]=\sum_{\substack{j=1 \\
p \neq j}}^{J_{p}} \lambda_{p j} y_{j}[n] M_{p}[n]- \\
& \sum_{z=1}^{P_{p}} \psi_{p z} r_{p}[n] r_{z}[n] M_{p}[n]+e_{p} M_{p}[n]+\omega_{p}[n],
\end{aligned}
$$

$$
\text { for } p=1, \ldots, P \text { and } n=1, \ldots, N
$$

where $r_{p}[n], y_{j}[n]$, and $r_{z}[\mathrm{n}]$ denote the expression level of the $p$-th miRNA, the $j$-th TF and the $z$-th miRNA for the $n$-th sample, respectively; $\lambda_{p j}$ and $\psi_{p z}$ are the transcription 
regulatory ability of the $j$-th TF on miRNA $p$ and the post-transcriptional regulatory ability of the $p$-th miRNA to inhibit the $p$-th miRNA $\left(-\psi_{p z} \leq 0\right)$. $J_{p}$ represents the number of TF binding to the $p$-th target miRNA; $P$ denotes the number of miRNAs binding to the $p$-th target miRNA and the number of miRNAs with candidate GRN. $P$ denotes the number of miRNA in the candidate GRN. $N$ indicates the number of data samples. $e_{p}$ represents the basal level of target miRNA $p . \omega_{p}[n]$ is the stochastic noise of the $p$-th target miRNA for the sample $n$ due to model uncertainty and data noise. $M_{p}[n]$ denotes methylation regulation of the $p$-th miRNA as shown in equation (3) and $M_{p}[n]$ has the effect on the binding affinities of TFs, miRNAs, and RNA polymerase on the target miRNA. The terms $\lambda_{p j} y_{j}[n] M_{p}[n], \psi_{p z} r_{p}[n] r_{z}[n] M_{p}[n], e_{p} M_{i}[n]$ can express the effect of methylation on the binding affinities of TFs, miRNAs, and RNA polymerase to $p$-th target miRNA, respectively.

Moreover, the lncRNA regulatory model of candidate GRN, describing the transcriptional expression level of the $q$-th lncRNA of lung cells for sample $n$, is given by the following:

$$
\begin{aligned}
& l_{q}[n]=\sum_{\substack{j=1 \\
q \neq j}}^{J_{q}} \gamma_{q j} y_{j}[n] M_{q}[n]- \\
& \sum_{p=1}^{P_{q}} \zeta_{q p} l_{q}[n] r_{p}[n] M_{q}[n]+f_{q} M_{q}[n]+\eta_{q}[n],
\end{aligned}
$$

for $q=1, \ldots, Q$ and $n=1, \ldots, N$

where $l_{q}[n], y_{j}[n]$, and $r_{p}[n]$ denote the expression level of the $q$-th target lncRNA, the $j$-th TF and $p$-th miRNA for the $n$-th sample, respectively; $\gamma_{q j}$ and $\zeta_{q p}$ are the transcription regulatory ability of the $j$-th TF on binding lncRNA $q$ and the post-transcriptional regulatory ability of the $p$-th miRNA to inhibit the $q$-th lncRNA $\left(-\zeta_{q p} \leq 0\right) . J_{q}$ and $P_{q}$ represent the number of TFs and miRNAs binding to the $q$-th lncRNA, respectively; $Q$ and $N$ denotes the number of lncRNAs in candidate GRN and the number of data samples. $f_{q}$ represents the basal level of lncRNA $q . \eta_{g}[n]$ is the stochastic noise of the $q$-th target lncRNA for the sample $n$ due to model uncertainty and data noise. $M_{q}$ $[n]$ denotes methylation regulation of the $q$-th lncRNA as shown in equation (3) and $M_{q}[n]$ has the effect on the binding affinities of TFs, miRNAs, and RNA polymerase on the target lncRNA. The terms $\gamma_{q j} y_{j}[n]$ $M_{q}[n], \quad \zeta_{q p} l_{q}[n] r_{p}[n] M_{q}[n], f_{q} M_{q}[n]$ can express the effect of methylation on the binding affinities of TFs, miRNAs, and RNA polymerase to $q$-th target lncRNA, respectively.

\section{Reversed engineering and principal network projection methods to extract differential core pathways in LADC and LSCC}

After constructing the protein interactive regression model (1) of candidate PPIN and gene/miRNA/lncRNA regulatory models (2), (4), and (5) of candidate GRN, we applied system identification method to do parameter estimation getting the protein interactive parameters $\alpha_{j g}$, $b_{j}$ in protein interactive model and gene/miRNA/lncRNA regulatory parameters $\beta_{i j}, \delta_{i p}, \tau_{i q}, k_{i}, \lambda_{p j}, \psi_{p z}, e_{p}, \gamma_{q j}, \zeta_{q p}, f_{q}$ in gene/miRNA/lncRNA regulatory models by gene/miRNA/ lncRNA expression data and DNA methylation profiles of each stage of LADC and LSCC. Moreover, it is noted that there are many false-positives existing in candidate PPINs and candidate GRNs due to experimental errors. Hence, we used system order detection method to prune false-positives for obtaining real GENs of each stage of LADC and LSCC which are shown in Supplementary Figures 1, 2. The more details of system identification and system order detection methods are in Supplementary Material 1.1. However, it is still too complicated to do analysis on identified GENs. By utilizing principal network projection method in each stage of identified GENs of LADC and LSCC, the core elements could be extracted and kept. In other words, the higher the projection value is, the more important to element contributing to the core GEN. Therefore, comparing the projection value of each element, we constructed core pathways in each stage of LADC and LSCC in respect of KEGG pathway with epigenetic modifications. Based on systems biology approaches mentioned above, we could do further genetic and epigenetic genome-wide analysis on progression molecular mechanisms between LADC and LSCC in the perspective of systematic viewpoint. Detailed information about principal network projection method could be found in Supplementary Material 1.2.

\section{CONCLUSIONS}

In this study, based on mRNA/miRNA/lncRNA and DNA methylation profiles in NGS data, we respectively constructed the GENs for normal stage, early stage, middle stage, and advanced stage of LADC and LSCC to compare the differential genetic and epigenetic progression mechanisms between LADC and LSCC via big data mining, system identification, and system order detection methods. However, the real GENs are still too complex. We used PNP method to obtain core GENs for each stage of LADC and LSCC, respectively. By comparing core GENs among the different stages of LADC and LSCC and with the denotation of KEGG pathways, we further had core pathways in LADC and LSCC between normal lung cells to early stage, early stage to middle stage and middle stage to advanced stage to explore the differential molecular mechanisms between LADC and LSCC. Finally, 
we investigated how the microenvironment changes, epigenetic modifications, miRNA regulations, and lncRNA regulations to affect the differential progression molecular mechanisms between LADC and LSCC. In addition, some significant drug targets are selected from identified network biomarkers based on proteins with significant expression change between later stage and normal stage, we proposed six designed genetic and epigenetic multiple-molecular drugs for therapeutic treatment of early stage, middle stage, and advanced stage LADC and LSCC, respectively. The six designed genetic and epigenetic multiple-molecular drugs are (1) hydralazine, ketoconazole, and promethazine to target SLC22A18, TERT, RET, POLR2D, E2F1, PDGFB, EGFL7, S100A10, PDGFRA, TLR4, CCL2, RB1, TGFB1, PER1, SFTPA2, and MYC for treating early stage LADC; (2) betulin, nordihydroguaiaretic acid, and proadifen to target HP, LTA, APH1A, MMP8, HIF1A, PRDM14, NOTCH1, HGF, ITGA4, VIM, MYH9, ETS1, and ZEB1 for treating middle stage LADC; (3) iloprost, methotrexate, and MK-886 to target EGF, CDH2, LOXL2, XRCC4, CNIH4, MTOR, BGN, ITGB2, EPOR, IQGAP1, FN1, RHOB, TSGA10, AR, and STAT3 for treating advanced stage LADC; (4) pimozide, mepacrine, and repaglinide to target COL3A1, DDR1, CHRNA5, RPL30, CIAPIN1, GSK3B, IGF2BP3, SLC6A11, ERCC1, SUSD4, TERT, SHOX2, YBX1, SP1, TP63, FGF2, FGFR1, PDGFB, PDGFRB, FKBP5, DAPK3, and ETS1 for treating early stage LSCC; (5) ribavirin, procainamide, and ketoconazole to target COL1A1, JAG1, IGF1, IGF1R, TRAF4, SUSD4, PARG, TNS1, ACTA2, CCL21, CCR7, MYLK, ITGB1, TSC2, GATA2, JUN, ETS1, and JUNB for treating middle stage LSCC; (6) clomipramine, rolipram, and rolipram to target CDH3, CHIC2, TUBB3, LIN7C, YBX1, FN1, ITGA8, TGFB1, ERBB4, DDX5, EMR2, MSN, CHD3, FOXO3, and GATA3 for treating advanced stage LSCC, respectively.

\section{Author contributions}

Shan-Ju Yeh, Chien-An Chang, and Cheng-Wei Li designed models, did computational data analysis and contributed to write the manuscript. Lily Hui-Ching Wang helped to provide critical feedback in the final version of the manuscript. Bor-Sen Chen conceived the study and supervised the project.

\section{CONFLICTS OF INTEREST}

The authors declare that there are no conflicts of interest regarding the publication of this paper.

\section{FUNDING}

The work was supported by the Ministry of Science and Technology of Taiwan under grant No. MOST 107-2221-E-007-112-MY3.

\section{REFERENCES}

1. Jemal A, Bray F, Center MM, Ferlay J, Ward E, Forman D. Global cancer statistics. CA Cancer J Clin. 2011; 61:69-90. https://doi.org/10.3322/caac.20107. [PubMed]

2. Siegel R, Naishadham D, Jemal A. Cancer statistics, 2013. CA Cancer J Clin. 2013; 63:11-30. https://doi.org/10.3322/ caac.21166. [PubMed]

3. Ettinger DS, Akerley W, Borghaei H, Chang AC, Cheney RT, Chirieac LR, D'Amico TA, Demmy TL, Govindan R, Grannis FW Jr, Grant SC, Horn L, Jahan TM, et al, and National comprehensive cancer network. Non-small cell lung cancer, version 2.2013. J Natl Compr Canc Netw. 2013; 11:645-53. https://doi.org/10.6004/jnccn.2013.0084. [PubMed]

4. Balgkouranidou I, Liloglou T, Lianidou ES. Lung cancer epigenetics: emerging biomarkers. Biomark Med. 2013; 7:49-58. https://doi.org/10.2217/bmm.12.111. [PubMed]

5. Davidson MR, Gazdar AF, Clarke BE. The pivotal role of pathology in the management of lung cancer. J Thorac Dis. 2013; 5:S463-78. https://doi.org/10.3978/j.issn.20721439.2013.08.43. [PubMed]

6. Shukla S, Khan S, Tollefsbol TO, Meeran SM. Genetics and Epigenetics of Lung Cancer: Mechanisms and Future Perspectives. Curr Cancer Ther Rev. 2013; 9:97-110. https://doi.org/10.2174/15733947113099990002.

7. Chen Z, Fillmore CM, Hammerman PS, Kim CF, Wong KK. Non-small-cell lung cancers: a heterogeneous set of diseases. Nat Rev Cancer. 2014; 14:535-46. https://doi. org $/ 10.1038 / \mathrm{nrc} 3775$. [PubMed]

8. Langer CJ, Besse B, Gualberto A, Brambilla E, Soria JC. The evolving role of histology in the management of advanced non-small-cell lung cancer. J Clin Oncol. 2010; 28:5311-20. https://doi.org/10.1200/JCO.2010.28.8126. [PubMed]

9. Minna JD, Roth JA, Gazdar AF. Focus on lung cancer. Cancer Cell. 2002; 1:49-52. https://doi.org/10.1016/S15356108(02)00027-2. [PubMed]

10. Langevin SM, Kratzke RA, Kelsey KT. Epigenetics of lung cancer. Transl Res. 2015; 165:74-90. https://doi. org/10.1016/j.trsl.2014.03.001. [PubMed]

11. Massion PP, Carbone DP. The molecular basis of lung cancer: molecular abnormalities and therapeutic implications. Respir Res. 2003; 4:12. https://doi. org/10.1186/1465-9921-4-12. [PubMed]

12. Khuder SA. Effect of cigarette smoking on major histological types of lung cancer: a meta-analysis. Lung Cancer. 2001; 31:139-48. https://doi.org/10.1016/S01695002(00)00181-1. [PubMed]

13. Brzeziańska E, Dutkowska A, Antczak A. The significance of epigenetic alterations in lung carcinogenesis. Mol Biol Rep. 2013; 40:309-25. https://doi.org/10.1007/s11033-0122063-4. [PubMed] 
14. Chen QW, Zhu XY, Li YY, Meng ZQ. Epigenetic regulation and cancer (review). Oncol Rep. 2014; 31:523-32. https:// doi.org/10.3892/or.2013.2913. [PubMed]

15. Castro M, Grau L, Puerta P, Gimenez L, Venditti J, Quadrelli S, Sánchez-Carbayo M. Multiplexed methylation profiles of tumor suppressor genes and clinical outcome in lung cancer. J Transl Med. 2010; 8:86. https://doi. org/10.1186/1479-5876-8-86. [PubMed]

16. Hawes SE, Stern JE, Feng Q, Wiens LW, Rasey JS, Lu H, Kiviat NB, Vesselle H. DNA hypermethylation of tumors from non-small cell lung cancer (NSCLC) patients is associated with gender and histologic type. Lung Cancer. 2010; 69:172-79. https://doi.org/10.1016/j. lungcan.2009.11.002. [PubMed]

17. Kwon YJ, Lee SJ, Koh JS, Kim SH, Lee HW, Kang MC, Bae JB, Kim YJ, Park JH. Genome-wide analysis of DNA methylation and the gene expression change in lung cancer. J Thorac Oncol. 2012; 7:20-33. https://doi.org/10.1097/ JTO.0b013e3182307f62. [PubMed]

18. Rouhi A, Mager DL, Humphries RK, Kuchenbauer F. MiRNAs, epigenetics, and cancer. Mamm Genome. 2008; 19:517-25. https://doi.org/10.1007/s00335-008-9133-x. [PubMed]

19. Chen CY, Chen ST, Fuh CS, Juan HF, Huang HC. Coregulation of transcription factors and microRNAs in human transcriptional regulatory network. BMC Bioinformatics. 2011 (Suppl 1); 12:S41. https://doi. org/10.1186/1471-2105-12-S1-S41. [PubMed]

20. Calin GA, Sevignani C, Dumitru CD, Hyslop T, Noch E, Yendamuri S, Shimizu M, Rattan S, Bullrich F, Negrini M, Croce CM. Human microRNA genes are frequently located at fragile sites and genomic regions involved in cancers. Proc Natl Acad Sci U S A. 2004; 101:2999-3004. https:// doi.org/10.1073/pnas.0307323101. [PubMed]

21. Inamura K, Ishikawa Y. MicroRNA In Lung Cancer: Novel Biomarkers and Potential Tools for Treatment. J Clin Med. 2016; 5:E36. https://doi.org/10.3390/jcm5030036. [PubMed]

22. Lin PY, Yu SL, Yang PC. MicroRNA in lung cancer. Br J Cancer. 2010; 103:1144-48. https://doi.org/10.1038/ sj.bjc.6605901. [PubMed]

23. Shi X, Sun M, Liu H, Yao Y, Song Y. Long non-coding RNAs: a new frontier in the study of human diseases. Cancer Lett. 2013; 339:159-66. https://doi.org/10.1016/j. canlet.2013.06.013. [PubMed]

24. Wei MM, Zhou GB. Long Non-coding RNAs and Their Roles in Non-small-cell Lung Cancer. Genomics Proteomics Bioinformatics. 2016; 14:280-88. https://doi. org/10.1016/j.gpb.2016.03.007. [PubMed]

25. Zhang H, Chen Z, Wang X, Huang Z, He Z, Chen Y. Long non-coding RNA: a new player in cancer. J Hematol Oncol. 2013; 6:37. https://doi.org/10.1186/1756-8722-6-37. [PubMed]

26. Kumegawa K, Maruyama R, Yamamoto E, Ashida M, Kitajima H, Tsuyada A, Niinuma T, Kai M, Yamano
HO, Sugai T, Tokino T, Shinomura Y, Imai K, Suzuki H. A genomic screen for long noncoding RNA genes epigenetically silenced by aberrant DNA methylation in colorectal cancer. Sci Rep. 2016; 6:26699. https://doi. org/10.1038/srep26699. [PubMed]

27. Weber M, Hellmann I, Stadler MB, Ramos L, Pääbo S, Rebhan M, Schübeler D. Distribution, silencing potential and evolutionary impact of promoter DNA methylation in the human genome. Nat Genet. 2007; 39:457-66. https:// doi.org/10.1038/ng1990. [PubMed]

28. Li CW, Chang PY, Chen BS. Investigating the mechanism of hepatocellular carcinoma progression by constructing genetic and epigenetic networks using NGS data identification and big database mining method. Oncotarget. 2016; 7:79453-73. https://doi.org/10.18632/ oncotarget.13100. [PubMed]

29. Li CW, Chen BS. Network Biomarkers of Bladder Cancer Based on a Genome-Wide Genetic and Epigenetic Network Derived from Next-Generation Sequencing Data. Dis Markers. 2016; 2016:4149608. https://doi. org/10.1155/2016/4149608. [PubMed]

30. Lin CC, Chen YJ, Chen CY, Oyang YJ, Juan HF, Huang HC. Crosstalk between transcription factors and microRNAs in human protein interaction network. BMC Syst Biol. 2012; 6:18. https://doi.org/10.1186/1752-0509-6-18. [PubMed]

31. Wu CH, Hsu CL, Lu PC, Lin WC, Juan HF, Huang HC. Identification of IncRNA functions in lung cancer based on associated protein-protein interaction modules. Sci Rep. 2016; 6:35939. https://doi.org/10.1038/srep35939. [PubMed]

32. Huang HC, Zheng S, VanBuren V, Zhao Z. Discovering disease-specific biomarker genes for cancer diagnosis and prognosis. Technol Cancer Res Treat. 2010; 9:219-30. https://doi.org/10.1177/153303461000900301. [PubMed]

33. Sirota M, Dudley JT, Kim J, Chiang AP, Morgan AA, Sweet-Cordero A, Sage J, Butte AJ. Discovery and preclinical validation of drug indications using compendia of public gene expression data. Sci Transl Med. 2011; 3:96ra77. https://doi.org/10.1126/scitranslmed.3001318. [PubMed]

34. Chen B, Ma L, Paik H, Sirota M, Wei W, Chua MS, So S, Butte AJ. Reversal of cancer gene expression correlates with drug efficacy and reveals therapeutic targets. Nat Commun. 2017; 8:16022. https://doi.org/10.1038/ncomms16022. [PubMed]

35. Kosti I, Jain N, Aran D, Butte AJ, Sirota M. Cross-tissue Analysis of Gene and Protein Expression in Normal and Cancer Tissues. Sci Rep. 2016; 6:24799. https://doi. org/10.1038/srep24799. [PubMed]

36. Tong AH, Lesage G, Bader GD, Ding H, Xu H, Xin X, Young J, Berriz GF, Brost RL, Chang M, Chen Y, Cheng $\mathrm{X}$, Chua $\mathrm{G}$, et al. Global mapping of the yeast genetic interaction network. Science. 2004; 303:808-13. https:// doi.org/10.1126/science.1091317. [PubMed] 
37. Wasserman S, Faust K. Social Network Analysis: Methods and Applications. 1994. https://doi.org/10.1017/ CBO9780511815478.

38. Shannon P, Markiel A, Ozier O, Baliga NS, Wang JT, Ramage D, Amin N, Schwikowski B, Ideker T. Cytoscape: a software environment for integrated models of biomolecular interaction networks. Genome Res. 2003; 13:2498-504. https://doi.org/10.1101/gr.1239303. [PubMed]

39. Han X, Li F, Fang Z, Gao Y, Li F, Fang R, Yao S, Sun Y, Li L, Zhang W, Ma H, Xiao Q, Ge G, et al. Transdifferentiation of lung adenocarcinoma in mice with Lkb1 deficiency to squamous cell carcinoma. Nat Commun. 2014; 5:3261. https://doi.org/10.1038/ncomms4261. [PubMed]

40. Coussens LM, Werb Z. Inflammation and cancer. Nature. 2002; 420:860-67. https://doi.org/10.1038/nature01322. [PubMed]

41. Lin WW, Karin M. A cytokine-mediated link between innate immunity, inflammation, and cancer. J Clin Invest. 2007; 117:1175-83. https://doi.org/10.1172/JCI31537. [PubMed]

42. Ribeiro R, Araujo A, Lopes C, Medeiros R. Immunoinflammatory mechanisms in lung cancer development: is leptin a mediator? J Thorac Oncol. 2007; 2:105-8. https://doi.org/10.1016/S1556-0864(15)30035-6. [PubMed]

43. Lighthart B. Mini-review of the concentration variations found inthe alfresco atmospheric bacterial populations. Aerobiologia. 2000; 16:7-16. https://doi.org/10.1023/A:1007694618888.

44. Fischer JR, Darjes H, Lahm H, Schindel M, Drings $\mathrm{P}$, Krammer PH. Constitutive secretion of bioactive transforming growth factor beta 1 by small cell lung cancer cell lines. Eur J Cancer. 1994; 30A:2125-9. https://doi. org/10.1016/0959-8049(94)00364-b. [PubMed]

45. Domagała-Kulawik J, Hoser G, Safianowska A, GrubekJaworska H, Chazan R. Elevated TGF-beta1 concentration in bronchoalveolar lavage fluid from patients with primary lung cancer. Arch Immunol Ther Exp (Warsz). 2006; 54:143-47. https://doi.org/10.1007/s00005-006-0016-0. [PubMed]

46. Bennett WP, el-Deiry WS, Rush WL, Guinee DG Jr, Freedman AN, Caporaso NE, Welsh JA, Jones RT, Borkowski A, Travis WD, Fleming MV, Trastek V, Pairolero PC, Tazelaar HD, Midthun D, Jett JR, et al. p21waf1/cip1 and transforming growth factor beta 1 protein expression correlate with survival in non-small cell lung cancer. Clin Cancer Res. 1998; 4:1499-506. https://doi.org/10.1016/ s0169-5002(97)89925-4. [PubMed]

47. Flavell RA, Sanjabi S, Wrzesinski SH, Licona-Limón P. The polarization of immune cells in the tumour environment by TGFbeta. Nat Rev Immunol. 2010; 10:554-67. https://doi. org/10.1038/nri2808. [PubMed]

48. Donin N, Jurianz K, Ziporen L, Schultz S, Kirschfink M, Fishelson Z. Complement resistance of human carcinoma cells depends on membrane regulatory proteins, protein kinases and sialic acid. Clin Exp Immunol. 2003; 131:254-63. https://doi.org/10.1046/j.1365-2249.2003.02066.x. [PubMed]
49. Jurianz K, Ziegler S, Garcia-Schüler H, Kraus S, BohanaKashtan O, Fishelson Z, Kirschfink M. Complement resistance of tumor cells: basal and induced mechanisms. Mol Immunol. 1999; 36:929-39. https://doi.org/10.1016/ S0161-5890(99)00115-7. [PubMed]

50. Verstak B, Nagpal K, Bottomley SP, Golenbock DT, Hertzog PJ, Mansell A. MyD88 adapter-like (Mal)/TIRAP interaction with TRAF6 is critical for TLR2- and TLR4mediated NF-kappaB proinflammatory responses. J Biol Chem. 2009; 284:24192-203. https://doi.org/10.1074/jbc. M109.023044. [PubMed]

51. He W, Liu Q, Wang L, Chen W, Li N, Cao X. TLR4 signaling promotes immune escape of human lung cancer cells by inducing immunosuppressive cytokines and apoptosis resistance. Mol Immunol. 2007; 44:2850-59. https://doi.org/10.1016/j.molimm.2007.01.022. [PubMed]

52. Borron P, McIntosh JC, Korfhagen TR, Whitsett JA, Taylor J, Wright JR. Surfactant-associated protein A inhibits LPSinduced cytokine and nitric oxide production in vivo. Am J Physiol Lung Cell Mol Physiol. 2000; 278:L840-47. https:// doi.org/10.1152/ajplung.2000.278.4.L840. [PubMed]

53. Beckman G, Eklund A, Fröhlander N, Stjernberg N. Haptoglobin groups and lung cancer. Hum Hered. 1986; 36:258-60. https://doi.org/10.1159/000153638. [PubMed]

54. Chang YK, Lai YH, Chu Y, Lee MC, Huang CY, Wu S. Haptoglobin is a serological biomarker for adenocarcinoma lung cancer by using the ProteomeLab PF2D combined with mass spectrometry. Am J Cancer Res. 2016; 6:1828-36. [PubMed]

55. Delfortrie S, Pinte S, Mattot V, Samson C, Villain G, Caetano B, Lauridant-Philippin G, Baranzelli MC, Bonneterre J, Trottein F, Faveeuw C, Soncin F. Egfl7 promotes tumor escape from immunity by repressing endothelial cell activation. Cancer Res. 2011; 71:7176-86. https://doi.org/10.1158/0008-5472.CAN-11-1301. [PubMed]

56. Fitch MJ, Campagnolo L, Kuhnert F, Stuhlmann H. Egfl7, a novel epidermal growth factor-domain gene expressed in endothelial cells. Dev Dyn. 2004; 230:316-24. https://doi. org/10.1002/dvdy.20063. [PubMed]

57. Tang J, Salama R, Gadgeel SM, Sarkar FH, Ahmad A. Erlotinib resistance in lung cancer: current progress and future perspectives. Front Pharmacol. 2013; 4:15. https:// doi.org/10.3389/fphar.2013.00015. [PubMed]

58. Hara H, Yamashita K, Shinada J, Yoshimura H, Kameya T. Clinicopathologic significance of telomerase activity and hTERT mRNA expression in non-small cell lung cancer. Lung Cancer. 2001; 34:219-26. https://doi.org/10.1016/ S0169-5002(01)00244-6. [PubMed]

59. Tan Q, Li F, Wang G, Xia W, Li Z, Niu X, Ji W, Yuan H, Xu Q, Luo Q, Zhang J, Lu S. Identification of FGF19 as a prognostic marker and potential driver gene of lung squamous cell carcinomas in Chinese smoking patients. Oncotarget. 2016; 7:18394-402. https://doi.org/10.18632/ oncotarget.7817. [ PubMed] 
60. Grando SA. Connections of nicotine to cancer. Nat Rev Cancer. 2014; 14:419-29. https://doi.org/10.1038/nrc3725. [PubMed]

61. Sopori M. Effects of cigarette smoke on the immune system. Nat Rev Immunol. 2002; 2:372-77. https://doi.org/10.1038/ nri803. [PubMed]

62. Nouri-Shirazi M, Guinet E. Evidence for the immunosuppressive role of nicotine on human dendritic cell functions. Immunology. 2003; 109:365-73. https://doi. org/10.1046/j.1365-2567.2003.01655.x. [PubMed]

63. Hanahan D, Weinberg RA. Hallmarks of cancer: the next generation. Cell. 2011; 144:646-74. https://doi. org/10.1016/j.cell.2011.02.013. [PubMed]

64. Su B, Zhao W, Shi B, Zhang Z, Yu X, Xie F, Guo Z, Zhang X, Liu J, Shen Q, Wang J, Li X, Zhang Z, Zhou L. Let-7d suppresses growth, metastasis, and tumor macrophage infiltration in renal cell carcinoma by targeting COL3A1 and CCL7. Mol Cancer. 2014; 13:206. https://doi. org/10.1186/1476-4598-13-206. [PubMed]

65. Berger W, Setinek U, Mohr T, Kindas-Mügge I, Vetterlein M, Dekan G, Eckersberger F, Caldas C, Micksche M. Evidence for a role of FGF-2 and FGF receptors in the proliferation of non-small cell lung cancer cells. Int J Cancer. 1999; 83:415-23. https://doi. org/10.1002/(SICI)1097-0215(19991029)83:3<415::AIDIJC19>3.0.CO;2-Y. [ubMed]

66. Godin-Heymann N, Brabetz S, Murillo MM, Saponaro M, Santos CR, Lobley A, East P, Chakravarty P, Matthews N, Kelly G, Jordan S, Castellano E, Downward J. Tumoursuppression function of KLF12 through regulation of anoikis. Oncogene. 2016; 35:3324-34. https://doi. org/10.1038/onc.2015.394. [PubMed]

67. Lomberk G, Urrutia R. The family feud: turning off Sp1 by Sp1-like KLF proteins. Biochem J. 2005; 392:1-11. https:// doi.org/10.1042/BJ20051234. [PubMed]

68. Hsu TI, Wang MC, Chen SY, Yeh YM, Su WC, Chang WC, Hung JJ. Sp1 expression regulates lung tumor progression. Oncogene. 2012; 31:3973-88. https://doi.org/10.1038/ onc.2011.568. [PubMed]

69. Lin PC, Huang HD, Chang CC, Chang YS, Yen JC, Lee CC, Chang WH, Liu TC, Chang JG. Long noncoding RNA TUG1 is downregulated in non-small cell lung cancer and can regulate CELF1 on binding to PRC2. BMC Cancer. 2016; 16:583. https://doi.org/10.1186/s12885-016-2569-6. [PubMed]

70. Zhang E, He X, Yin D, Han L, Qiu M, Xu T, Xia R, Xu L, Yin R, De W. Increased expression of long noncoding RNA TUG1 predicts a poor prognosis of gastric cancer and regulates cell proliferation by epigenetically silencing of p57. Cell Death Dis. 2016; 7:e2109. https://doi.org/10.1038/ cddis.2015.356. [PubMed]

71. Zhang M, Lu W, Huang Y, Shi J, Wu X, Zhang X, Jiang R, Cai Z, Wu S. Downregulation of the long noncoding RNA TUG1 inhibits the proliferation, migration, invasion and promotes apoptosis of renal cell carcinoma. J Mol Histol.
2016; 47:421-28. https://doi.org/10.1007/s10735-0169683-2. [PubMed]

72. Prudkin L, Liu DD, Ozburn NC, Sun M, Behrens C, Tang X, Brown KC, Bekele BN, Moran C, Wistuba II. Epithelial-to-mesenchymal transition in the development and progression of adenocarcinoma and squamous cell carcinoma of the lung. Mod Pathol. 2009; 22:668-78. https://doi.org/10.1038/modpathol.2009.19. [PubMed]

73. Baumgart A, Seidl S, Vlachou P, Michel L, Mitova N, Schatz N, Specht K, Koch I, Schuster T, Grundler R, Kremer M, Fend F, Siveke JT, et al. ADAM17 regulates epidermal growth factor receptor expression through the activation of Notch1 in non-small cell lung cancer. Cancer Res. 2010; 70:5368-78. https://doi.org/10.1158/0008-5472. CAN-09-3763. [PubMed]

74. Donnem T, Andersen S, Al-Shibli K, Al-Saad S, Busund LT, Bremnes RM. Prognostic impact of Notch ligands and receptors in nonsmall cell lung cancer: coexpression of Notch-1 and vascular endothelial growth factor-A predicts poor survival. Cancer. 2010; 116:5676-85. https://doi. org/10.1002/cncr.25551. [PubMed]

75. Hsu YL, Wu CY, Hung JY, Lin YS, Huang MS, Kuo PL. Galectin-1 promotes lung cancer tumor metastasis by potentiating integrin $\alpha 6 \beta 4$ and Notch1/Jagged 2 signaling pathway. Carcinogenesis. 2013; 34:1370-81. https://doi. org/10.1093/carcin/bgt040. [PubMed]

76. Jiang X, Zhou JH, Deng ZH, Qu XH, Jiang HY, Liu Y. [Expression and significance of Notch1, Jagged1 and VEGF in human non-small cell lung cancer]. [Article in Chinese]. Zhong Nan Da Xue Xue Bao Yi Xue Ban. 2007; 32:1031-6. https://doi.org/10.1016/j.cellbi.2008.01.171. [PubMed]

77. Yang Y, Ahn YH, Gibbons DL, Zang Y, Lin W, Thilaganathan N, Alvarez CA, Moreira DC, Creighton CJ, Gregory PA, Goodall GJ, Kurie JM. The Notch ligand Jagged 2 promotes lung adenocarcinoma metastasis through a miR-200-dependent pathway in mice. J Clin Invest. 2011; 121:1373-85. https://doi.org/10.1172/JCI42579. [PubMed]

78. Yuan X, Wu H, Xu H, Han N, Chu Q, Yu S, Chen Y, Wu K. Meta-analysis reveals the correlation of Notch signaling with non-small cell lung cancer progression and prognosis. Sci Rep. 2015; 5:10338. https://doi.org/10.1038/srep10338. [PubMed]

79. Zhou L, Wu S, Yu L, Gong X, Song W, Cheng Z. [Expression of CD133 and Notch1 in non-small cell lung cancer and the clinicopathological significance]. [Article in Chinese]. Nan Fang Yi Ke Da Xue Xue Bao. 2015; 35:196201. [ $\underline{\text { PubMed] }}$

80. Chen Y, De Marco MA, Graziani I, Gazdar AF, Strack PR, Miele L, Bocchetta M. Oxygen concentration determines the biological effects of NOTCH-1 signaling in adenocarcinoma of the lung. Cancer Res. 2007; 67:7954-59. https://doi. org/10.1158/0008-5472.CAN-07-1229. [PubMed]

81. Oh MK, Park HJ, Kim NH, Park SJ, Park IY, Kim IS. Hypoxia-inducible factor-1alpha enhances haptoglobin gene expression by improving binding of STAT3 to the 
promoter. J Biol Chem. 2011; 286:8857-65. https://doi. org/10.1074/jbc.M110.150557. [PubMed]

82. Ding F, Bao C, Tian Y, Xiao H, Wang M, Xie X, Hu F, Mei J. USP22 promotes NSCLC tumorigenesis via MDMX up-regulation and subsequent p53 inhibition. Int J Mol Sci. 2014; 16:307-20. https://doi.org/10.3390/ijms16010307. [PubMed]

83. Wang X. p53 regulation: teamwork between RING domains of Mdm2 and MdmX. Cell Cycle. 2011; 10:4225-29. https://doi.org/10.4161/cc.10.24.18662. [PubMed]

84. Jin Y, Zeng SX, Sun XX, Lee H, Blattner C, Xiao Z, Lu H. MDMX promotes proteasomal turnover of p21 at G1 and early S phases independently of, but in cooperation with, MDM2. Mol Cell Biol. 2008; 28:1218-29. https://doi. org/10.1128/MCB.01198-07. [PubMed]

85. Pan Y, Chen J. MDM2 promotes ubiquitination and degradation of MDMX. Mol Cell Biol. 2003; 23:511321. https://doi.org/10.1128/MCB.23.15.5113-5121.2003. [PubMed]

86. Slabáková E, Kharaishvili G, Smějová M, Pernicová Z, Suchánková T, Remšík J, Lerch S, Straková N, Bouchal J, Král M, Culig Z, Kozubík A, Souček K. Opposite regulation of MDM2 and MDMX expression in acquisition of mesenchymal phenotype in benign and cancer cells. Oncotarget. 2015; 6:36156-71. https://doi.org/10.18632/ oncotarget.5392. [PubMed]

87. Takeyama Y, Sato M, Horio M, Hase T, Yoshida K, Yokoyama T, Nakashima H, Hashimoto N, Sekido Y, Gazdar AF, Minna JD, Kondo M, Hasegawa Y. Knockdown of ZEB1, a master epithelial-to-mesenchymal transition (EMT) gene, suppresses anchorage-independent cell growth of lung cancer cells. Cancer Lett. 2010; 296:216-24. https:// doi.org/10.1016/j.canlet.2010.04.008. [PubMed]

88. Larsen JE, Nathan V, Osborne JK, Farrow RK, Deb D, Sullivan JP, Dospoy PD, Augustyn A, Hight SK, Sato M, Girard L, Behrens C, Wistuba II, et al. ZEB1 drives epithelial-to-mesenchymal transition in lung cancer. J Clin Invest. 2016; 126:3219-35. https://doi.org/10.1172/ JCI76725. [PubMed]

89. Pietras A, von Stedingk K, Lindgren D, Påhlman S, Axelson H. JAG2 induction in hypoxic tumor cells alters Notch signaling and enhances endothelial cell tube formation. Mol Cancer Res. 2011; 9:626-36. https://doi.org/10.1158/15417786.MCR-10-0508. [PubMed]

90. Harris AL. Hypoxia - a key regulatory factor in tumour growth. Nat Rev Cancer. 2002; 2:38-47. https://doi. org/10.1038/nrc704. [PubMed]

91. Pouysségur J, Dayan F, Mazure NM. Hypoxia signalling in cancer and approaches to enforce tumour regression. Nature. 2006; 441:437-43. https://doi.org/10.1038/ nature04871. [PubMed]

92. Semenza GL. Hypoxia-inducible factors in physiology and medicine. Cell. 2012; 148:399-408. https://doi. org/10.1016/j.cell.2012.01.021. [PubMed]
93. Giatromanolaki A, Koukourakis MI, Sivridis E, Turley H, Talks K, Pezzella F, Gatter KC, Harris AL. Relation of hypoxia inducible factor 1 alpha and 2 alpha in operable non-small cell lung cancer to angiogenic/molecular profile of tumours and survival. Br J Cancer. 2001; 85:881-90. https://doi.org/10.1054/bjoc.2001.2018. [PubMed]

94. Lee CH, Lee MK, Kang CD, Kim YD, Park DY, Kim JY, Sol MY, Suh KS. Differential expression of hypoxia inducible factor-1 alpha and tumor cell proliferation between squamous cell carcinomas and adenocarcinomas among operable non-small cell lung carcinomas. J Korean Med Sci. 2003; 18:196-203. https://doi.org/10.3346/ jkms.2003.18.2.196. [PubMed]

95. Zhang H, Zhang Z, Xu Y, Xing L, Liu J, Li J, Tan Q. The expression of hypoxia inducible factor 1-alpha in lung cancer and its correlation with P53 and VEGF. J Huazhong Univ Sci Technolog Med Sci. 2004; 24:124-7. https://doi. org $/ 10.1007 / \mathrm{bf02885408}$. [PubMed]

96. Karetsi E, Ioannou MG, Kerenidi T, Minas M, Molyvdas PA, Gourgoulianis KI, Paraskeva E. Differential expression of hypoxia-inducible factor $1 \alpha$ in non-small cell lung cancer and small cell lung cancer. Clinics (Sao Paulo). 2012; 67:1373-78. https://doi.org/10.6061/clinics/2012(12)05. [PubMed]

97. Erler JT, Bennewith KL, Nicolau M, Dornhöfer N, Kong C, Le QT, Chi JT, Jeffrey SS, Giaccia AJ. Lysyl oxidase is essential for hypoxia-induced metastasis. Nature. 2006; 440:1222-26. https://doi.org/10.1038/nature04695. [PubMed]

98. Ping W, Jiang WY, Chen WS, Sun W, Fu XN. Expression and significance of hypoxia inducible factor- $1 \alpha$ and lysyl oxidase in non-small cell lung cancer. Asian Pac J Cancer Prev. 2013; 14:3613-18. https://doi.org/10.7314/ APJCP.2013.14.6.3613. [PubMed]

99. Wilgus ML, Borczuk AC, Stoopler M, Ginsburg M, Gorenstein L, Sonett JR, Powell CA. Lysyl oxidase: a lung adenocarcinoma biomarker of invasion and survival. Cancer. 2011; 117:2186-91. https://doi.org/10.1002/ cncr.25768. [PubMed]

100. Mendez MG, Kojima S, Goldman RD. Vimentin induces changes in cell shape, motility, and adhesion during the epithelial to mesenchymal transition. FASEB J. 2010; 24:1838-51. https://doi.org/10.1096/fj.09-151639. [PubMed]

101. Thiery JP. Epithelial-mesenchymal transitions in tumour progression. Nat Rev Cancer. 2002; 2:442-54. https://doi. org/10.1038/nrc822. [PubMed]

102. Jiang J, Lv X, Fan L, Huang G, Zhan Y, Wang M, Lu H. MicroRNA-27b suppresses growth and invasion of NSCLC cells by targeting Sp1. Tumour Biol. 2014; 35:10019-23. https://doi.org/10.1007/s13277-014-2294-1. [PubMed]

103. Wan L, Zhang L, Fan K, Wang J. MiR-27b targets LIMK1 to inhibit growth and invasion of NSCLC cells. Mol Cell Biochem. 2014; 390:85-91. https://doi.org/10.1007/s11010013-1959-1. [PubMed] 
104. Goto Y, Kojima S, Nishikawa R, Enokida H, Chiyomaru T, Kinoshita T, Nakagawa M, Naya Y, Ichikawa T, Seki N. The microRNA-23b/27b/24-1 cluster is a disease progression marker and tumor suppressor in prostate cancer. Oncotarget. 2014; 5:7748-59. https://doi.org/10.18632/oncotarget.2294. [PubMed]

105. Veliceasa D, Biyashev D, Qin G, Misener S, Mackie AR, Kishore R, Volpert OV. Therapeutic manipulation of angiogenesis with miR-27b. Vasc Cell. 2015; 7:6. https:// doi.org/10.1186/s13221-015-0031-1. [PubMed]

106. Betapudi V, Licate LS, Egelhoff TT. Distinct roles of nonmuscle myosin II isoforms in the regulation of MDA-MB-231 breast cancer cell spreading and migration. Cancer Res. 2006; 66:4725-33. https://doi. org/10.1158/0008-5472.CAN-05-4236. [PubMed]

107. Derycke L, Stove C, Vercoutter-Edouart AS, De Wever O, Dollé L, Colpaert N, Depypere H, Michalski JC, Bracke $\mathrm{M}$. The role of non-muscle myosin IIA in aggregation and invasion of human MCF-7 breast cancer cells. Int J Dev Biol. 2011; 55:835-40. https://doi.org/10.1387/ ijdb.113336ld. [PubMed]

108. Katono K, Sato Y, Jiang SX, Kobayashi M, Nagashio R, Ryuge S, Fukuda E, Goshima N, Satoh Y, Saegusa M, Masuda N. Prognostic significance of MYH9 expression in resected non-small cell lung cancer. PLoS One. 2015; 10:e0121460. https://doi.org/10.1371/journal. pone.0121460. [ [PubMed]

109. Ramaswamy S, Ross KN, Lander ES, Golub TR. A molecular signature of metastasis in primary solid tumors. Nat Genet. 2003; 33:49-54. https://doi.org/10.1038/ng1060. [PubMed]

110. Oleksiewicz U, Liloglou T, Tasopoulou KM, Daskoulidou N, Gosney JR, Field JK, Xinarianos G. COL1A1, PRPF40A, and UCP2 correlate with hypoxia markers in non-small cell lung cancer. J Cancer Res Clin Oncol. 2017; 143:1133-41. https://doi.org/10.1007/s00432-017-2381-y. [PubMed]

111. Nilsson K, Landberg G. Subcellular localization, modification and protein complex formation of the cdk-inhibitor p16 in Rb-functional and $\mathrm{Rb}$-inactivated tumor cells. Int J Cancer. 2006; 118:1120-25. https://doi. org/10.1002/ijc.21466. [PubMed]

112. Souza-Rodrígues E, Estanyol JM, Friedrich-Heineken E, Olmedo E, Vera J, Canela N, Brun S, Agell N, Hübscher U, Bachs O, Jaumot M. Proteomic analysis of p16ink4abinding proteins. Proteomics. 2007; 7:4102-11. https://doi. org/10.1002/pmic.200700133. [PubMed]

113. Zhang Q, Tang X, Zhang ZF, Velikina R, Shi S, Le AD. Nicotine induces hypoxia-inducible factor-1alpha expression in human lung cancer cells via nicotinic acetylcholine receptor-mediated signaling pathways. Clin Cancer Res. 2007; 13:4686-94. https://doi. org/10.1158/1078-0432.CCR-06-2898. [PubMed]

114. Acosta JC, O’Loghlen A, Banito A, Guijarro MV, Augert A, Raguz S, Fumagalli M, Da Costa M, Brown C, Popov
N, Takatsu Y, Melamed J, d'Adda di Fagagna F, et al. Chemokine signaling via the CXCR2 receptor reinforces senescence. Cell. 2008; 133:1006-18. https://doi. org/10.1016/j.cell.2008.03.038. [PubMed]

115. Behrens P, Rothe M, Wellmann A, Krischler J, Wernert N. The Ets-1 transcription factor is up-regulated together with MMP 1 and MMP 9 in the stroma of pre-invasive breast cancer. J Pathol. 2001; 194:43-50. https://doi.org/10.1002/ path.844. [PubMed]

116. Saeki H, Kuwano H, Kawaguchi H, Ohno S, Sugimachi $\mathrm{K}$. Expression of ets-1 transcription factor is correlated with penetrating tumor progression in patients with squamous cell carcinoma of the esophagus. Cancer.

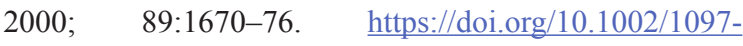
0142(20001015)89:8<1670::AID-CNCR4>3.0.CO;2-J.

[PubMed]

117. Logan SK, Garabedian MJ, Campbell CE, Werb Z. Synergistic transcriptional activation of the tissue inhibitor of metalloproteinases-1 promoter via functional interaction of AP-1 and Ets-1 transcription factors. J Biol Chem. 1996; 271:774-82. https://doi.org/10.1074/jbc.271.2.774. [PubMed]

118. Pollak MN, Schernhammer ES, Hankinson SE. Insulinlike growth factors and neoplasia. Nat Rev Cancer. 2004; 4:505-18. https://doi.org/10.1038/nrc1387. [PubMed]

119. Chen HX, Sharon E. IGF-1R as an anti-cancer target-trials and tribulations. Chin J Cancer. 2013; 32:242-52. https:// doi.org/10.5732/cjc.012.10263. [PubMed]

120. Sachdev D, Yee D. Disrupting insulin-like growth factor signaling as a potential cancer therapy. Mol Cancer Ther. 2007; 6:1-12. https://doi.org/10.1158/1535-7163.MCT-060080. [PubMed]

121. Nurwidya F, Takahashi F, Kobayashi I, Murakami A, Kato M, Minakata K, Nara T, Hashimoto M, Yagishita S, Baskoro H, Hidayat M, Shimada N, Takahashi K. Treatment with insulin-like growth factor 1 receptor inhibitor reverses hypoxia-induced epithelial-mesenchymal transition in non-small cell lung cancer. Biochem Biophys Res Commun. 2014; 455:332-38. https://doi.org/10.1016/j. bbrc.2014.11.014. [PubMed]

122. Björndahl M, Cao R, Nissen LJ, Clasper S, Johnson LA, Xue Y, Zhou Z, Jackson D, Hansen AJ, Cao Y. Insulin-like growth factors 1 and 2 induce lymphangiogenesis in vivo. Proc Natl Acad Sci U S A. 2005; 102:15593-98. https://doi. org/10.1073/pnas.0507865102. [PubMed]

123. Zhang Q, Sun L, Yin L, Ming J, Zhang S, Luo W, Qiu X. CCL19/CCR7 upregulates heparanase via specificity protein-1 (Sp1) to promote invasion of cell in lung cancer. Tumour Biol. 2013; 34:2703-08. https://doi.org/10.1007/ s13277-013-0822-z. [PubMed]

124. Tutunea-Fatan E, Majumder M, Xin X, Lala PK. The role of CCL21/CCR7 chemokine axis in breast cancer-induced lymphangiogenesis. Mol Cancer. 2015; 14:35. https://doi. org/10.1186/s12943-015-0306-4. [PubMed] 
125. Lee HW, Park YM, Lee SJ, Cho HJ, Kim DH, Lee JI, Kang MS, Seol HJ, Shim YM, Nam DH, Kim HH, Joo KM. Alpha-smooth muscle actin (ACTA2) is required for metastatic potential of human lung adenocarcinoma. Clin Cancer Res. 2013; 19:5879-89. https://doi. org/10.1158/1078-0432.CCR-13-1181. [PubMed]

126. Rhodes DR, Yu J, Shanker K, Deshpande N, Varambally R, Ghosh D, Barrette T, Pandey A, Chinnaiyan AM. Large-scale meta-analysis of cancer microarray data identifies common transcriptional profiles of neoplastic transformation and progression. Proc Natl Acad Sci U S A. 2004; 101:9309-14. https://doi.org/10.1073/ pnas.0401994101. [PubMed]

127. Wang XM, Li J, Yan MX, Liu L, Jia DS, Geng Q, Lin $\mathrm{HC}, \mathrm{He} \mathrm{XH}, \mathrm{Li} J J$, Yao M. Integrative analyses identify osteopontin, LAMB3 and ITGB1 as critical pro-metastatic genes for lung cancer. PLoS One. 2013; 8:e55714. https:// doi.org/10.1371/journal.pone.0055714. [PubMed]

128. Zheng W, Jiang C, Li R. Integrin and gene network analysis reveals that ITGA5 and ITGB1 are prognostic in non-smallcell lung cancer. Onco Targets Ther. 2016; 9:2317-27. https://doi.org/10.2147/OTT.S91796. [PubMed]

129. Birsoy K, Possemato R, Lorbeer FK, Bayraktar EC, Thiru P, Yucel B, Wang T, Chen WW, Clish CB, Sabatini DM. Metabolic determinants of cancer cell sensitivity to glucose limitation and biguanides. Nature. 2014; 508:108-12. https://doi.org/10.1038/nature13110. [PubMed]

130. Popper HH. Progression and metastasis of lung cancer. Cancer Metastasis Rev. 2016; 35:75-91. https://doi. org/10.1007/s10555-016-9618-0. [PubMed]

131. Kasapović J, Pejić S, Stojiljković V, Todorović A, Radošević-Jelić L, Saičić ZS, Pajović SB. Antioxidant status and lipid peroxidation in the blood of breast cancer patients of different ages after chemotherapy with 5-fluorouracil, doxorubicin and cyclophosphamide. Clin Biochem. 2010; 43:1287-93. https://doi.org/10.1016/j. clinbiochem.2010.08.009. [PubMed]

132. Martinez-Outschoorn UE, Lin Z, Trimmer C, Flomenberg N, Wang C, Pavlides S, Pestell RG, Howell A, Sotgia F, Lisanti MP. Cancer cells metabolically "fertilize" the tumor microenvironment with hydrogen peroxide, driving the Warburg effect: implications for PET imaging of human tumors. Cell Cycle. 2011; 10:2504-20. https://doi. org/10.4161/cc.10.15.16585. [PubMed]

133. Martinez-Outschoorn UE, Whitaker-Menezes D, Lin Z, Flomenberg N, Howell A, Pestell RG, Lisanti MP, Sotgia F. Cytokine production and inflammation drive autophagy in the tumor microenvironment: role of stromal caveolin-1 as a key regulator. Cell Cycle. 2011; 10:1784-93. https://doi. org/10.4161/cc.10.11.15674. [PubMed]

134. Whitaker-Menezes D, Martinez-Outschoorn UE, Lin Z, Ertel A, Flomenberg N, Witkiewicz AK, Birbe RC, Howell A, Pavlides S, Gandara R, Pestell RG, Sotgia F, Philp NJ, Lisanti MP. Evidence for a stromal-epithelial "lactate shuttle" in human tumors: MCT4 is a marker of oxidative stress in cancer-associated fibroblasts. Cell Cycle. 2011; 10:1772-83. https://doi.org/10.4161/cc.10.11.15659. [PubMed]

135. Witkiewicz AK, Kline J, Queenan M, Brody JR, Tsirigos A, Bilal E, Pavlides S, Ertel A, Sotgia F, Lisanti MP. Molecular profiling of a lethal tumor microenvironment, as defined by stromal caveolin-1 status in breast cancers. Cell Cycle. 2011; 10:1794-809. https://doi.org/10.4161/cc.10.11.15675. [PubMed]

136. Lisanti MP, Martinez-Outschoorn UE, Lin Z, Pavlides S, Whitaker-Menezes D, Pestell RG, Howell A, Sotgia F. Hydrogen peroxide fuels aging, inflammation, cancer metabolism and metastasis: the seed and soil also needs "fertilizer". Cell Cycle. 2011; 10:2440-49. https://doi. org/10.4161/cc.10.15.16870. [PubMed]

137. Ndele JK, Yoshioka K, Fisher JW. Hydrogen peroxide in the regulation of erythropoietin (Epo) gene expression in hepatocellular carcinoma cells. East Afr Med J. 1996; 73:143-46. [ubMed]

138. Tóvári J, Pirker R, Tímár J, Ostoros G, Kovács G, Döme B. Erythropoietin in cancer: an update. Curr Mol Med. 2008; 8:481-91. https://doi.org/10.2174/156652408785747979. [PubMed]

139. Kinsella MG, Bressler SL, Wight TN. The regulated synthesis of versican, decorin, and biglycan: extracellular matrix proteoglycans that influence cellular phenotype. Crit Rev Eukaryot Gene Expr. 2004; 14:203-34. https:// doi.org/10.1615/CritRevEukaryotGeneExpr.v14.i3.40. [PubMed]

140. Maishi N, Ohba Y, Akiyama K, Ohga N, Hamada J, NagaoKitamoto H, Alam MT, Yamamoto K, Kawamoto T, Inoue N, Taketomi A, Shindoh M, Hida Y, Hida K. Tumour endothelial cells in high metastatic tumours promote metastasis via epigenetic dysregulation of biglycan. Sci Rep. 2016; 6:28039. https://doi.org/10.1038/srep28039. [PubMed]

141. Wadhwa S, Embree MC, Bi Y, Young MF. Regulation, regulatory activities, and function of biglycan. Crit Rev Eukaryot Gene Expr. 2004; 14:301-15. https:// doi.org/10.1615/CritRevEukaryotGeneExpr.v14.i4.50. [PubMed]

142. Yamamoto K, Ohga N, Hida Y, Maishi N, Kawamoto T, Kitayama K, Akiyama K, Osawa T, Kondoh M, Matsuda $\mathrm{K}$, Onodera Y, Fujie M, Kaga K, et al. Biglycan is a specific marker and an autocrine angiogenic factor of tumour endothelial cells. Br J Cancer. 2012; 106:1214-23. https:// doi.org/10.1038/bjc.2012.59. [PubMed]

143. Wang X, Chen Z. MicroRNA-19a functions as an oncogenic microRNA in non-small cell lung cancer by targeting the suppressor of cytokine signaling 1 and mediating STAT3 activation. Int J Mol Med. 2015; 35:839-46. https://doi. org/10.3892/ijmm.2015.2071. [PubMed]

144. Labernadie A, Kato T, Brugués A, Serra-Picamal X, Derzsi S, Arwert E, Weston A, González-Tarragó V, Elosegui-Artola A, Albertazzi L, Alcaraz J, Roca-Cusachs P, Sahai E, Trepat X. A mechanically active heterotypic 
E-cadherin/N-cadherin adhesion enables fibroblasts to drive cancer cell invasion. Nat Cell Biol. 2017; 19:224-37. https://doi.org/10.1038/ncb3478. [PubMed]

145. Yamauchi M, Yoshino I, Yamaguchi R, Shimamura T, Nagasaki M, Imoto S, Niida A, Koizumi F, Kohno T, Yokota J, Miyano S, Gotoh N. N-cadherin expression is a potential survival mechanism of gefitinib-resistant lung cancer cells. Am J Cancer Res. 2011; 1:823-33. [PubMed]

146. Zhang X, Liu G, Kang Y, Dong Z, Qian Q, Ma X. $\mathrm{N}$-cadherin expression is associated with acquisition of EMT phenotype and with enhanced invasion in erlotinibresistant lung cancer cell lines. PLoS One. 2013; 8:e57692. https://doi.org/10.1371/journal.pone.0057692. [PubMed]

147. Leithner K. PEPCK in cancer cell starvation. Oncoscience. 2015; 2:805-06. https://doi.org/10.18632/oncoscience.252. [PubMed]

148. Ridley AJ. Rho proteins and cancer. Breast Cancer Res Treat. 2004; 84:13-19. https://doi.org/10.1023/ B:BREA.0000018423.47497.c6. [PubMed]

149. Sahai E, Marshall CJ. RHO-GTPases and cancer. Nat Rev Cancer. 2002; 2:133-42. https://doi.org/10.1038/nrc725. [PubMed]

150. Mazieres J, Antonia T, Daste G, Muro-Cacho C, Berchery D, Tillement V, Pradines A, Sebti S, Favre G. Loss of RhoB expression in human lung cancer progression. Clin Cancer Res. 2004; 10:2742-50. https://doi.org/10.1158/1078-0432. CCR-03-0149. [PubMed]

151. Sato N, Fukui T, Taniguchi T, Yokoyama T, Kondo M, Nagasaka T, Goto Y, Gao W, Ueda Y, Yokoi K, Minna JD, Osada H, Kondo Y, Sekido Y. RhoB is frequently downregulated in non-small-cell lung cancer and resides in the 2 p24 homozygous deletion region of a lung cancer cell line. Int J Cancer. 2007; 120:543-51. https://doi. org/10.1002/ijc.22328. [PubMed]

152. Wang S, Yan-Neale Y, Fischer D, Zeremski M, Cai R, Zhu J, Asselbergs F, Hampton G, Cohen D. Histone deacetylase 1 represses the small GTPase RhoB expression in human nonsmall lung carcinoma cell line. Oncogene. 2003; 22:6204 13. https://doi.org/10.1038/sj.onc.1206653. [PubMed]

153. Wang Z, Luo Z, Zhou L, Li X, Jiang T, Fu E. DDX5 promotes proliferation and tumorigenesis of non-small-cell lung cancer cells by activating $\beta$-catenin signaling pathway. Cancer Sci. 2015; 106:1303-12. https://doi.org/10.1111/ cas.12755. [PubMed]

154. Lai AY, Wade PA. Cancer biology and NuRD: a multifaceted chromatin remodelling complex. Nat Rev Cancer. 2011; 11:588-96. https://doi.org/10.1038/nrc3091. [PubMed]

155. King ER, Tung CS, Tsang YT, Zu Z, Lok GT, Deavers MT, Malpica A, Wolf JK, Lu KH, Birrer MJ, Mok SC, Gershenson DM, Wong KK. The anterior gradient homolog 3 (AGR3) gene is associated with differentiation and survival in ovarian cancer. Am J Surg Pathol. 2011; 35:904-12. https://doi.org/10.1097/PAS.0b013e318212ae22. [PubMed]
156. Obacz J, Takacova M, Brychtova V, Dobes P, Pastorekova S, Vojtesek B, Hrstka R. The role of AGR2 and AGR3 in cancer: similar but not identical. Eur J Cell Biol. 2015; 94:139-47. https://doi.org/10.1016/j.ejcb.2015.01.002. [PubMed]

157. Emerling BM, Weinberg F, Liu JL, Mak TW, Chandel NS. PTEN regulates p300-dependent hypoxia-inducible factor 1 transcriptional activity through Forkhead transcription factor 3a (FOXO3a). Proc Natl Acad Sci U S A. 2008; 105:2622-27. https://doi.org/10.1073/pnas.0706790105. [PubMed]

158. Furukawa-Hibi Y, Yoshida-Araki K, Ohta T, Ikeda K, Motoyama N. FOXO forkhead transcription factors induce $\mathrm{G}(2)-\mathrm{M}$ checkpoint in response to oxidative stress. J Biol Chem. 2002; 277:26729-32. https://doi.org/10.1074/jbc. C200256200. [PubMed]

159. Kops GJ, Dansen TB, Polderman PE, Saarloos I, Wirtz KW, Coffer PJ, Huang TT, Bos JL, Medema RH, Burgering BM. Forkhead transcription factor $\mathrm{FOXO} 3$ a protects quiescent cells from oxidative stress. Nature. 2002; 419:316-21. https://doi.org/10.1038/nature01036. [PubMed]

160. Tran H, Brunet A, Grenier JM, Datta SR, Fornace AJ Jr, DiStefano PS, Chiang LW, Greenberg ME. DNA repair pathway stimulated by the forkhead transcription factor FOXO3a through the Gadd45 protein. Science. 2002; 296:530 34. https://doi.org/10.1126/science.1068712. [PubMed]

161. Blake DC Jr, Mikse OR, Freeman WM, Herzog CR. FOXO3a elicits a pro-apoptotic transcription program and cellular response to human lung carcinogen nicotinederived nitrosaminoketone (NNK). Lung Cancer. 2010; 67:37-47. https://doi.org/10.1016/j.lungcan.2009.03.013. [PubMed]

162. Huang H, Tindall DJ. Dynamic FoxO transcription factors. J Cell Sci. 2007; 120:2479-87. https://doi.org/10.1242/ jes.001222. [PubMed]

163. Nakamura N, Ramaswamy S, Vazquez F, Signoretti S, Loda M, Sellers WR. Forkhead transcription factors are critical effectors of cell death and cell cycle arrest downstream of PTEN. Mol Cell Biol. 2000; 20:8969-82. https://doi. org/10.1128/MCB.20.23.8969-8982.2000. [PubMed]

164. Skovseth DK, Veuger MJ, Sorensen DR, De Angelis PM, Haraldsen G. Endostatin dramatically inhibits endothelial cell migration, vascular morphogenesis, and perivascular cell recruitment in vivo. Blood. 2005; 105:1044-51. https:// doi.org/10.1182/blood-2004-03-1164. [PubMed]

165. Zhang Y, Yu LK, Lu GJ, Xia N, Xie HY, Hu W, Hao KK, $\mathrm{Xu} \mathrm{CH}$, Qian Q. Prognostic values of VEGF and endostatin with malignant pleural effusions in patients with lung cancer. Asian Pac J Cancer Prev. 2014; 15:8435-40. https:// doi.org/10.7314/APJCP.2014.15.19.8435. [PubMed]

166. Wang Q, Xu D, Han C, Tu M, Du Q, Zhang J, Zhu Y, Xu L. Overexpression of serine/threonine-protein kinase-1 in pancreatic cancer tissue: Serine/threonine-protein kinase-1 knockdown increases the chemosensitivity of pancreatic 
cancer cells. Mol Med Rep. 2015; 12:475-81. https://doi. org/10.3892/mmr.2015.3434. [PubMed]

167. Xu GF, O'Connell P, Viskochil D, Cawthon R, Robertson M, Culver M, Dunn D, Stevens J, Gesteland R, White R, Weiss R. The neurofibromatosis type 1 gene encodes a protein related to GAP. Cell. 1990; 62:599-608. https://doi. org/10.1016/0092-8674(90)90024-9. [PubMed]

168. Cawthon RM, Weiss R, Xu GF, Viskochil D, Culver M, Stevens J, Robertson M, Dunn D, Gesteland R, O'Connell $\mathrm{P}$, White R. A major segment of the neurofibromatosis type 1 gene: cDNA sequence, genomic structure, and point mutations. Cell. 1990; 62:193-201. https://doi. org/10.1016/0092-8674(90)90253-B. [PubMed]

169. Martin GA, Viskochil D, Bollag G, McCabe PC, Crosier WJ, Haubruck H, Conroy L, Clark R, O'Connell P, Cawthon RM, et al. The GAP-related domain of the neurofibromatosis type 1 gene product interacts with ras p21. Cell. 1990; 63:843-49. https://doi.org/10.1016/00928674(90)90150-D. [PubMed]

170. Le LQ, Parada LF. Tumor microenvironment and neurofibromatosis type I: connecting the GAPs. Oncogene. 2007; 26:4609-16. https://doi.org/10.1038/sj.onc.1210261. [PubMed]

171. Ozawa T, Araki N, Yunoue S, Tokuo H, Feng L, Patrakitkomjorn S, Hara T, Ichikawa Y, Matsumoto K, Fujii K, Saya H. The neurofibromatosis type 1 gene product neurofibromin enhances cell motility by regulating actin filament dynamics via the Rho-ROCK-LIMK2-cofilin pathway. J Biol Chem. 2005; 280:39524-33. https://doi. org/10.1074/jbc.M503707200. [PubMed]

172. Kato Y, Kaneko M, Sata M, Fujita N, Tsuruo T, Osawa M. Enhanced expression of Aggrus (T1alpha/podoplanin), a platelet-aggregation-inducing factor in lung squamous cell carcinoma. Tumour Biol. 2005; 26:195-200. https://doi. org/10.1159/000086952. [PubMed]

173. Wicki A, Lehembre F, Wick N, Hantusch B, Kerjaschki D, Christofori G. Tumor invasion in the absence of epithelialmesenchymal transition: podoplanin-mediated remodeling of the actin cytoskeleton. Cancer Cell. 2006; 9:261-72. https://doi.org/10.1016/j.ccr.2006.03.010. [PubMed]

174. Cueni LN, Hegyi I, Shin JW, Albinger-Hegyi A, Gruber S, Kunstfeld R, Moch H, Detmar M. Tumor lymphangiogenesis and metastasis to lymph nodes induced by cancer cell expression of podoplanin. Am J Pathol. 2010; 177:1004-16. https://doi.org/10.2353/ajpath.2010.090703. [PubMed]

175. Arenas MI, Romo E, Royuela M, Fraile B, Paniagua R. E-, N- and P-cadherin, and alpha-, beta- and gammacatenin protein expression in normal, hyperplastic and carcinomatous human prostate. Histochem J. 2000; 32:65967. https://doi.org/10.1023/A:1004111331752. [PubMed]

176. Kümper S, Ridley AJ. p120ctn and P-cadherin but not E-cadherin regulate cell motility and invasion of DU145 prostate cancer cells. PLoS One. 2010; 5:e11801. https:// doi.org/10.1371/journal.pone.0011801. [PubMed]
177. Paredes J, Albergaria A, Oliveira JT, Jeronimo C, Milanezi F, Schmitt FC. P-cadherin overexpression is an indicator of clinical outcome in invasive breast carcinomas and is associated with $\mathrm{CDH} 3$ promoter hypomethylation. Clin Cancer Res. 2005; 11:5869-77. https://doi. org/10.1158/1078-0432.CCR-05-0059. [PubMed]

178. Sun L, Hu H, Peng L, Zhou Z, Zhao X, Pan J, Sun L, Yang Z, Ran Y. P-cadherin promotes liver metastasis and is associated with poor prognosis in colon cancer. Am J Pathol. 2011; 179:380-90. https://doi.org/10.1016/j. ajpath.2011.03.046. [PubMed]

179. Yi S, Yang ZL, Miao X, Zou Q, Li J, Liang L, Zeng G, Chen S. N-cadherin and P-cadherin are biomarkers for invasion, metastasis, and poor prognosis of gallbladder carcinomas. Pathol Res Pract. 2014; 210:363-68. https:// doi.org/10.1016/j.prp.2014.01.014. [PubMed]

180. Ng MR, Besser A, Danuser G, Brugge JS. Substrate stiffness regulates cadherin-dependent collective migration through myosin-II contractility. J Cell Biol. 2012; 199:54563. https://doi.org/10.1083/jcb.201207148. [PubMed]

181. Nguyen-Ngoc KV, Cheung KJ, Brenot A, Shamir ER, Gray RS, Hines WC, Yaswen P, Werb Z, Ewald AJ. ECM microenvironment regulates collective migration and local dissemination in normal and malignant mammary epithelium. Proc Natl Acad Sci U S A. 2012; 109:E2595604. https://doi.org/10.1073/pnas.1212834109. [PubMed]

182. Plutoni C, Bazellieres E, Le Borgne-Rochet M, Comunale F, Brugues A, Séveno M, Planchon D, Thuault S, Morin $\mathrm{N}$, Bodin S, Trepat X, Gauthier-Rouvière C. P-cadherin promotes collective cell migration via a Cdc42-mediated increase in mechanical forces. J Cell Biol. 2016; 212:199217. https://doi.org/10.1083/jcb.201505105. [PubMed]

183. Davies JQ, Lin HH, Stacey M, Yona S, Chang GW, Gordon S, Hamann J, Campo L, Han C, Chan P, Fox SB. Leukocyte adhesion-GPCR EMR2 is aberrantly expressed in human breast carcinomas and is associated with patient survival. Oncol Rep. 2011; 25:619-27. https://doi.org/10.3892/ or.2010.1117. [PubMed]

184. Safaee M, Ivan ME, Oh MC, Oh T, Sayegh ET, Kaur G, Sun MZ, Bloch O, Parsa AT. The role of epidermal growth factor-like module containing mucin-like hormone receptor 2 in human cancers. Oncol Rev. 2014; 8:242. https://doi. org/10.4081/oncol.2014.242. [PubMed]

185. Lamb J, Crawford ED, Peck D, Modell JW, Blat IC, Wrobel MJ, Lerner J, Brunet JP, Subramanian A, Ross KN, Reich M, Hieronymus H, Wei G, et al. The Connectivity Map: using gene-expression signatures to connect small molecules, genes, and disease. Science. 2006; 313:1929-35. https://doi.org/10.1126/ science.1132939. [PubMed]

186. Bauman J, Shaheen M, Verschraegen CF, Belinsky SA, Houman Fekrazad M, Lee FC, Rabinowitz I, Ravindranathan M, Jones DV Jr. A Phase I Protocol of Hydralazine and Valproic Acid in Advanced, Previously Treated Solid Cancers. Transl Oncol. 2014 Apr 17. 
https://doi.org/10.1016/j.tranon.2014.03.001. [Epub ahead of print]. [PubMed]

187. Trachtenberg J. Ketoconazole therapy in advanced prostatic cancer. J Urol. 1987; 137:959. https://doi.org/10.1016/ S0022-5347(17)44309-6. [PubMed]

188. Vanuytsel L, Ang KK, Vantongelen K, Drochmans A, Baert L, van der Schueren E. Ketoconazole therapy for advanced prostatic cancer: feasibility and treatment results. J Urol. 1987; 137:905-08. https://doi.org/10.1016/S00225347(17)44291-1. [PubMed]

189. Jones GR. Successful cancer therapy with promethazine: the rationale. Med Hypotheses. 1996; 46:25-29. https://doi. org/10.1016/S0306-9877(96)90231-5. [PubMed]

190. Drąg-Zalesińska M, Drąg M, Poręba M, Borska S, Kulbacka J, Saczko J. Anticancer properties of ester derivatives of betulin in human metastatic melanoma cells (Me-45). Cancer Cell Int. 2017; 17:4. https://doi.org/10.1186/s12935016-0369-3. [PubMed]

191. Seufferlein T, Seckl MJ, Schwarz E, Beil M, v Wichert G, Baust H, Lührs H, Schmid RM, Adler G. Mechanisms of nordihydroguaiaretic acid-induced growth inhibition and apoptosis in human cancer cells. Br J Cancer. 2002; 86:118896. https://doi.org/10.1038/sj.bjc.6600186. [PubMed]

192. Li X, Fan S, Pan X, Xiaokaiti Y, Duan J, Shi Y, Pan Y, Tie L, Wang X, Li Y, Li X. Nordihydroguaiaretic acid impairs prostate cancer cell migration and tumor metastasis by suppressing neuropilin 1. Oncotarget. 2016; 7:86225-38. https://doi.org/10.18632/oncotarget.13368. [PubMed]

193. Jendzelovsky R, Koval J, Mikes J, Papcova Z, Plsikova J, Fedorocko P. Inhibition of GSK-3beta reverses the proapoptotic effect of proadifen (SKF-525A) in HT-29 colon adenocarcinoma cells. Toxicol In Vitro. 2012; 26:775-82. https://doi.org/10.1016/j.tiv.2012.05.014. [PubMed]

194. Tennis MA, Van Scoyk M, Heasley LE, Vandervest K, Weiser-Evans M, Freeman S, Keith RL, Simpson P, Nemenoff RA, Winn RA. Prostacyclin inhibits non-small cell lung cancer growth by a frizzled 9-dependent pathway that is blocked by secreted frizzled-related protein 1 . Neoplasia. 2010; 12:244-53. https://doi.org/10.1593/ neo.91690. [PubMed]

195. Chen YH, Tsai CY, Huang PY, Chang MY, Cheng PC, Chou $\mathrm{CH}$, Chen DH, Wang CR, Shiau AL, Wu CL. Methotrexate conjugated to gold nanoparticles inhibits tumor growth in a syngeneic lung tumor model. Mol Pharm. 2007; 4:713-22. https://doi.org/10.1021/mp060132k. [PubMed]

196. Djerassi I, Rominger CJ, Kim JS, Turchi J, Suvansri U, Hughes D. Phase I study of high doses of methotrexate with citrovorum factor in patients with lung cancer. Cancer. 1972; 30:22-30. https:// doi.org/10.1002/1097-0142(197207)30:1<22::AIDCNCR2820300105>3.0.CO;2-B. [PubMed]

197. Fan XM, Tu SP, Lam SK, Wang WP, Wu J, Wong WM, Yuen MF, Lin MC, Kung HF, Wong BC. Five-lipoxygenaseactivating protein inhibitor MK-886 induces apoptosis in gastric cancer through upregulation of p27kip1 and bax. J Gastroenterol Hepatol. 2004; 19:31-37. https://doi. org/10.1111/j.1440-1746.2004.03194.x. [PubMed]

198. Zhou W, Chen MK, Yu HT, Zhong ZH, Cai N, Chen GZ, Zhang P, Chen JJ. The antipsychotic drug pimozide inhibits cell growth in prostate cancer through suppression of STAT3 activation. Int J Oncol. 2016; 48:322-28. https:// doi.org/10.3892/ijo.2015.3229. [PubMed]

199. Rossi T, Coppi A, Bruni E, Ruberto A, Giudice S, Baggio G. Mepacrine antagonises tumour cell growth induced by natural polyamines. Anticancer Res. 2008; 28:2765-68. [PubMed]

200. El Sharkawi FZ, El Shemy HA, Khaled HM. Possible anticancer activity of rosuvastatine, doxazosin, repaglinide and oxcarbazepin. Asian Pac J Cancer Prev. 2014; 15:199203. https://doi.org/10.7314/APJCP.2014.15.1.199. [PubMed]

201. Borden KL, Culjkovic-Kraljacic B. Ribavirin as an anticancer therapy: acute myeloid leukemia and beyond? Leuk Lymphoma. 2010; 51:1805-15. https://doi.org/10.3109/104 28194.2010.496506. [PubMed]

202. Gao Z, Xu Z, Hung MS, Lin YC, Wang T, Gong M, Zhi $X$, Jablons DM, You L. Procaine and procainamide inhibit the Wnt canonical pathway by promoter demethylation of WIF-1 in lung cancer cells. Oncol Rep. 2009; 22:1479-84. https://doi.org/10.3892/or_00000590. [PubMed]

203. Bilir A, Erguven M, Oktem G, Ozdemir A, Uslu A, Aktas E, Bonavida B. Potentiation of cytotoxicity by combination of imatinib and chlorimipramine in glioma. Int J Oncol. 2008; 32:829-39. https://doi.org/10.3892/ijo.32.4.829. [PubMed]

204. Daley E, Wilkie D, Loesch A, Hargreaves IP, Kendall DA, Pilkington GJ, Bates TE. Chlorimipramine: a novel anticancer agent with a mitochondrial target. Biochem Biophys Res Commun. 2005; 328:623-32. https://doi. org/10.1016/j.bbrc.2005.01.028. [PubMed]

205. Chen TC, Wadsten P, Su S, Rawlinson N, Hofman FM, Hill $\mathrm{CK}$, Schönthal AH. The type IV phosphodiesterase inhibitor rolipram induces expression of the cell cycle inhibitors p21(Cip1) and p27(Kip1), resulting in growth inhibition, increased differentiation, and subsequent apoptosis of malignant A-172 glioma cells. Cancer Biol Ther. 2002; 1:268-76. https://doi.org/10.4161/cbt.80. [PubMed]

206. Armour AA, Watkins CL. The challenge of targeting EGFR: experience with gefitinib in nonsmall cell lung cancer. Eur Respir Rev. 2010; 19:186-96. https://doi. org/10.1183/09059180.00005110. [PubMed]

207. Nurwidya F, Takahashi F, Takahashi K. Gefitinib in the treatment of nonsmall cell lung cancer with activating epidermal growth factor receptor mutation. J Nat Sci Biol Med. 2016; 7:119-23. https://doi.org/10.4103/09769668.184695. [PubMed]

208. Chang A, Parikh P, Thongprasert S, Tan EH, Perng RP, Ganzon D, Yang CH, Tsao CJ, Watkins C, Botwood N, Thatcher N. Gefitinib (IRESSA) in patients of Asian origin with refractory advanced non-small cell lung cancer: subset 
analysis from the ISEL study. J Thorac Oncol. 2006; 1:84755. https://doi.org/10.1097/01243894-200610000-00014. [PubMed]

209. Jiang H. Overview of gefitinib in non-small cell lung cancer: an Asian perspective. Jpn J Clin Oncol. 2009; 39:137-50. https://doi.org/10.1093/jico/hyn139. [PubMed]

210. Scagliotti G, Hanna N, Fossella F, Sugarman K, Blatter J, Peterson P, Simms L, Shepherd FA. The differential efficacy of pemetrexed according to NSCLC histology: a review of two Phase III studies. Oncologist. 2009; 14:253-63. https:// doi.org/10.1634/theoncologist.2008-0232. [PubMed]

211. Tomasini P, Barlesi F, Mascaux C, Greillier L. Pemetrexed for advanced stage nonsquamous non-small cell lung cancer: latest evidence about its extended use and outcomes. Ther Adv Med Oncol. 2016; 8:198-208. https://doi. org/10.1177/1758834016644155. [PubMed]

212. Salwinski L, Miller CS, Smith AJ, Pettit FK, Bowie JU, Eisenberg D. The Database of Interacting Proteins: 2004 update. Nucleic Acids Res. 2004; 32:D449-51. https://doi. org/10.1093/nar/gkh086. [PubMed]

213. Bader GD, Betel D, Hogue CW. BIND: the Biomolecular Interaction Network Database. Nucleic Acids Res. 2003; 31:248-50. https://doi.org/10.1093/nar/gkg056. [PubMed]

214. Chatr-Aryamontri A, Breitkreutz BJ, Oughtred R, Boucher L, Heinicke S, Chen D, Stark C, Breitkreutz A, Kolas N, O'Donnell L, Reguly T, Nixon J, Ramage L, et al. The BioGRID interaction database: 2015 update. Nucleic Acids Res. 2015; 43:D470-78. https://doi.org/10.1093/nar/ gku1204. [PubMed]

215. Orchard S, Ammari M, Aranda B, Breuza L, Briganti L, Broackes-Carter F, Campbell NH, Chavali G, Chen C, delToro N, Duesbury M, Dumousseau M, Galeota E, et al. The MIntAct project-IntAct as a common curation platform for 11 molecular interaction databases. Nucleic Acids Res. 2014; 42:D358-63. https://doi.org/10.1093/nar/gkt1115. [PubMed]

216. Licata L, Briganti L, Peluso D, Perfetto L, Iannuccelli M, Galeota E, Sacco F, Palma A, Nardozza AP, Santonico E, Castagnoli L, Cesareni G. MINT, the molecular interaction database: 2012 update. Nucleic Acids Res. 2012; 40:D85761. https://doi.org/10.1093/nar/gkr930. [PubMed]
217. Zheng G, Tu K, Yang Q, Xiong Y, Wei C, Xie L, Zhu Y, Li Y. ITFP: an integrated platform of mammalian transcription factors. Bioinformatics. 2008; 24:2416-17. https://doi. org/10.1093/bioinformatics/btn439. [PubMed]

218. Bovolenta LA, Acencio ML, Lemke N. HTRIdb: an open-access database for experimentally verified human transcriptional regulation interactions. BMC Genomics. 2012; 13:405. https://doi.org/10.1186/1471-2164-13-405. [PubMed]

219. Wingender E. The TRANSFAC project as an example of framework technology that supports the analysis of genomic regulation. Brief Bioinform. 2008; 9:326-32. https://doi. org/10.1093/bib/bbn016. [PubMed]

220. Agarwal V, Bell GW, Nam JW, Bartel DP. Predicting effective microRNA target sites in mammalian mRNAs. Elife. 2015; 4:4. https://doi.org/10.7554/eLife.05005. [PubMed]

221. Friard O, Re A, Taverna D, De Bortoli M, Corá D. CircuitsDB: a database of mixed microRNA/transcription factor feed-forward regulatory circuits in human and mouse. BMC Bioinformatics. 2010; 11:435. https://doi. org/10.1186/1471-2105-11-435. [PubMed]

222. Li JH, Liu S, Zhou H, Qu LH, Yang JH. starBase v2.0: decoding miRNA-ceRNA, miRNA-ncRNA and proteinRNA interaction networks from large-scale CLIP-Seq data. Nucleic Acids Res. 2014; 42:D92-97. https://doi. org/10.1093/nar/gkt1248. [PubMed]

223. Medvedeva YA, Khamis AM, Kulakovskiy IV, Ba-Alawi W, Bhuyan MS, Kawaji H, Lassmann T, Harbers M, Forrest AR, Bajic VB, and FANTOM consortium. Effects of cytosine methylation on transcription factor binding sites. BMC Genomics. 2014; 15:119. https://doi. org/10.1186/1471-2164-15-119. [PubMed]

224. Bandres E, Agirre X, Bitarte N, Ramirez N, Zarate R, Roman-Gomez J, Prosper F, Garcia-Foncillas J. Epigenetic regulation of microRNA expression in colorectal cancer. Int J Cancer. 2009; 125:2737-43. https://doi.org/10.1002/ ijc.24638. [PubMed] 